Prepared for the U.S. Army Corps of Engineers, Portland District

under an Interagency Agreement with the U.S. Department of Energy

Contract DE-AC05-76RL01830

\title{
JSATS Decoder Software Manual
}

\section{FINAL REPORT}

AE Flory

BL LaMarche

MA Weiland

May 2013

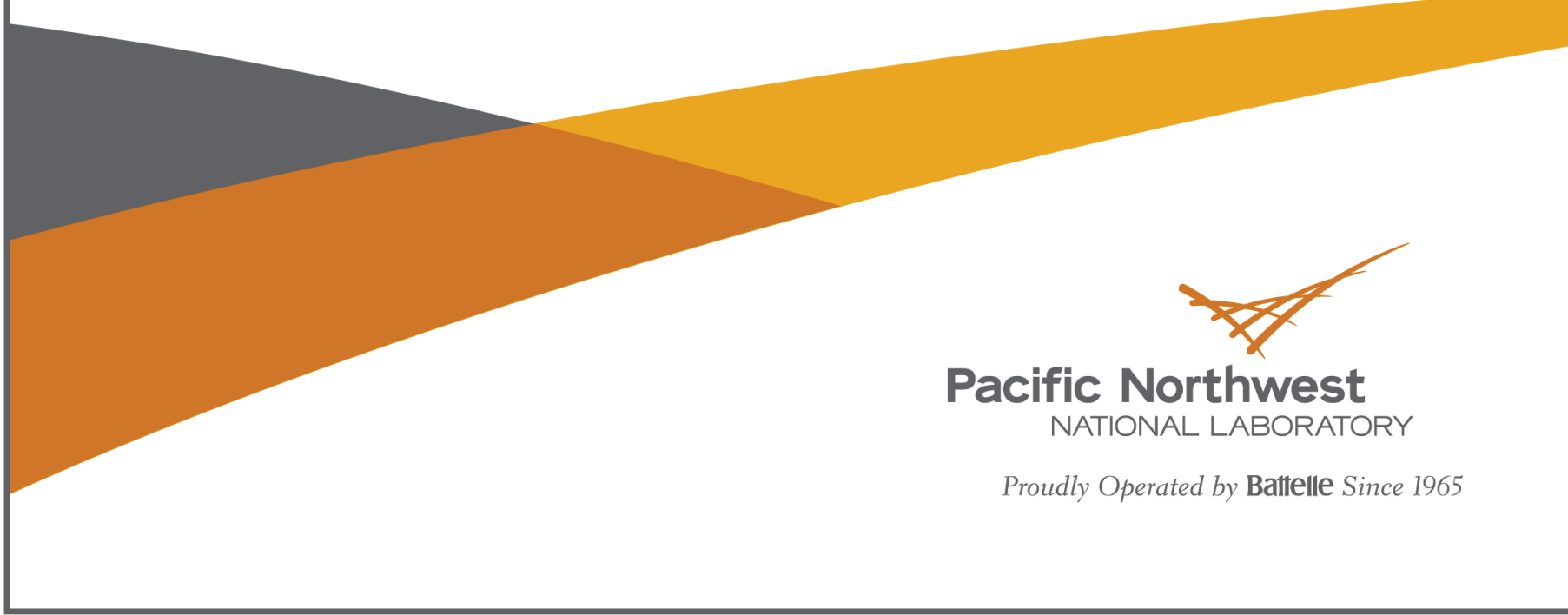




\title{
DISCLAIMER
}

This report was prepared as an account of work sponsored by an agency of the United States Government. Neither the United States Government nor any agency thereof, nor Battelle Memorial Institute, nor any of their employees, makes any warranty, express or implied, or assumes any legal liability or responsibility for the accuracy, completeness, or usefulness of any information, apparatus, product, or process disclosed, or represents that its use would not inf ringe privately owned rights. Reference herein to any specific commercial product, process, or service by trade name, trademark, manufacturer, or otherwise does not necessarily constitute or imply its endorsement, recommendation, or favoring by the United States Government or any agency thereof, or Battelle Memorial Institute. The views and opinions of authors expressed herein do not necessarily state or reflect those of the United States Government or any agency thereof.

\author{
PACIFIC NORTHWEST NATIONAL LABORATORY \\ operated by \\ BATTELLE \\ for the \\ UNITED STATES DEPARTMENT OF ENERGY \\ under Contract DE-AC05-76RL01830
}

Printed in the United States of America
Available to DOE and DOE contractors from the
Office of Scientific and Technical Information
P.O. Box 62, Oak Ridge, TN 37831-0062;
ph: (865) 576-8401
fax: $(865) 576-5728$
email: reports@adonis.osti.gov
Available to the public from the National Technical Information Service
5301 Shawnee Rd., Alexandria, VA 22312
ph: (800) 553-NTIS (6847)
email: orders@ntis.gov <http://www.ntis.gov/about/form.aspx>
Online ordering: http://www.ntis.gov

This document was printed on recycled paper. 


\title{
JSATS Decoder Software Manual
}

\section{FINAL REPORT}

\author{
AE Flory \\ BL LaMarche \\ MA Weiland
}

May 2013

Prepared for

U.S. Army Corps of Engineers, Portland District

under an Interagency Agreement with the U.S. Department of Energy

Contract DE-AC05-76RL01830

Pacific Northwest National Laboratory

Richland, Washington 99352 



\section{Preface}

The Juvenile Salmon Acoustic Telemetry System (JSATS) software user's manual presented here describes the installation and use of the JSATS decoder software. The software was developed for use with the JSATS cabled array system for decoding digitized acoustic signals. A complementary manual describes the use of the detector software used for acquiring the acoustic signals and recording them to file. This software was developed by the Pacific Northwest National Laboratory (PNNL) for the U.S. Army Corps of Engineers, Portland District to assist with estimating dam passage survival and other performance measures stipulated by the 2008 Federal Columbia River Power System Biological Opinion (BiOp) and the 2008 Columbia Basin Fish Accords.

Many others made significant contributions to development and testing of this software including Tom Carlson, Eric Choi, Daniel Deng, Jayson Martinez, Adam Flory, Tylor Abel, Yong Yuan, and Tom Seim. Thank you also to Joanne Duncan, Susan Ennor, George Batten, and Matt Hennen whose edits, comments, and improvements were much appreciated. 



\section{Acronyms and Abbreviations}

$\begin{array}{ll}\text { AMT } & \text { acoustic micro-transmitter } \\ b w m & \text { binary waveform format } \\ \text { CPU } & \text { central processing unit } \\ \text { CRC } & \text { cyclic redundancy check } \\ c s v & \text { comma separated variable } \\ \text { DSP } & \text { digital signal processing } \\ \text { FPGA } & \text { field programmable gate array } \\ \text { GPS } & \text { global positioning system } \\ \text { GUI } & \text { graphical user interface } \\ \text { I/O } & \text { input/output } \\ \text { JSATS } & \text { Juvenile Salmon Acoustic Telemetry System } \\ \text { ms } & \text { millisecond(s) } \\ \text { SNR } & \text { signal-to-noise ratio } \\ \text { TOA } & \text { time of arrival }\end{array}$





\section{Contents}

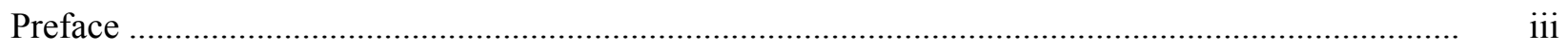

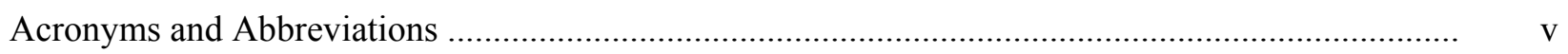

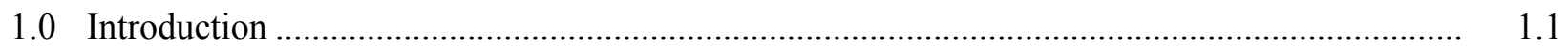

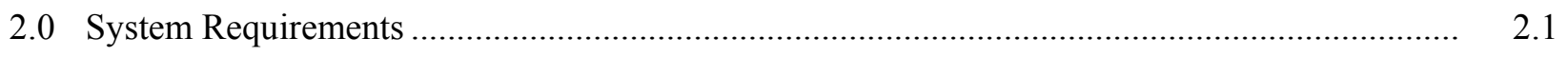

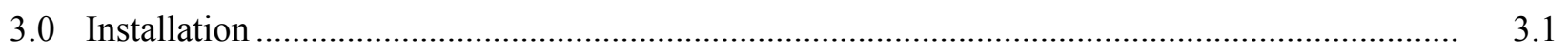

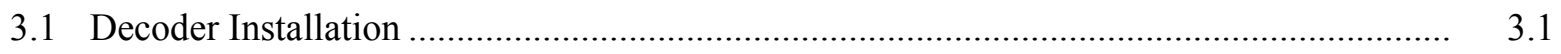

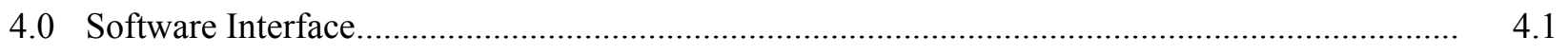

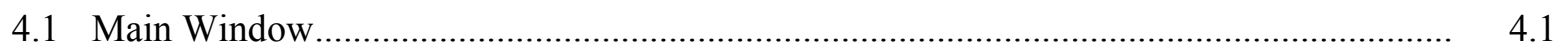

4.1.1 File Menu Bar............................................................................................ 4.1

4.1.2 Decoder Quick-Launch Bar.............................................................................. 4.3

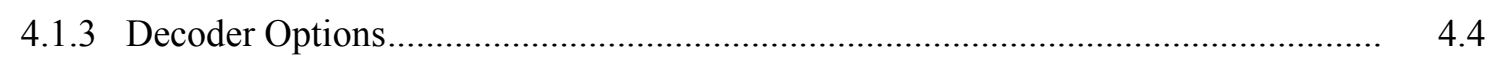

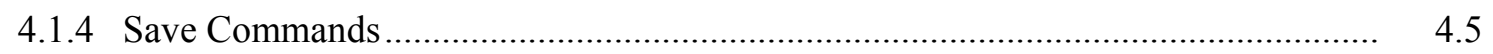

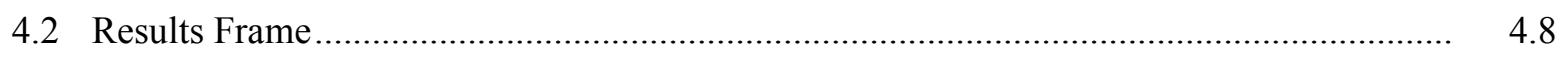

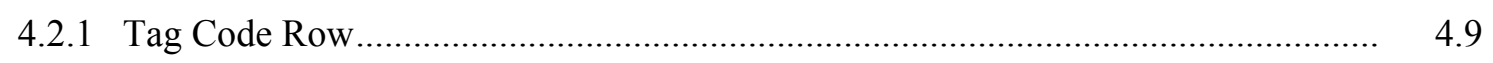

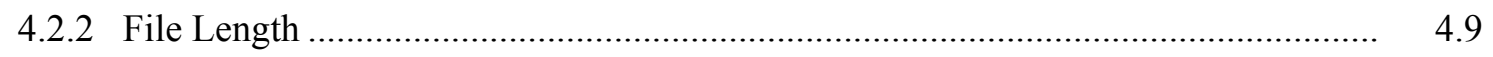

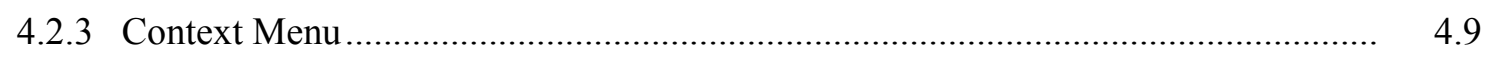

4.2.4 Viewing Tag Code Data Waveforms .............................................................. 4.10

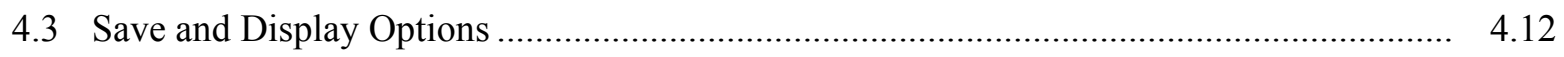

4.3.1 Save Only Valid Tagcodes Option ................................................................... 4.12

4.3.2 Show Only Valid Tagcodes Option................................................................... 4.12

4.3.3 Insert Results at Top of List Option .................................................................... 4.13

4.3.4 Display Tags Option..................................................................................... 4.13

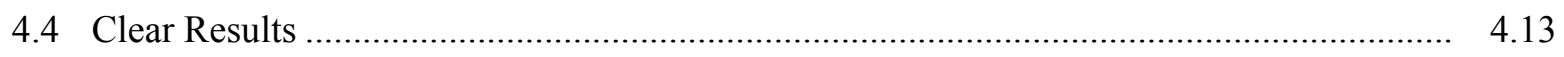

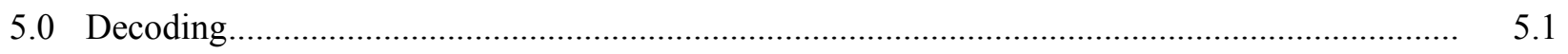

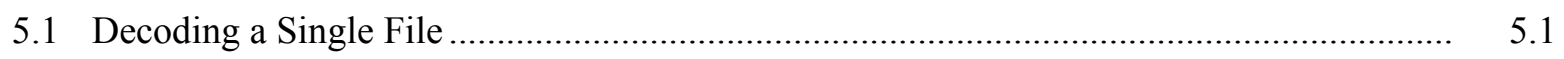

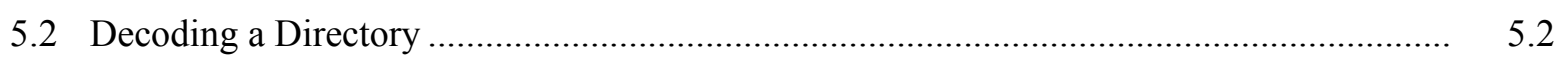

5.2.1 Selecting the Decoding Directory ................................................................. 5.3

5.2.2 Include Sub-Directories Option..................................................................... 5.4

5.2.3 Rename Extension After Decode Option ...................................................... 5.4

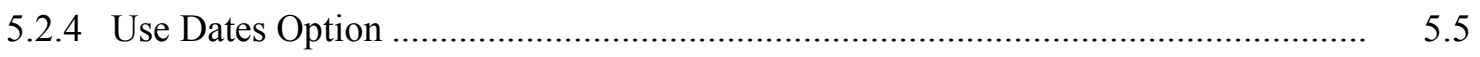

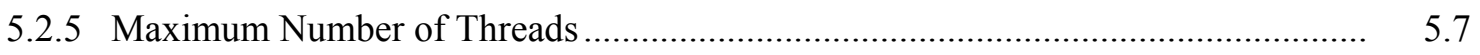

5.2.6 How Do I Know It Is Decoding?................................................................... 5.7

5.2.7 Stopping the Directory Decoding Process.......................................................... 5.8

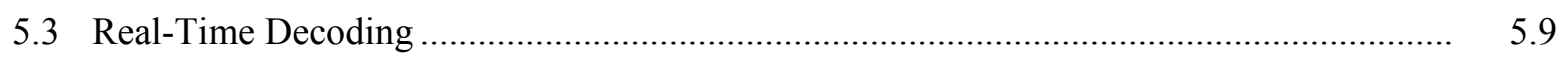

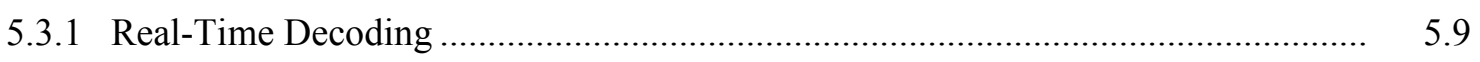

5.3.2 Include Sub-Directories........................................................................ 5.12 
5.3.3 Rename Extension After Decode ….................................................................. 5.12

5.3.4 Delete File After Decode............................................................................... 5.14

5.3.5 Milliseconds Before Decoding ...................................................................... 5.14

5.3.6 Maximum Number of Threads .................................................................... 5.14

5.3.7 Cache Expiration Time (mins) …................................................................. 5.15

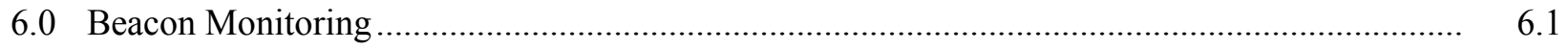

6.1 Statistics and Beacon Monitoring Window .................................................................. 6.1

6.2 Adding a Beacon to the Statistics and Beacon Monitoring List....................................... 6.2

6.3 Deleting a Single Beacon ......................................................................................... 6.3

6.4 Deleting All Beacons from the List........................................................................... 6.3

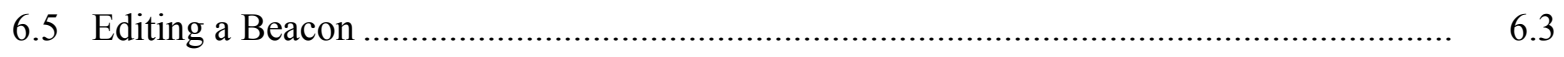

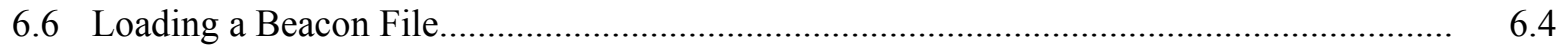

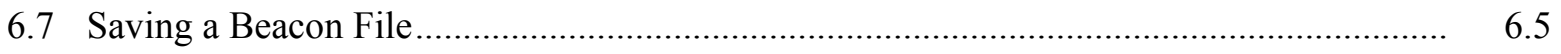

6.8 Setting Statistics Options and Saving Stats Options ..................................................... 6.6

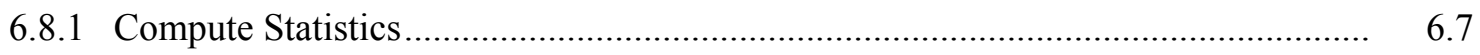

6.8.2 Time Period for Computing Statistics ............................................................ 6.7

6.8.3 Tag Codes Required to Compute Statistics ....................................................... 6.7

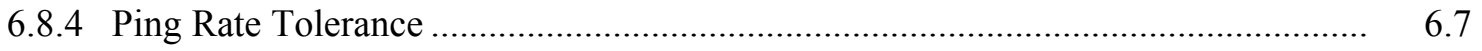

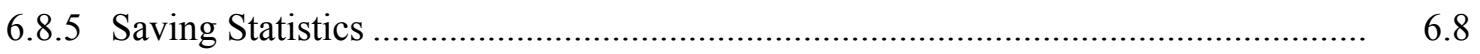

6.8.6 Saving Beacon Statistics ........................................................................ 6.8

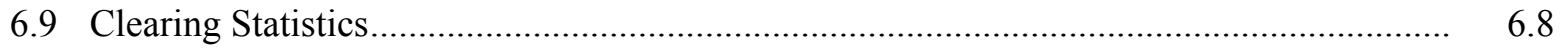

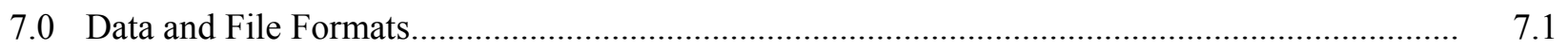

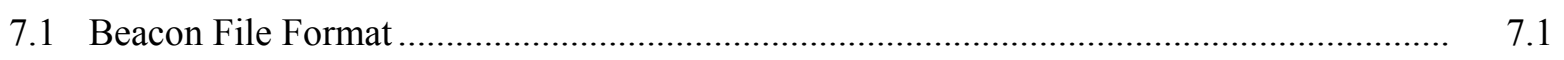

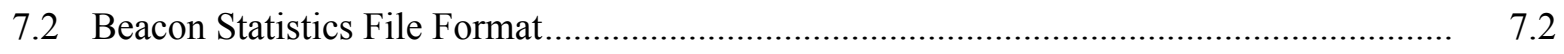

7.3 Decoder Results File Format ...................................................................................

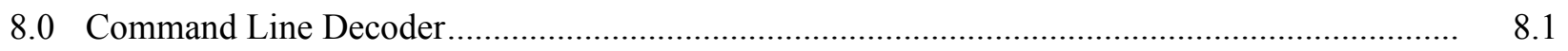

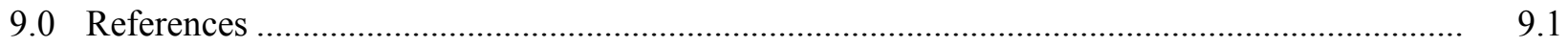




\section{Figures}

1.1 Flowchart Depicting the Flow of Data for the JSATS Cabled Receiver ................................. 1.1

1.2 Structure of the JSATS Cabled Array System and JSATS Software ...................................... 1.2

3.1 Windows Explorer Window Showing the JSATS Decoder Setup File .................................. 3.1

3.2 JSATS Decoder Setup Wizard Dialog Box .................................................................... 3.2

3.3 Enter or Browse to the Chosen Installation Folder Location within the Installation Folder

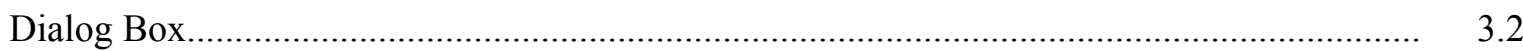

3.4 Select the Next Button in the Confirm Installation Dialog Box to Initiate the Install

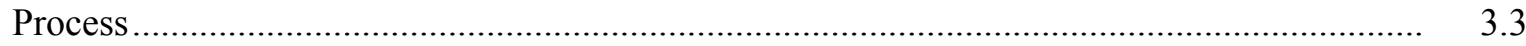

3.5 Installation Progress Will Be Shown in the Installing JSATS Decoder Dialog Box................ 3.3

3.6 The Successful Installation of the JSATS Decoder Will Be Shown in the Installation

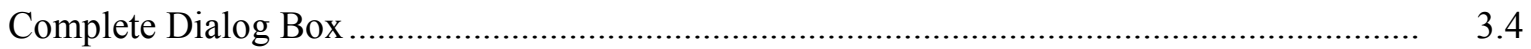

4.1 JSATS Decoder Main Application Window Showing the Menu Structure ............................

4.2 TDOA6a Operating Options Adjustment Panel .................................................................. 4.2

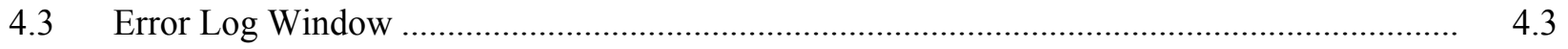

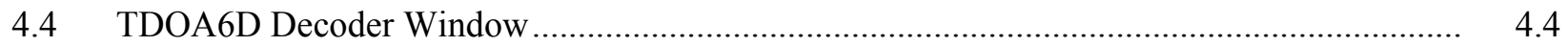

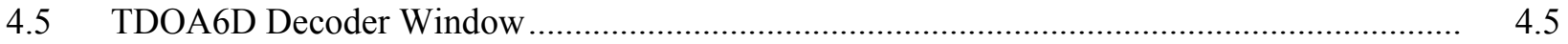

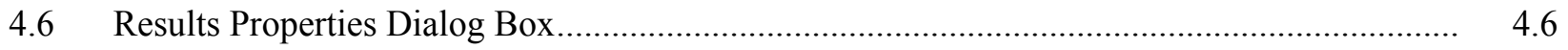

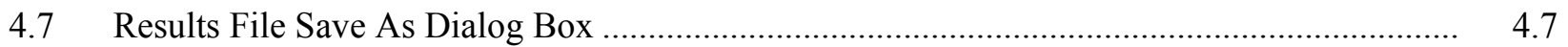

4.8 TDOA6D Results Are Shown in Spreadsheet Format in the Lower Frame of the Decoder

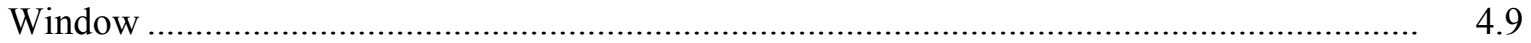

4.9 The Context Menu Is Revealed by Right-Clicking on the Results Spreadsheet...................... 4.10

4.10 Resize the Results Spreadsheet by Repositioning the Scroll Bar ............................................ 4.11

4.11 Resized Results Space after Repositioning the Scroll Bar ................................................... 4.11

4.12 Save and Display Options.................................................................................................. 4.12

4.13 Tag Code Values with a G Prefix Are Displayed when the Show Only Valid Tagcodes Option is Selected.

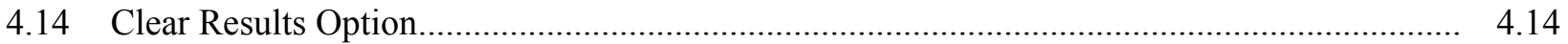

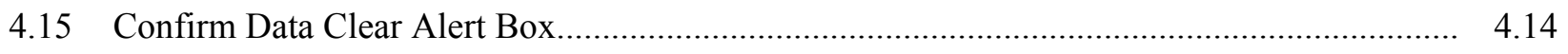

$5.1 \quad$ Decoder Command Bar Options................................................................................... 5.1

5.2 Decode File Open File Dialog Box .............................................................................. 5.2

$5.3 \quad$ Decoder Directory Options Dialog Box ......................................................................... 5.3

5.4 Decode Directory Browse for Folder Dialog Box .............................................................. 5.4

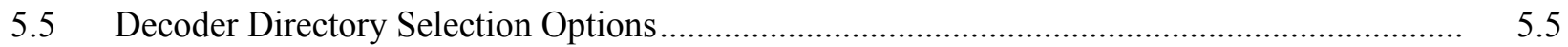

5.6 Selecting the Decode Start Date and Time from a . bwm File................................................ 5.6

5.7 Updating the Calendar Using Set From and Set To Times ................................................... 5.6

5.8 Select Ok to Start the Decoding Process ........................................................................... 5.7

5.9 Options at the Top of the Decoder Window Are Disabled during the Directory Decoding Process 
5.10 Select the Stop Decoding Button to Terminate a Directory Decode ................................... 5.9

5.11 Real-Time Decoding Option ............................................................................. 5.10

5.12 Real-Time Decoder Options Dialog Box ….................................................................. 5.10

5.13 Real-Time Decoder Directory Selection .................................................................... 5.11

5.14 Real-Time Decode Directory Browse for Folder Option Dialog Box ................................... 5.12

5.15 Real-Time Decoder Options Dialog Box ........................................................................ 5.13

5.16 The JSATS Decoder Configuration File Is Commonly Located in the Program Files

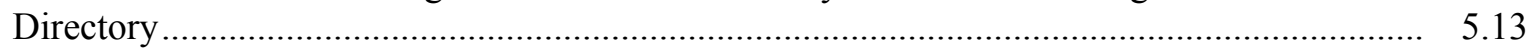

5.17 The File Extension for Renamed Files Can Be Changed by Modifying the Configuration

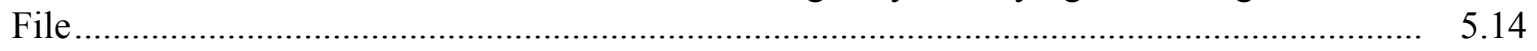

6.1 Statistics and Beacon Monitoring Options ….............................................................. 6.1

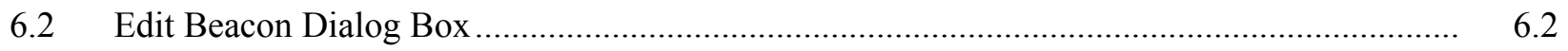

6.3 To Delete a Beacon, Highlight the Row and Select Delete .................................................. 6.3

6.4 The Confirmation Dialog Box to Delete All Beacons on the List........................................... 6.3

6.5 Beacon List File Example Using Notepad ..................................................................... 6.4

6.6 The Text File Containing Beacon Information Is Selected from the Open File Dialog Box .... 6.4

6.7 Beacon List Loaded from an ASCII Text File .................................................................. 6.5

6.8 When Saving a Beacon List the Save As Dialog Box Opens, Prompting Insertion of a File

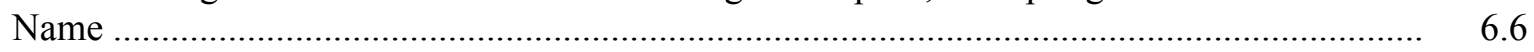

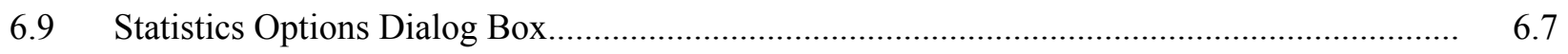

6.10 The Open Dialog Box where Beacon Statistics Will Be Saved ........................................... 6.8

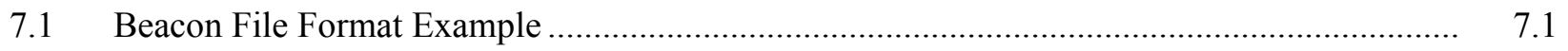

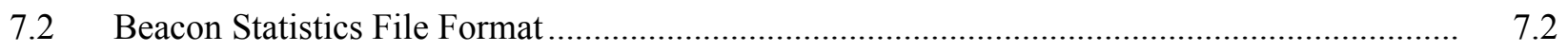

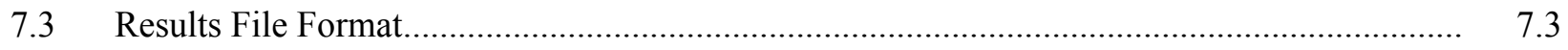




\subsection{Introduction}

The Juvenile Salmon Acoustic Telemetry (JSATS) system uses acoustic transmitters and receiving systems to track fish in one, two, or three dimensions (McMichael et al. 2010; Deng et al. 2011; Weiland et al. 2011). A modular JSATS array consists of software, a computer, multifunction electronic cards including a global positioning system (GPS) card, and digital signal processing (DSP) cards with field programmable gate array (FPGA) capability, a signal conditioning interface, four hydrophones, and four cables (Weiland et al. 2011). The conditioned signal enters the signal processing environment of the host computer where messages from JSATS transmitters are detected, decoded, and time stamped (Figure 1.1).

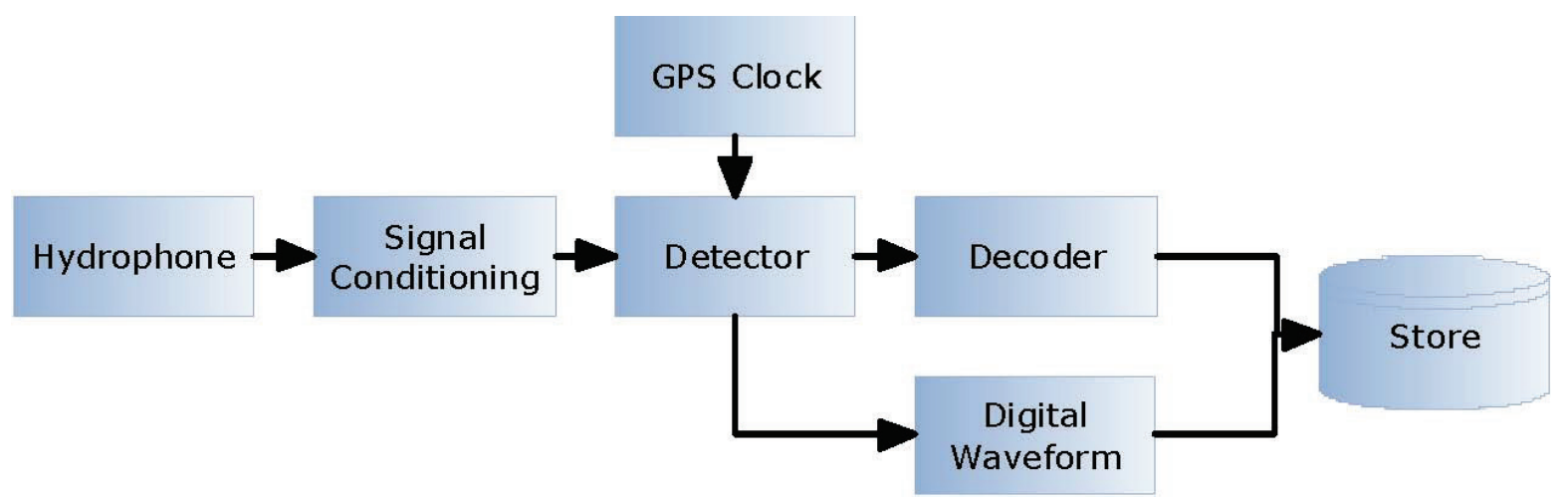

Figure 1.1. Flowchart Depicting the Flow of Data for the JSATS Cabled Receiver

The JSATS cabled array is an integrated system of off-the-shelf and custom components (Figure 1.2) designed for detection and tracking of fish tagged with acoustic micro-transmitters (AMTs) as they pass through the hydrophone array. This system was initially designed for use at hydroelectric facilities to track fish as they approach and pass the dam, but it can be adapted for many types of environments. The signal transmitted by the AMT implanted in a fish is detected by hydrophones deployed in known locations (surveyed positions). This signal is amplified by the hydrophone, or hydrophones, on which the signal is detected and passed through cables to a receiver where the signal is conditioned and further amplified. The signal is then passed to the DSP/FPGA card in the computer for signal verification and acceptance. The signal is paired with the time from the GPS card to provide microsecond accuracy and then the data are written to disk as a .bwm (binary waveform format) file. If the decoder is running in the real-time mode and is able to keep up with the signals coming in, the file is opened, decoded, and the tag code decoded from the acoustic waveform is written to a .csv (comma- separated variable) file for use in future analysis not described in this manual. If the real-time decoder is unable to keep up with the stream of incoming .bwm files or the data are not being decoded in real-time the .bwm files will be decoded later (post-processed) using the Decode Directory function described in Section 5.2.

The JSATS decoder, explained in detail in this manual, is a software application that converts a digitized acoustic signal (a waveform stored in .bwm files) into a list of potential JSATS AMT tag codes along with other descriptive data about the signal, including time of arrival (TOA) and signal-to-noise ratio (SNR). The software is capable of decoding single files, directories, and viewing raw acoustic waveforms. When coupled with the JSATS detector, the decoder is capable of decoding in real time and can also provide statistical information about acoustic beacons placed within the detection range of hydrophones within a JSATS array. This field manual details the features and functionality of the JSATS decoder software. 


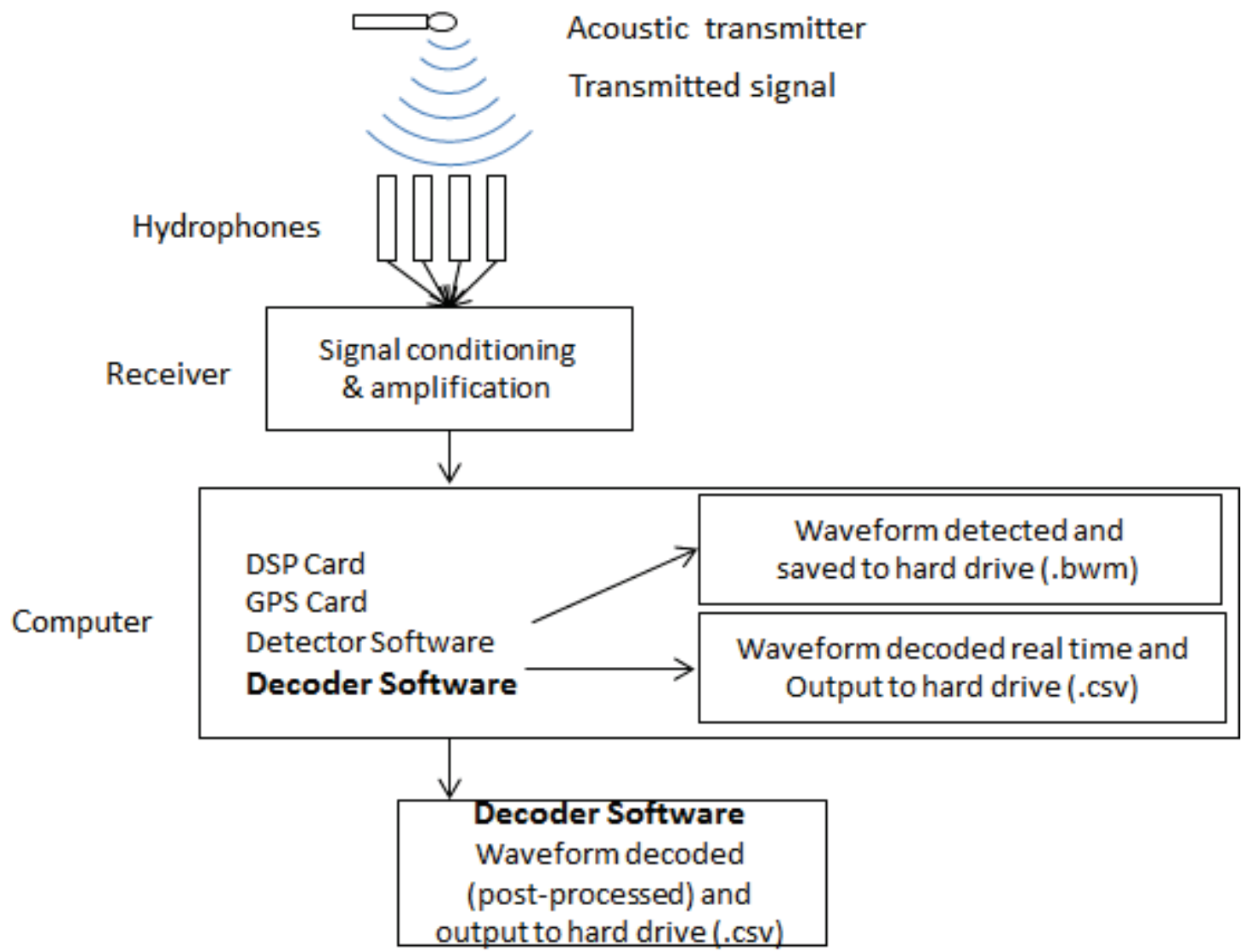

Figure 1.2. Structure of the JSATS Cabled Array System and JSATS Software

System requirements (Section 2.0) are presented first, followed by software installation instructions (Section 3.0), instructions for decoder setup (Section 4.0), decoding process initiation (Section 5.0), and monitoring of beacons using real-time decoding features (Section 6.0). Section 7.0 explains data and file format configurations, and Section 8.0 describes the Command Line decoder, a lightweight version of the JSATS decoder. 


\subsection{System Requirements}

This section provides information about the minimum system requirements for operation of the JSATS decoder.

\section{Hardware Requirements}

\section{Decoder only}

\begin{tabular}{lcc}
\hline & \multicolumn{2}{c}{ Requirement } \\
\cline { 2 - 3 } System Component & Minimum & Recommended \\
\hline Processor & $1 \mathrm{GHz}$ & $2 \mathrm{GHz}$ multicore or greater \\
RAM & $512 \mathrm{MB}$ & 4 GB or greater \\
Disk Space Installation & $2.5 \mathrm{~GB}$ & $2.5 \mathrm{~GB}$ \\
\hline
\end{tabular}

Additional disk space is required for storing decoded waveform data. The amount of hard drive space needed depends on the number of . $b w m$ files being decoded and the data stored in the individual.$b w m$ files.

\section{Decoder/Detector}

\begin{tabular}{lcc}
\hline & \multicolumn{2}{c}{ Requirement } \\
\cline { 2 - 3 } System Component & Minimum & Recommended \\
\hline Processor & Pentium IV & $2 \mathrm{GHz}$ multicore or greater \\
RAM & $1 \mathrm{~GB}$ & 4 GB or greater \\
Disk Space Installation & $2.5 \mathrm{~GB}$ & $2.5 \mathrm{~GB}$ \\
PCI-X slots & 3 & 3 \\
\hline
\end{tabular}

Additional disk space is required for acquisition of raw . $b w m$ files and storing decoded waveform data. The amount of hard drive space needed depends on the number of . bwm files being acquired and decoded and the data stored in the individual .bwm files.

\section{Operating System Requirements}

- Windows 7 (32- or 64-bit)

- Windows XP (Service Pack 2 or 3)

\section{Required Utility Packages}

- .net Framework 4.0 - (http://www.microsoft.com/download/en/details.aspx?id=17718) - link accessed on December 12, 2012.

- VC++ Redistributable 8.0 (the appropriate package for your computer's operating system, i.e., x86 for 32-bit operating systems and x64 for 64-bit systems). 
- 32-Bit - (http://www.microsoft.com/downloads/details.aspx?FamilyID=9b2da534-3e03-43918a4d-074b9f2bc1bf\&displaylang=en) - link accessed on December 12, 2012

- 64-Bit - (http://www.microsoft.com/downloads/details.aspx?familyid=90548130-4468-4BBC9673-D6ACABD5D13B\&displaylang=en) - link accessed on December 12, 2012 


\subsection{Installation}

This section provides instructions for installation of the JSATS decoder. The software packages listed in Section 2.0 must be installed prior to the installation of the JSATS decoder. Instructions for installation and use of the JSATS detector software are provided in a companion manual, the JSATS Detector Software Manual.

\subsection{Decoder Installation}

The JSATS decoder installation file "SetupJSATSDecoderOTR.msi" is available from http://jsats.pnnl.gov/Software/.

In Windows Explorer, start the installation by double-clicking the file "SetupJSATSDecoderOTR.msi" (Figure 3.1).

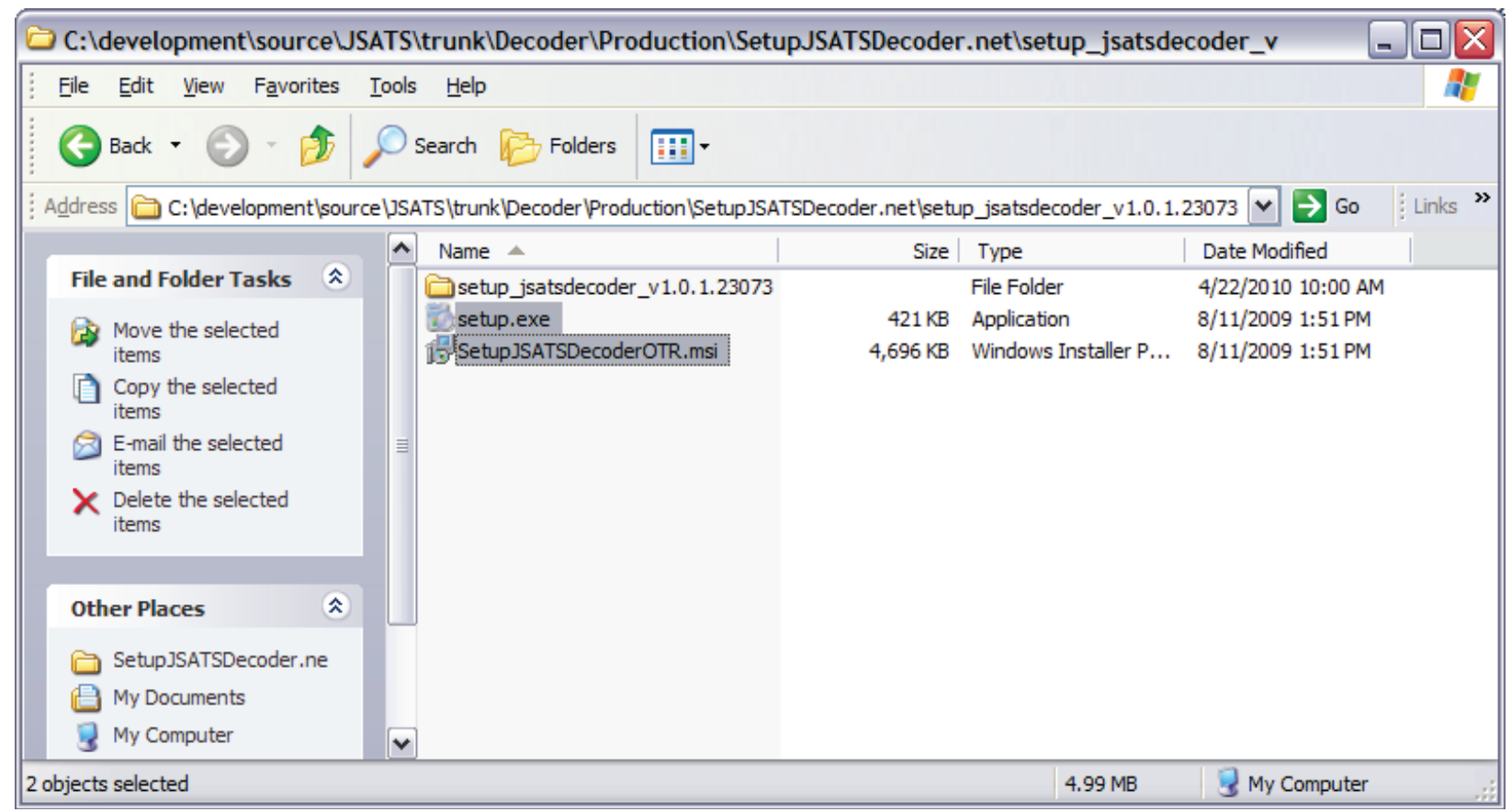

Figure 3.1. Windows Explorer Window Showing the JSATS Decoder Setup File

After the installer loads, you will be guided through a series of steps, beginning with the Setup Wizard dialog box (Figure 3.2). Select Next to continue installation. 


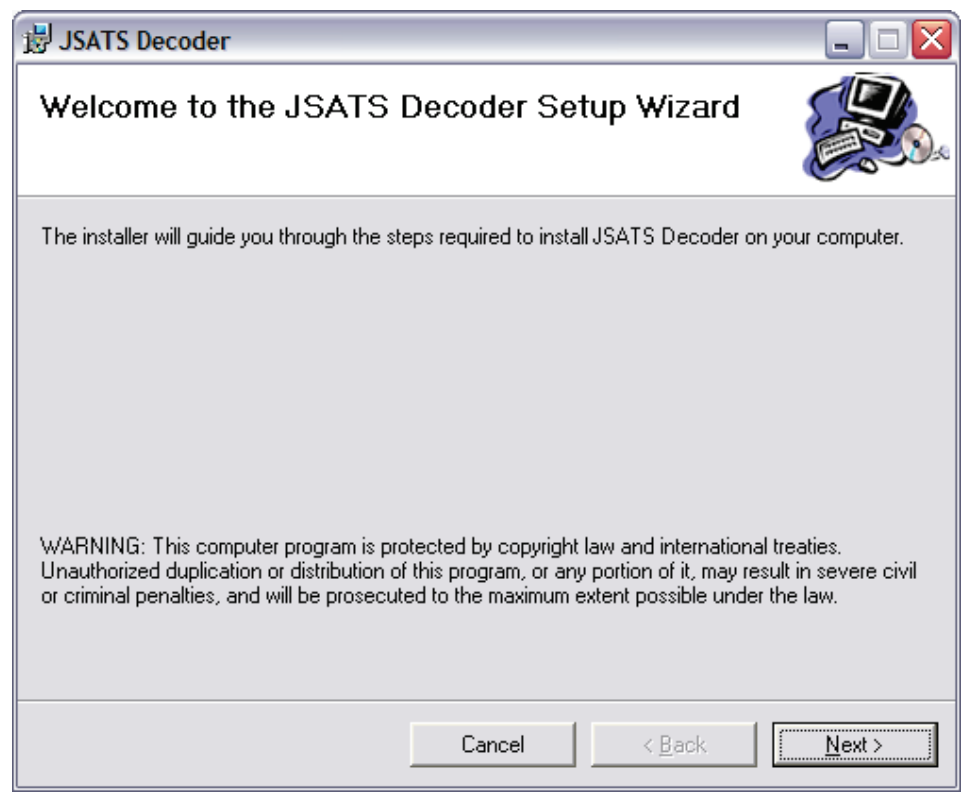

Figure 3.2. JSATS Decoder Setup Wizard Dialog Box

The first step of installation is to identify the folder in which you want to install the decoder. The default location for the installation folder is in the computer Program Files Directory, as shown in the Select Installation Folder dialog box in Figure 3.3. An alternative installation location may be entered in the Folder text box, or Browse can be selected to navigate to a designated folder location. Select the option button to choose whether or not the JSATS decoder will be available for others that may use the computer, and select Next.

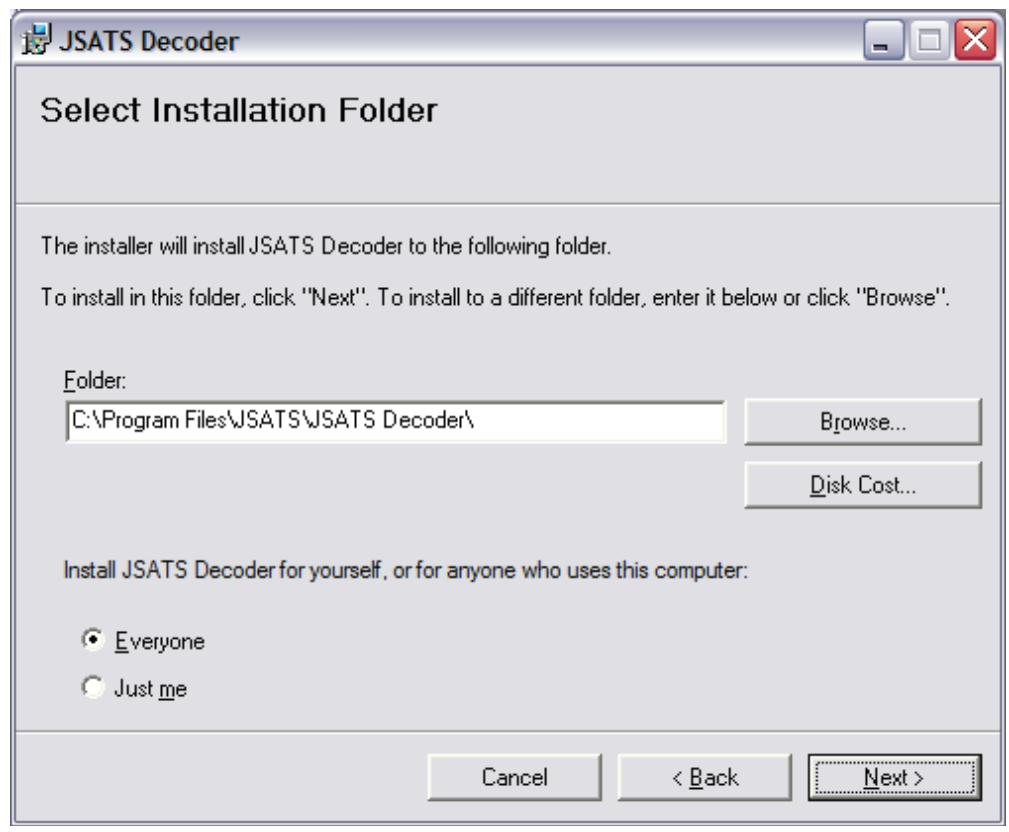

Figure 3.3. Enter or Browse to the Chosen Installation Folder Location within the Installation Folder Dialog Box (first install screen) 
The Select Installation Folder dialog box will close and the Confirm Installation dialog box (Figure 3.4) will open. Select Next to confirm that you want to install the decoder and to initiate the software installation.

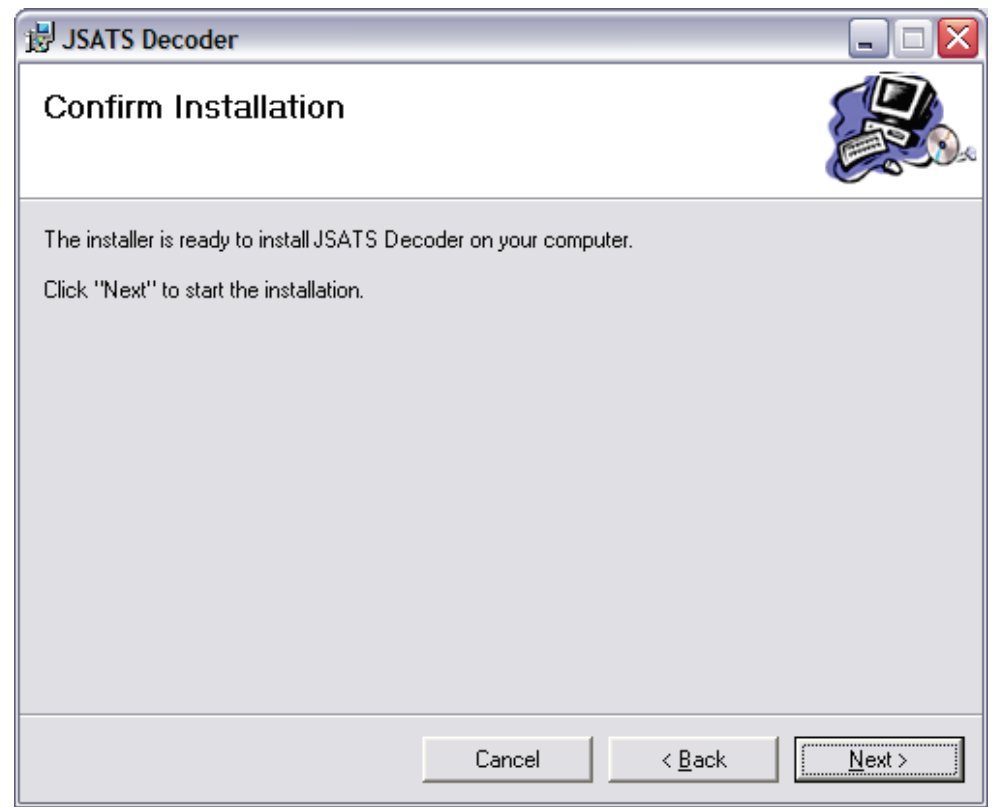

Figure 3.4. Select the Next Button in the Confirm Installation Dialog Box to Initiate the Install Process (second install screen)

The progression of the decoder installation will be indicated by the progress bar shown in the Installing JSATS Decoder dialog box (Figure 3.5). When completed, select Next.

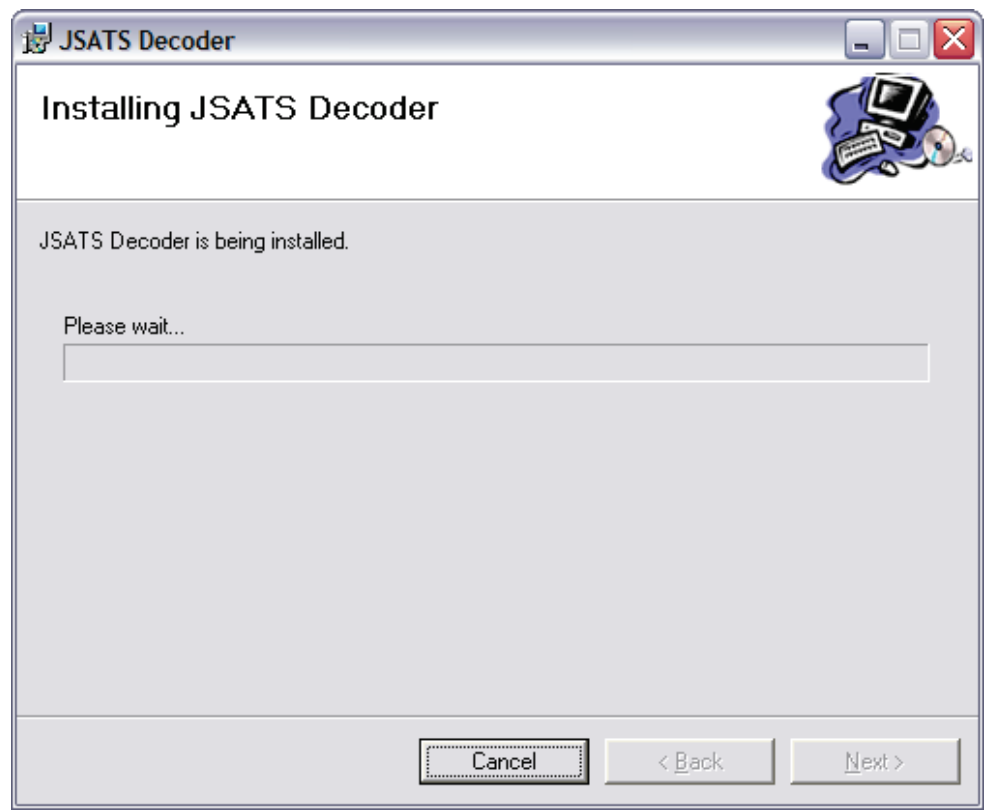

Figure 3.5. Installation Progress Will Be Shown in the Installing JSATS Decoder Dialog Box (third install screen) 
A new dialog box will open indicating the successful installation of the JSATS decoder (Figure 3.6); select the Close button to complete the installation process.

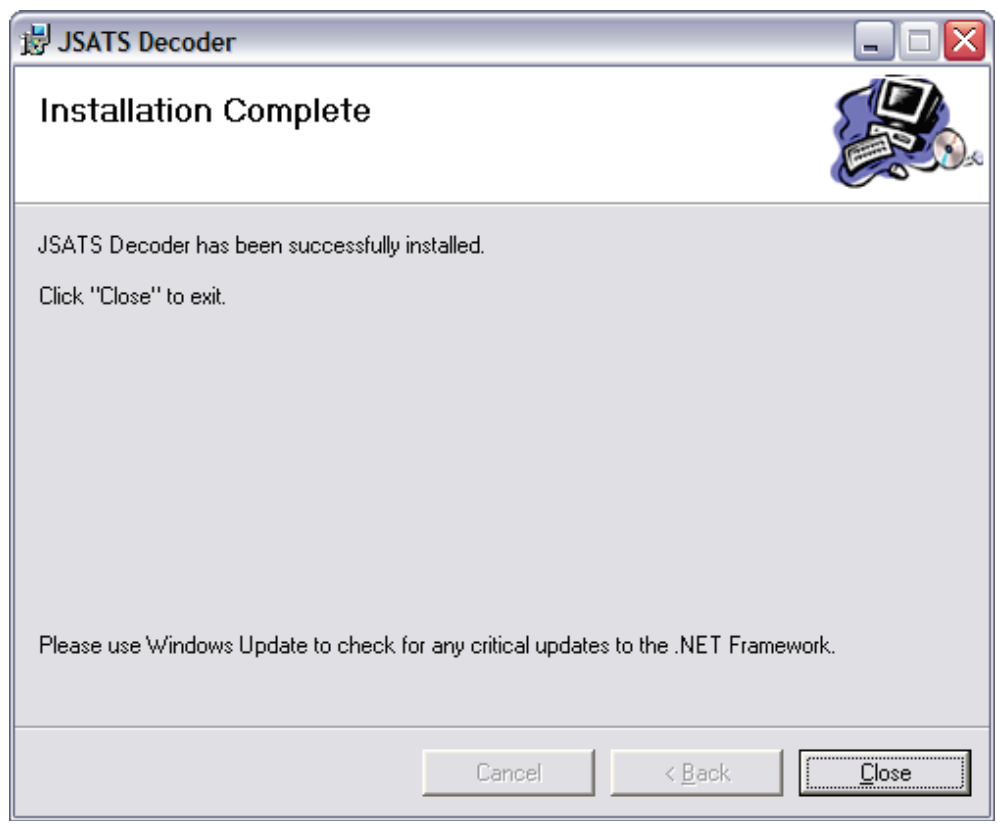

Figure 3.6. The Successful Installation of the JSATS Decoder Will Be Shown in the Installation Complete Dialog Box (final install screen). Select the Close button to complete the process. 


\subsection{Software Interface}

This section provides a descriptive overview of the layout and the function of the GUI (graphical user interface) and how to navigate the software to set preferences for viewing and decoding data.

\subsection{Main Window}

Figure 4.1 shows the layout of the main JSATS Decoder window. The menu structure is organized into five main categories (bordered in red): the File Menu Bar (Section 4.1.1), Decoder Quick Launch Bar (Section 4.1.2), Decoder Command Bar (Section 5.0), Decoder Options (Section 4.1.3), and Save Commands Bar (Section 4.1.4). Four of these elements are described in the following sections.

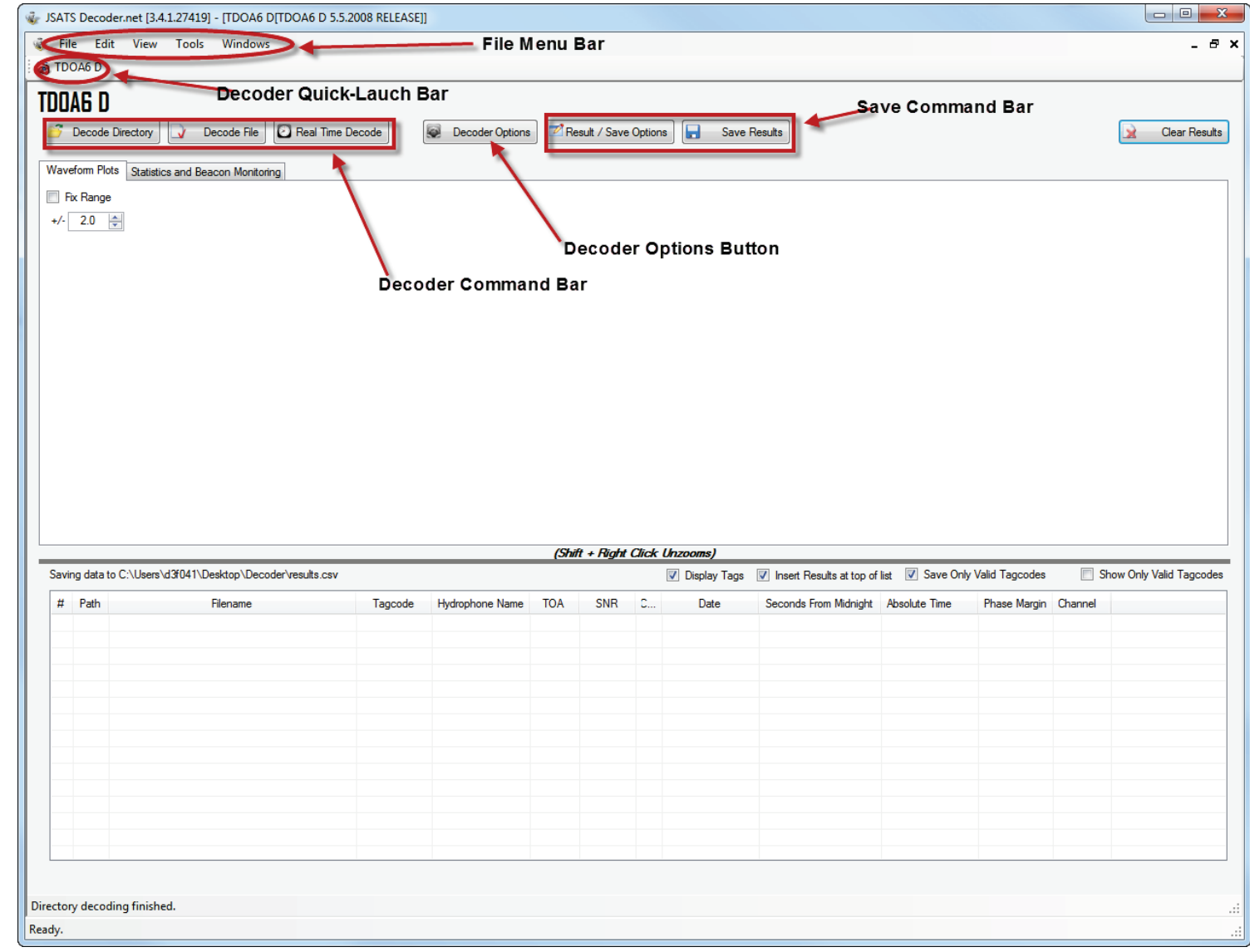

Figure 4.1. JSATS Decoder Main Application Window Showing the Menu Structure

\subsubsection{File Menu Bar}

The top level File Menu Bar contains several menus and sub-menus. 


\subsubsection{File Menu}

The File menu allows you to quit the program (Exit), save results (Save, Save As), and decode . $b w m$ files (Decode File, Decode Directory). The Decode File and Decode Directory options are described in Section 5.0.

\subsubsection{Edit Menu}

The Edit menu allows you to change decoder options. The decoder available with this software package, TDOA6D, has options that can be modified to optimize the processing of saved tag waveforms or detector output. The default options described have been tested and optimized for detector algorithm performance. To adjust decoder parameters, select the specific decoder (i.e., TDOA6D) options in the dropdown Edit menu, which will open the decoder operation adjustment panel (Figure 4.2). The options and preset parameters, shown as TDOA6a Options in Figure 4.2, are identical to TDOA6D options. It is recommended that these options not be adjusted without thoroughly testing the changes. The variables in the Options dropdown menu are not discussed in this manual.

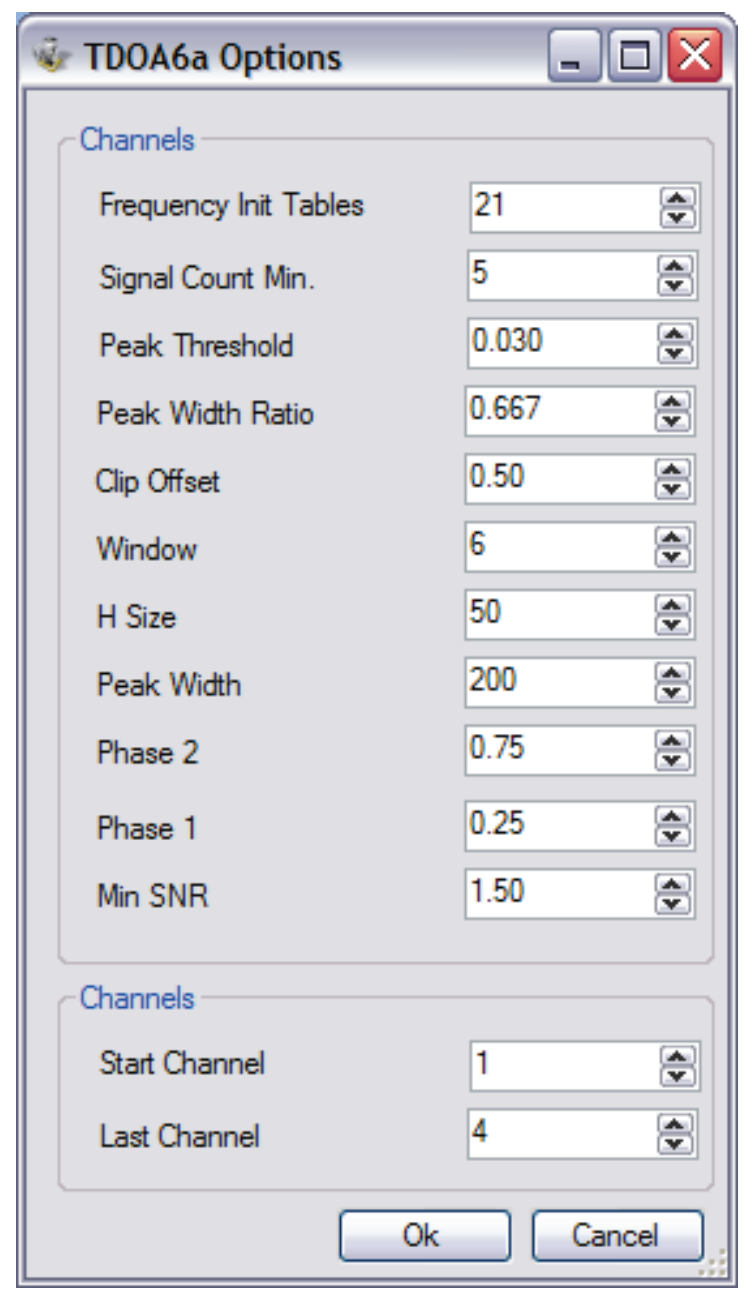

Figure 4.2. TDOA6a Operating Options Adjustment Panel 


\subsubsection{View Menu}

The View menu allows you to hide the status bar or to display application log or error messages. When application errors occur, they will be displayed in the Error Log window (Figure 4.3). Click on the Clear button in the lower right corner of the screen to clear the log file or click on $\mathbf{O K}$ to return to the main application screen.

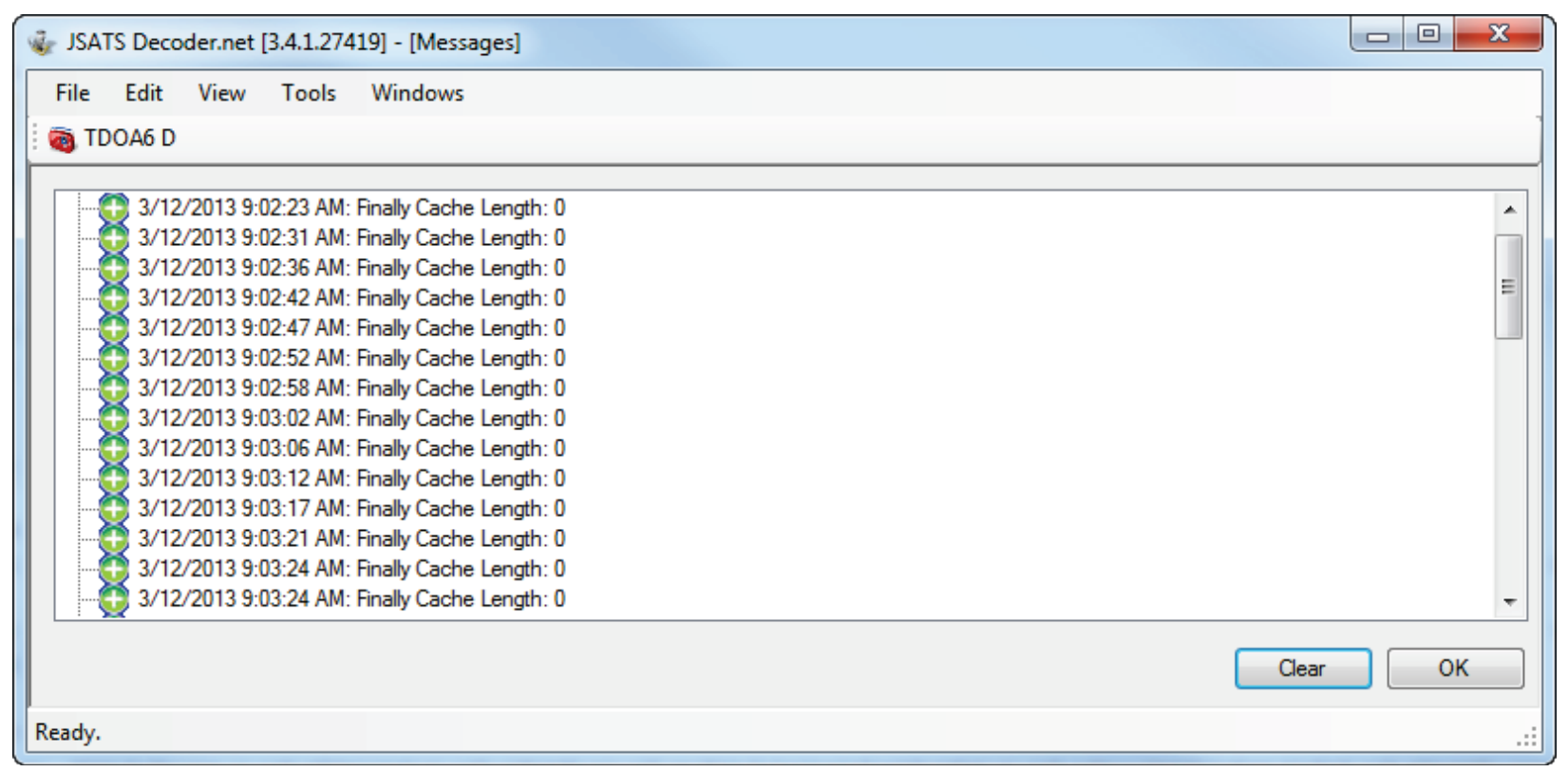

Figure 4.3. Error Log Window

\subsubsection{Tools Menu}

The Tools menu allows you to load other decoders not packaged with this software. The JSATS decoder system is plug-in based to permit developers to install and test new decoders without having to construct a user interface or other features. The extension of this decoder through plug-ins is not discussed in this user manual.

\subsubsection{Windows Menu}

The Windows menu displays a list of the decoder and log windows currently loaded, allowing the user to toggle through the available views.

\subsubsection{Decoder Quick-Launch Bar}

The JSATS decoder should automatically load one of the decoder windows upon startup; currently TDOA6D is the only decoder version loaded when the software is installed. If a decoder window is not loaded and opened, you can select the decoder from the decoder quick-launch bar by clicking on the decoder name (Figure 4.1).

When you select the TDOA6D decoder, the window shown in Figure 4.4 will open. 


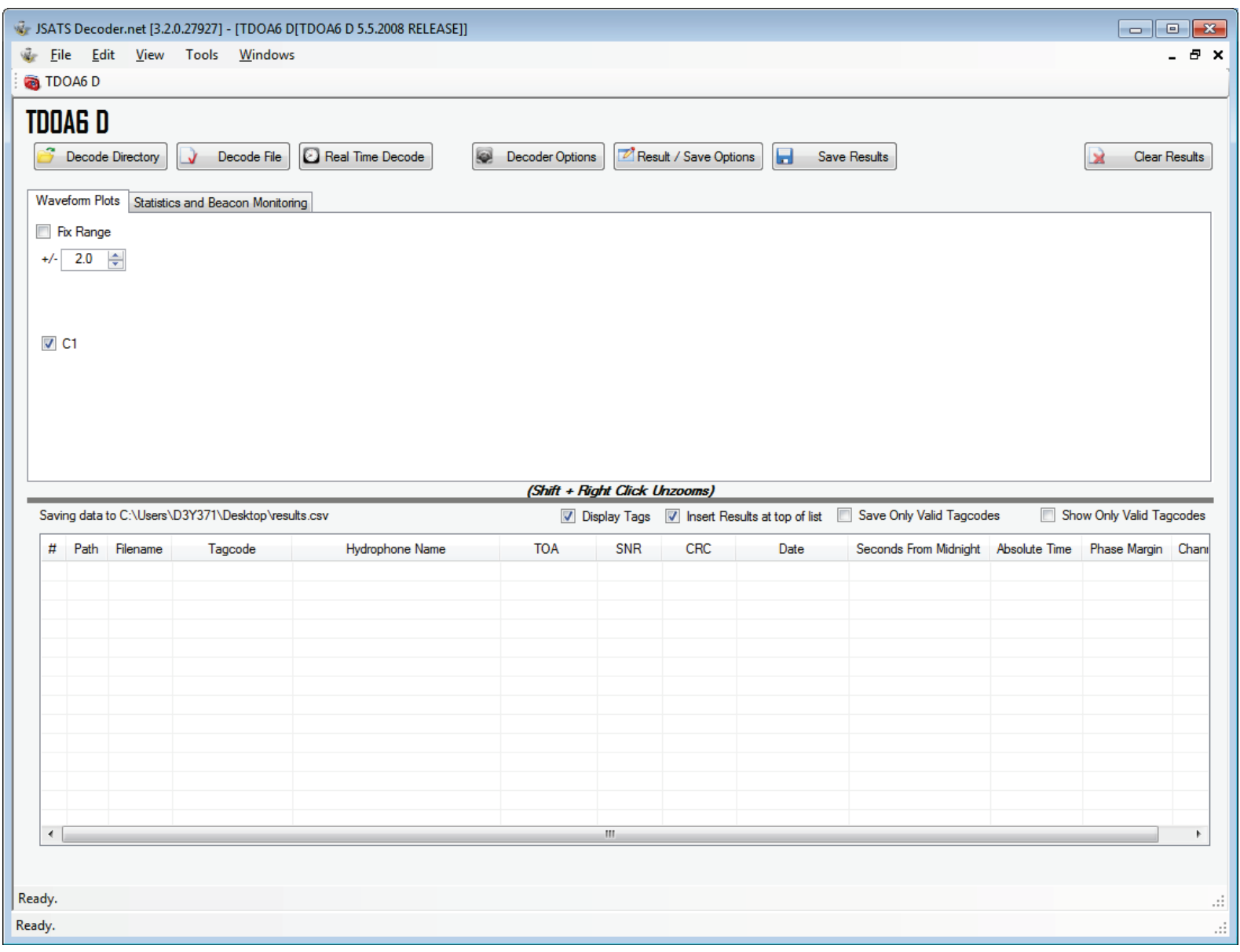

Figure 4.4. TDOA6D Decoder Window

\subsubsection{Decoder Options}

Each decoder has options that can be adjusted to control its performance. To adjust decoder parameters click the Decoder Options button in the main Decoder window (Figure 4.1).

Decoder operation options can also be changed by selecting the Edit menu, and then selecting the Decoder Options item as described in Section 4.1.2.

After changing the decoder operation parameters, select $\mathbf{O K}$ to accept the changes, or Cancel to reject any modifications. The default options have been thoroughly tested and verified in the lab and field. Any changes made to settings should be tested before they are implemented. Improper setting adjustment may result in reduction in decoding efficiency and other undesirable effects on decoder performance. 


\subsubsection{Save Commands}

\subsubsection{Result/Save Options}

The Result/Save Options menu allows you to specify where result files will be saved. When the decoder decodes a directory (in real time or during post-processing), the results are saved to a file in a .csv format. In the Result/Save Options menu you can adjust save parameters and display properties. To set these program parameters, click on the Result/Save Options button (Figure 4.5).

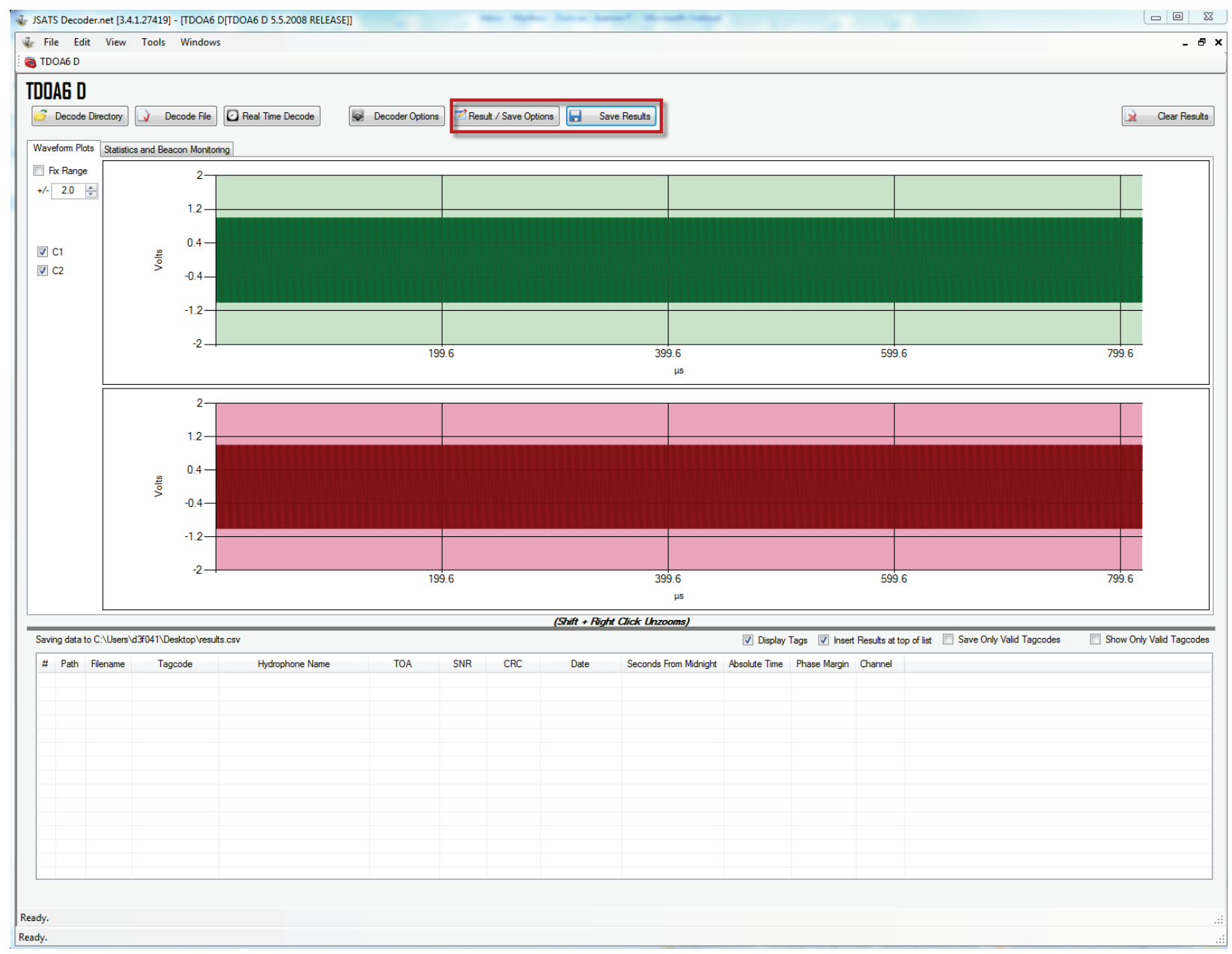

Figure 4.5. TDOA6D Decoder Window (with the Results/Save Options and Save Results buttons outlined in red)

After selecting the Result/Save Options menu item, the Results Properties dialog box shown in Figure 4.6 will appear. 


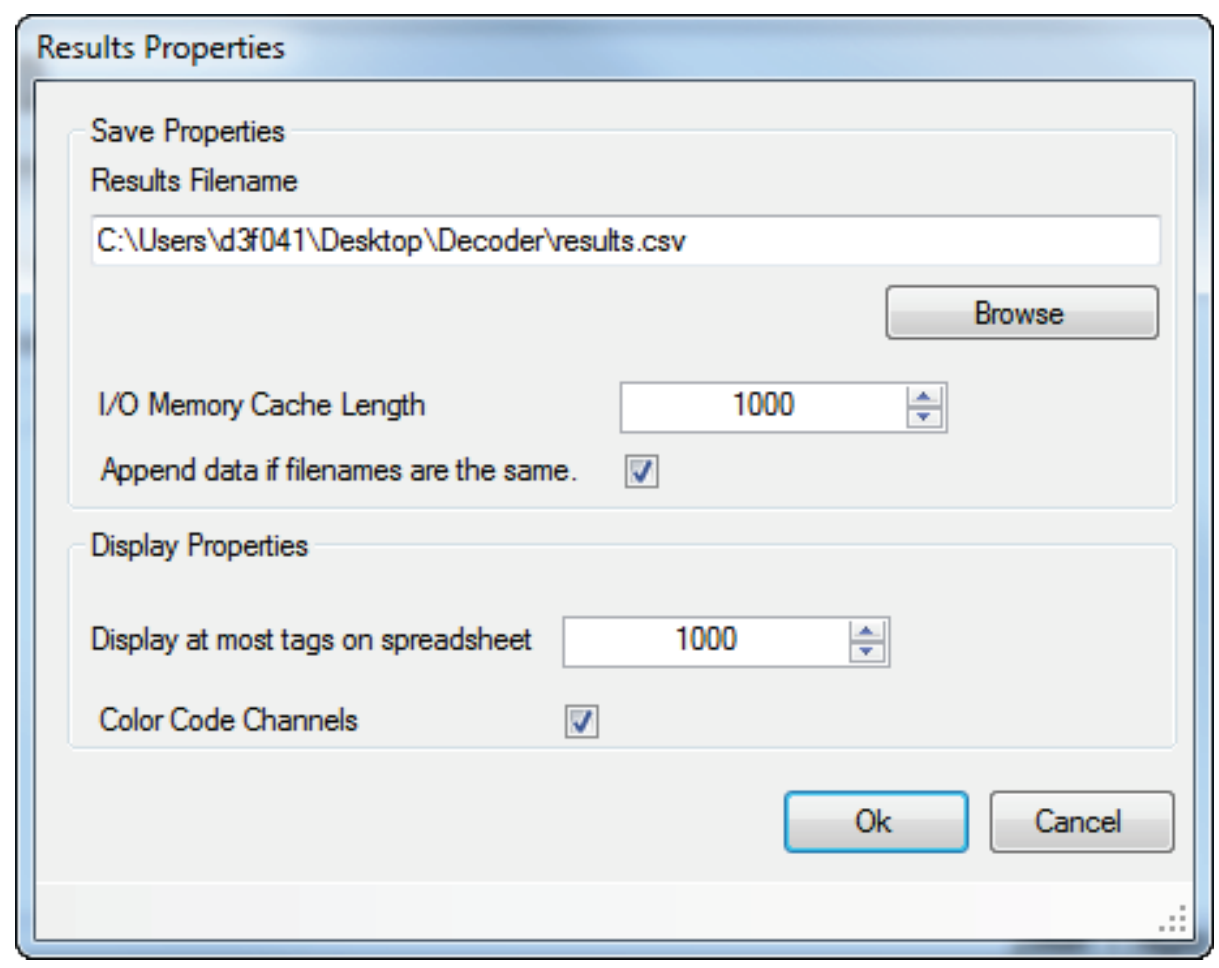

Figure 4.6. Results Properties Dialog Box

Enter preferred values in the Results Properties dialog box, which are described in the next subsections, and select $\mathbf{O K}$ to accept or Cancel to reject changes.

\section{Results Filename}

The Results Filename text box in the Results Properties dialog box specifies two things: the directory where the file will be saved and the base file name. For example, Figure 4.6 shows that the file results.csv will be saved to the $C: \mid$ Users $|d 3 f 041|$ Desktop $\mid$ Decoder directory.

The name of each results file created by the decoder program is unique in the sense that all data will not be saved into a single file with the name "results.csv"; instead, the decoder will create a unique file name by appending the date the data were processed and the directory where the decoded file was located to the output file name. This feature assists with data management. For example, if files located in directory "c:|templjsatsStudy" were decoded on April 22, 2010, the output file name assigned by the decoder would be

$$
\text { Results_20100422_1_jsatsStudy.csv. }
$$

To have the decoder append a number to the Results Filename when data are reanalyzed, check the Append data if file names are the same box (Figure 4.6). The decoder will append the new data to those already in the file instead of creating a new file for the reanalysis, keeping the file name the same as the original, as shown below:

Results_20100422_1_jsatsStudy.csv. 
The directory location can be edited by entering a new directory path and file name into the Filename text box or by clicking the Browse button, which will bring up the file Save As dialog box (Figure 4.7).

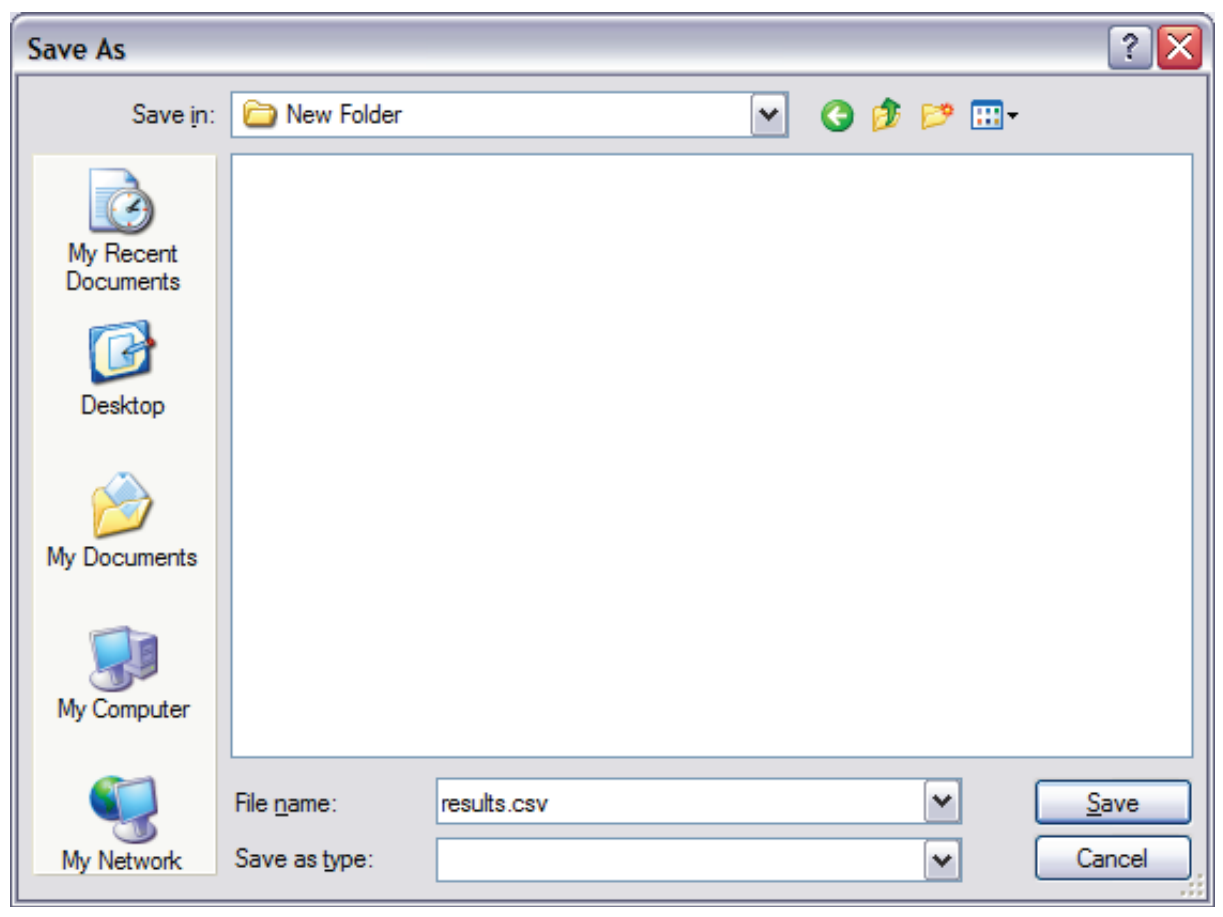

Figure 4.7. Results File Save As Dialog Box

Navigate to the directory to which you wish to save your results files, then enter the file name to be saved as described above and click Save.

\section{Input/Output Memory Cache Length}

As the decoder runs it handles several files (post-processing, real-time) and performs many file saving and file management operations. These input/output (I/O) operations can significantly slow processing because of computer disk performance inefficiency during access. The I/O Memory Cache Length parameter (Figure 4.6) allows the decoder to retain tag code results in a memory buffer until the buffer is filled to its specified capacity. Then the program performs a single block operation to write results to the disk. This reduces the number of disk access operations during processing and improves performance. By default, the decoder buffer is set to hold 1000 tag codes before writing the data to file.

\section{Display at Most Tags on Spreadsheet}

The Display at Most Tags on Spreadsheet feature, which is accessible through the Results Properties dialog box (Figure 4.6), permits the modification of the maximum number of tag codes displayed in the results spreadsheet list. By default, this number is 1000 . The tag codes displayed will be those most recently obtained by the program. Increasing this number will greatly reduce decoder performance by consuming computer resources. 


\section{Color Code Channels}

The Color Code Channels checkbox option allows data channels to be displayed in color (green, red, yellow, blue) or grayscale format. This feature confirms that acoustic data are being received on individual hydrophones during program setup (Figure 4.6).

\subsubsection{Save Results}

The decoder automatically saves the results as it processes acoustic signals.

\subsection{Results Frame}

The Results frame, shown in the lower half of the JSATS Decoder window, displays a spreadsheet listing tag codes and other relative data, obtained when . $b w m$ files are decoded (Figure 4.8). Information displayed in this spreadsheet includes the following:

- \# (a decode index value)

- Path (the directory for the processed file)

- Filename (name of the.$b w m$ file)

- Tagcode (prefixed with G for good, B for bad based on checksum values)

- Hydrophone Name (the name for the hydrophone set in the detector software and contained in the processed file)

- TOA (time of arrival)

- SNR (signal-to-noise ratio)

- CRC (cyclic redundancy check). Checksum computed over the 16-bit binary representation of the tag code

- Date (date acquired)

- Seconds from Midnight (when the file was created)

- Absolute Time (seconds from midnight plus the TOA)

- Phase Margin (a confidence metric for binary phase-shift keying phase transitions)

- Channel. 


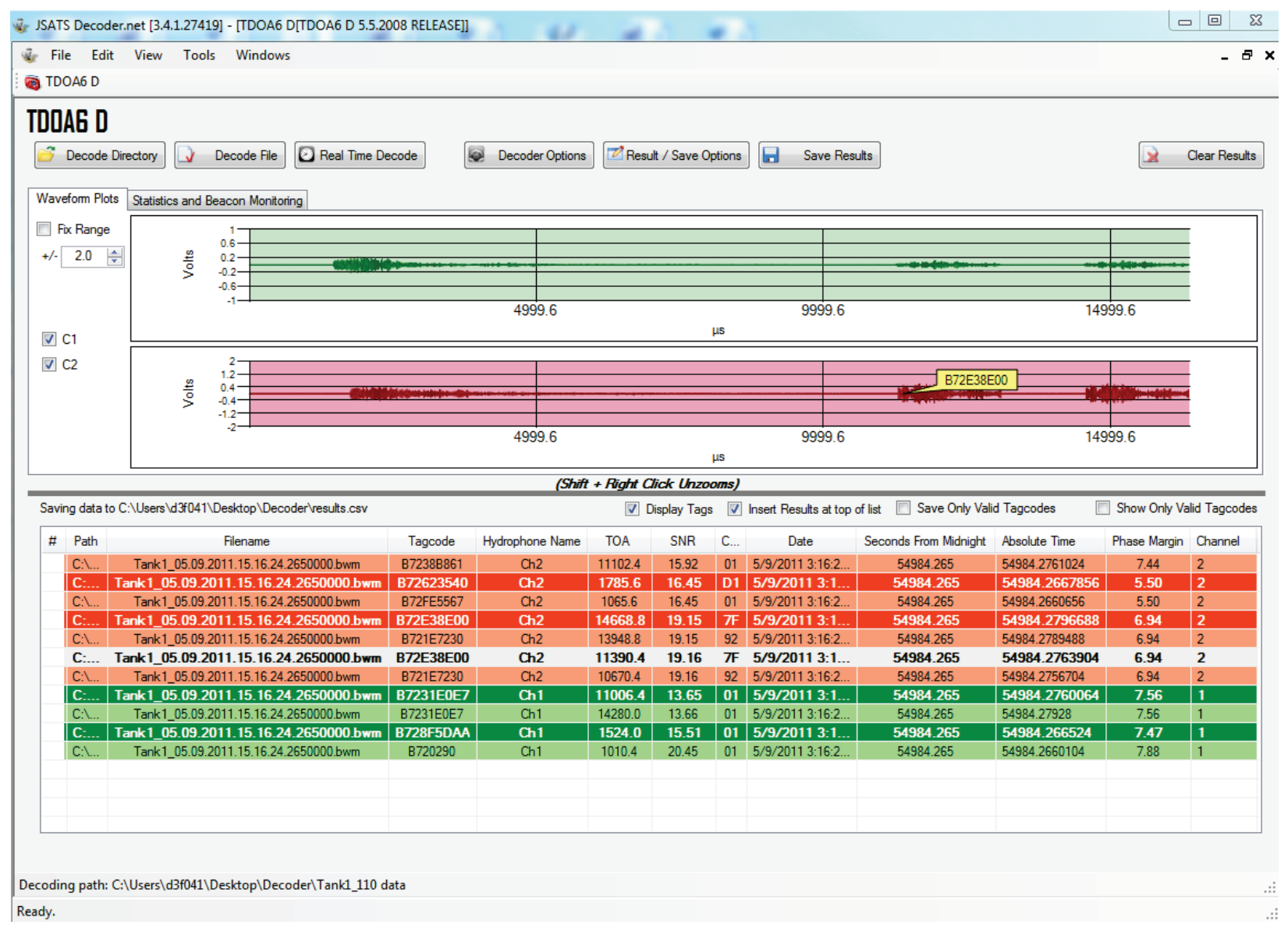

Figure 4.8. TDOA6D Results Are Shown in Spreadsheet Format in the Lower Frame of the Decoder Window

\subsubsection{Tag Code Row}

Selecting one of the rows in the Results spreadsheet will open the acoustic waveform plot in the visualization window above the spreadsheet (Figure 4.8). The decoded tag's relative position in the waveform plot and decoded code will be indicated.

\subsubsection{File Length}

The decoder will calculate several potential tag codes per file. The results derived from the decoding are best estimates of the potential tag codes present in the file waveform. As a result, there may be multiple tag code lines in the output spreadsheet with the same file name and possibly the same tag code, distinguished from one another by different TOA values. Multiple tag code lines will increase the .csv file length.

\subsubsection{Context Menu}

Right-clicking within the Results spreadsheet opens a Context menu (Figure 4.9). From the Context menu one or more tag codes can be selected and added to the beacon monitoring list by selecting the 
Monitor Tagcode option (explained further in Section 6.0); the Results Properties can be edited (Section 4.1.4.1); or the waveform plot for a specific tag code can be displayed.

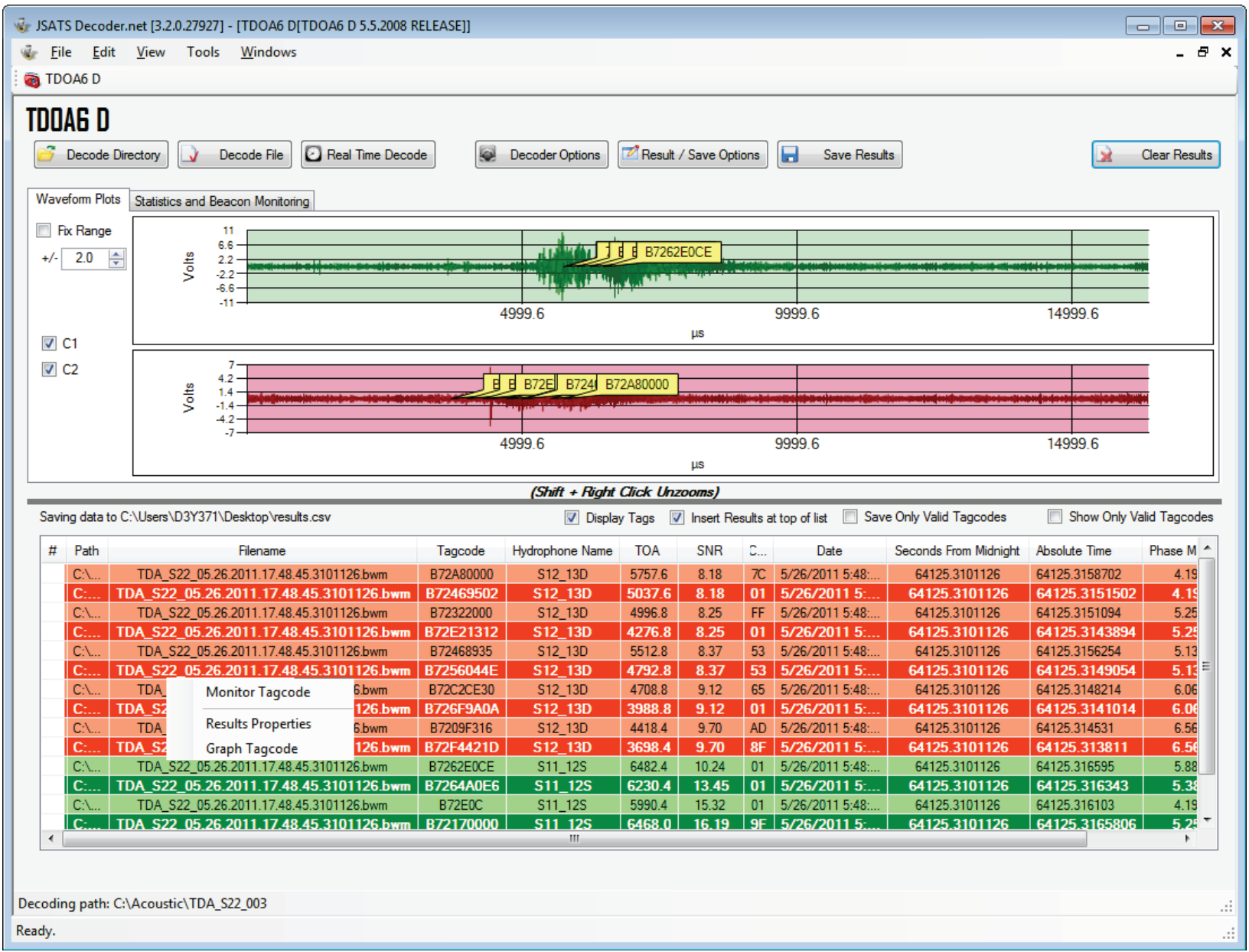

Figure 4.9. The Context Menu Is Revealed by Right-Clicking on the Results Spreadsheet

\subsubsection{Viewing Tag Code Data Waveforms}

If waveforms are not visible within the Results frame, the window can be expanded or reduced by clicking and dragging the thick gray scroll bar (highlighted in red in Figure 4.10 and Figure 4.11) to provide more or less space to display tag code data. 


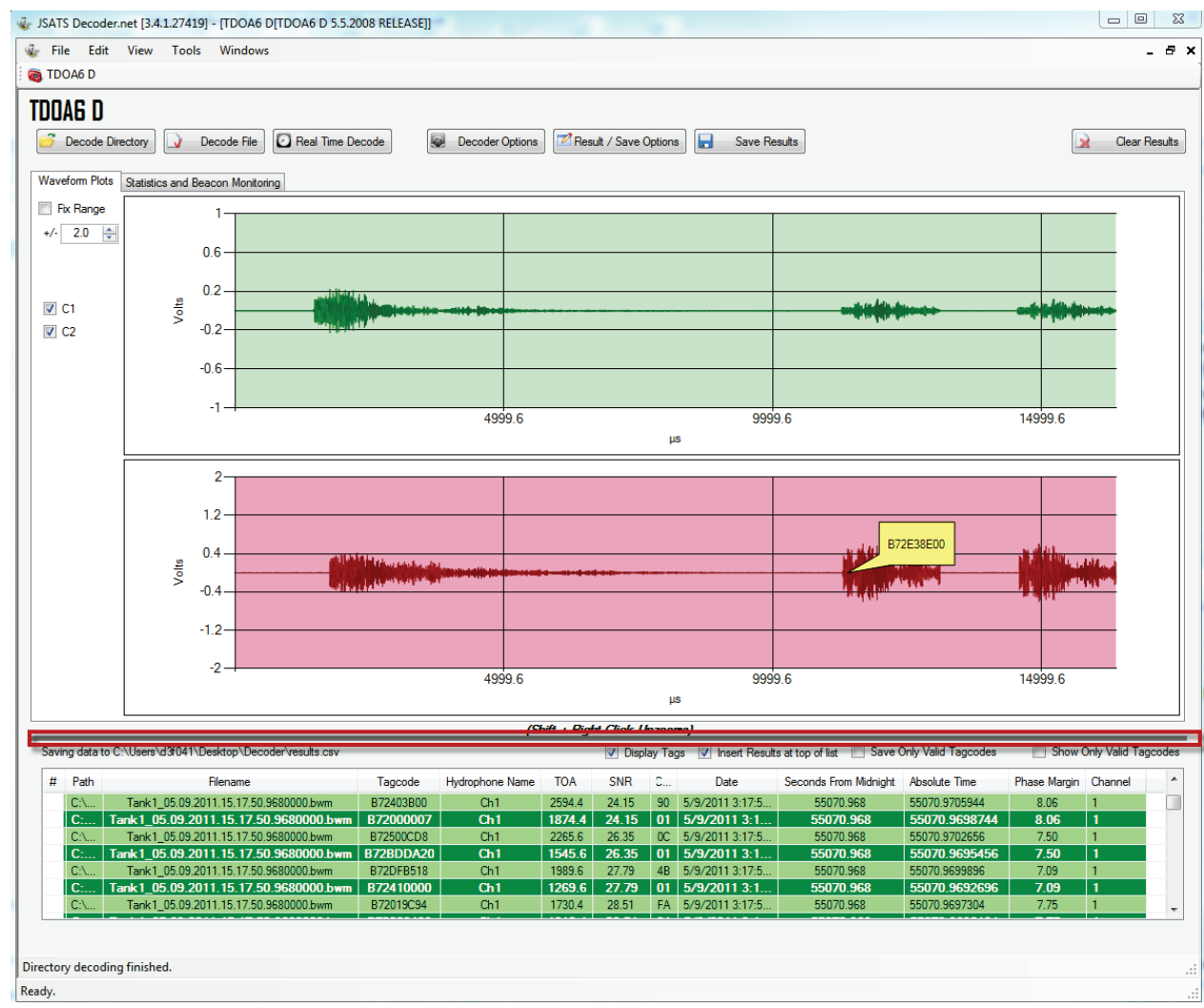

Figure 4.10. Resize the Results Spreadsheet by Repositioning the Scroll Bar (highlighted in red)

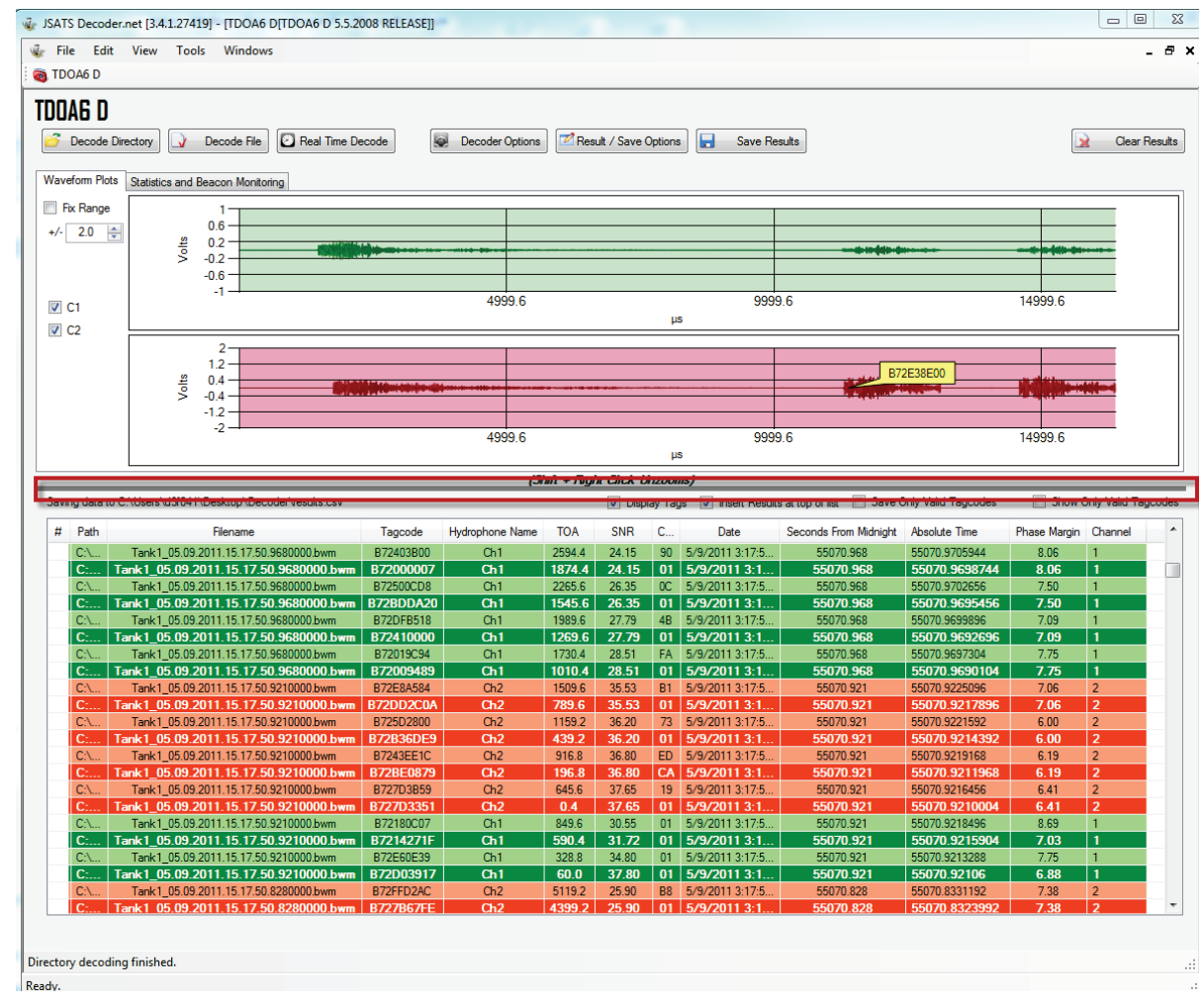

Figure 4.11. Resized Results Space after Repositioning the Scroll Bar 


\subsection{Save and Display Options}

Several options available within the decoder program for saving and visualizing data (Figure 4.12) are discussed in the following sections.

\subsubsection{Save Only Valid Tagcodes Option}

If the Save Only Valid Tagcodes box is checked (Figure 4.12), the decoder will only save tag codes that have a $\mathrm{G}$ prefix. The $\mathrm{G}$ indicates that a tag code has passed the checksum test.

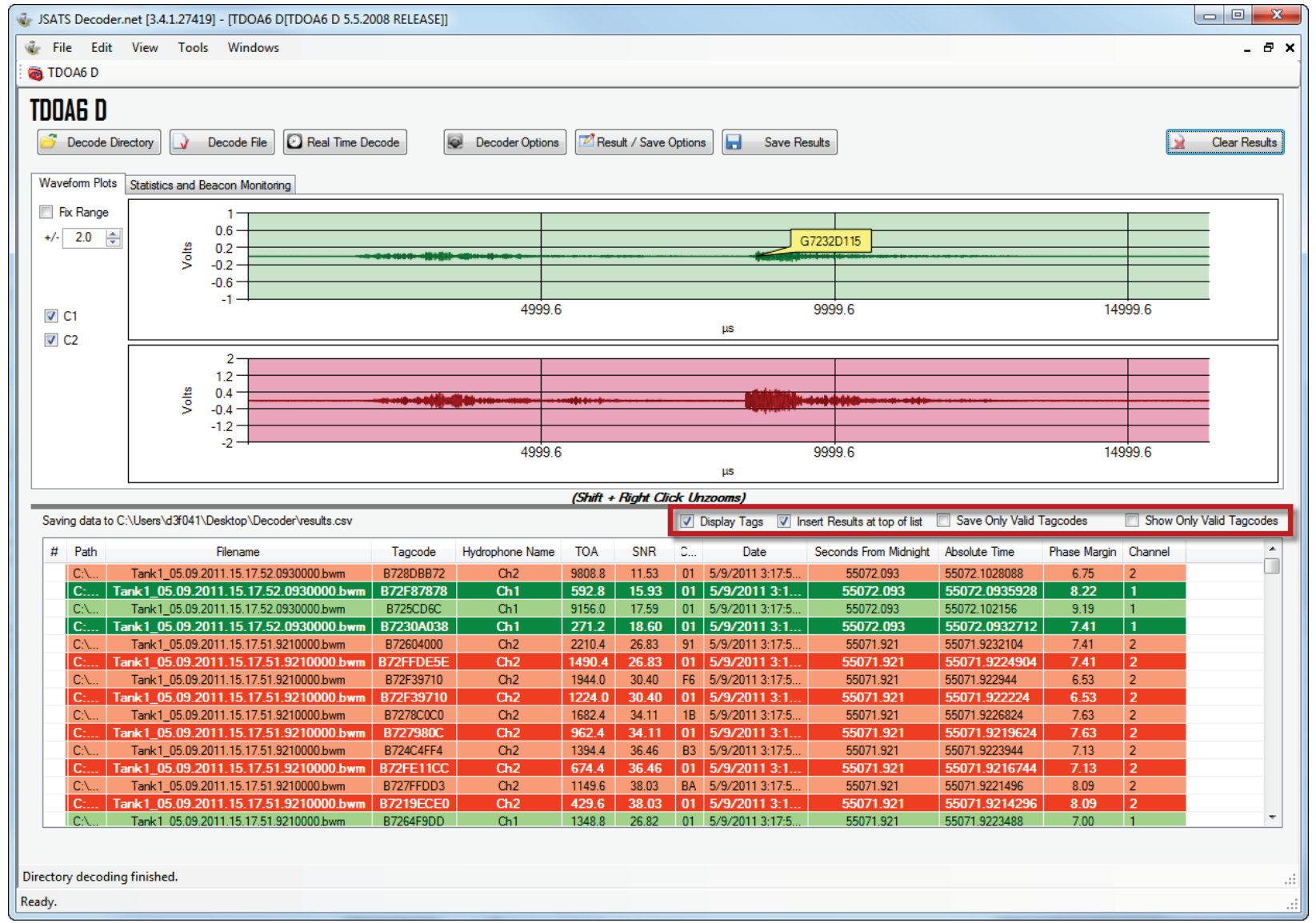

Figure 4.12. Save and Display Options (highlighted in red)

\subsubsection{Show Only Valid Tagcodes Option}

Checking the Show Only Valid Tagcodes box (highlighted in Figure 4.13) instructs the decoder to only display tag codes in the result window spreadsheet that have a $G$ prefix. The $G$ indicates the code passed the checksum test. If this box is checked, and the Save Only Valid Tagcodes box is not checked (Section 3.7.1), all tag codes will be saved even though only valid tag codes are displayed. This program feature reduces the number of potentially invalid codes that are displayed and is very useful for monitoring the system in the field. 


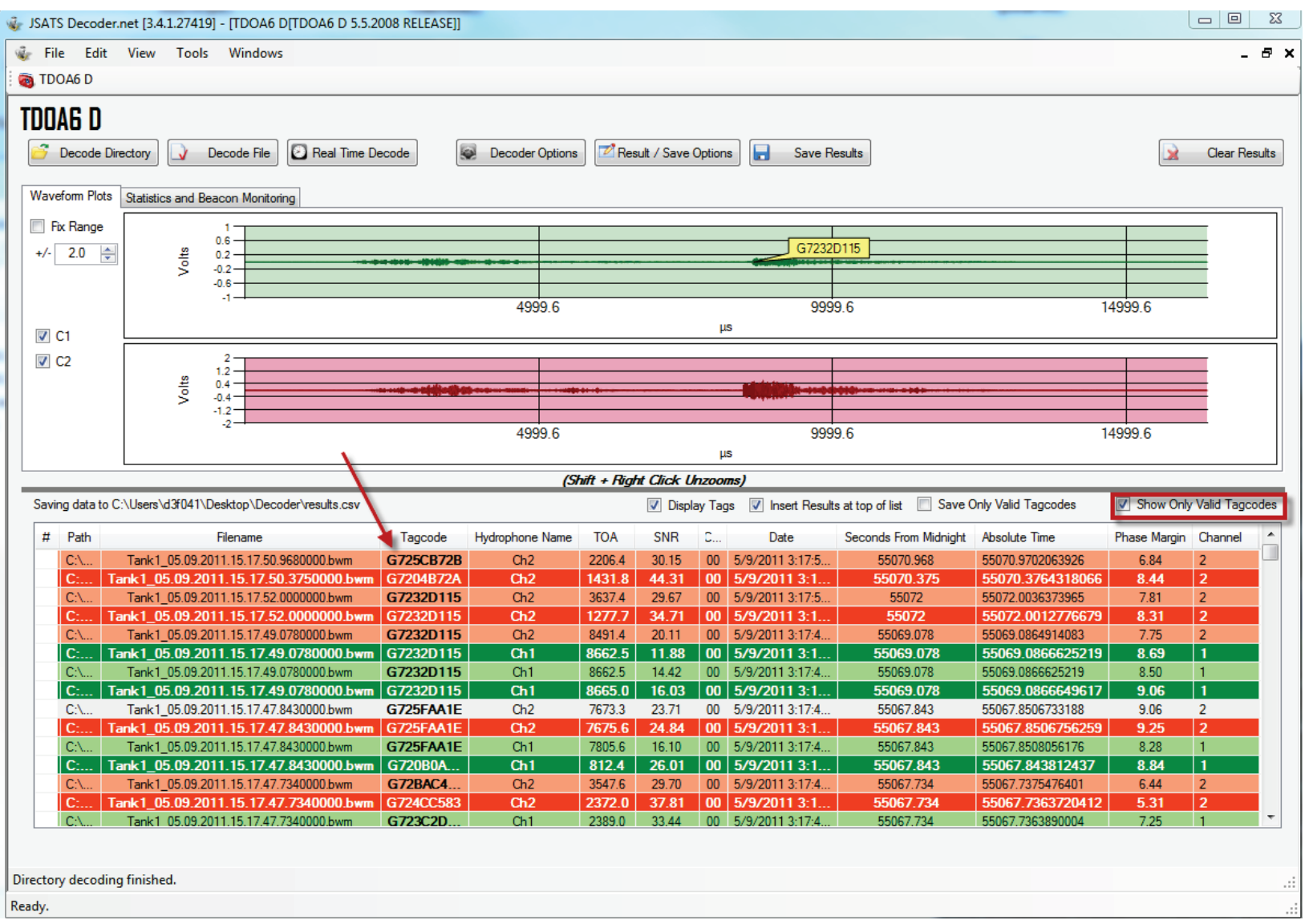

Figure 4.13. Tag Code Values with a G Prefix (identified by the red arrow) Are Displayed when the Show Only Valid Tagcodes Option is Selected (highlighted in red)

\subsubsection{Insert Results at Top of List Option}

When the Insert Results at Top of List box (Figure 4.12) is checked, the list of tag codes is increased by addition of new codes at the top of the spreadsheet. When this box is not checked, new tag codes are appended at the bottom of the spreadsheet.

\subsubsection{Display Tags Option}

The decoder program consumes memory and central processing unit (CPU) cycles to display tag codes. If tag codes are not displayed, decoding can be completed approximately $20 \mathrm{~ms}$ more quickly per file. While not a significant time cost for a single code, the time required to display thousands of tag codes contained in the data directory can be substantial. Consequently, it is best practice not to check the Display Tags box (Figure 4.12) when performing real-time decoding in potentially noisy environments.

\subsection{Clear Results}

The Clear Results button (highlighted in Figure 4.14), when clicked, will remove data from the results window. 


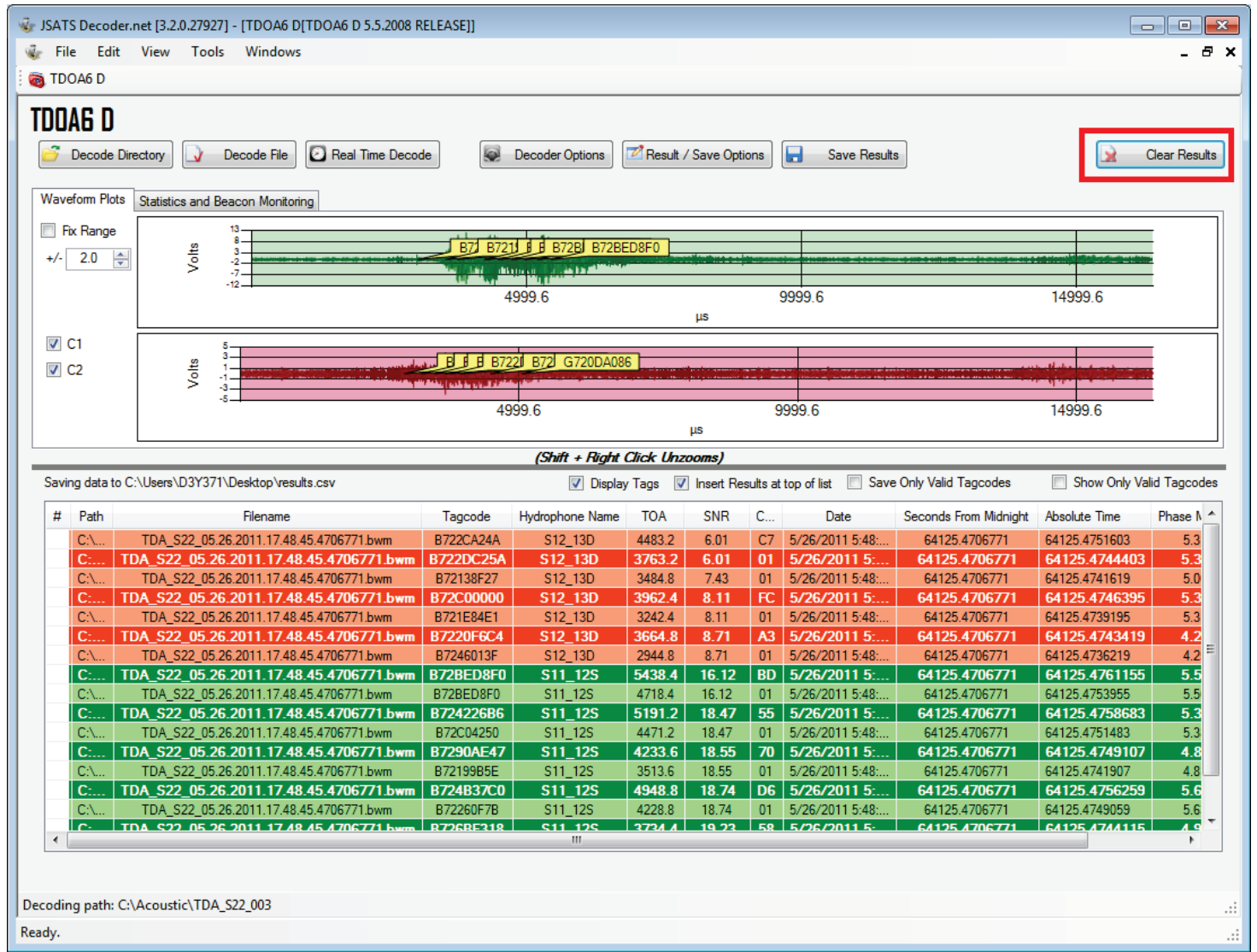

Figure 4.14. Clear Results Option (highlighted in red)

If the decoder is decoding files and the Clear Results button is pressed a Confirm Data Clear alert box will appear (Figure 4.15).

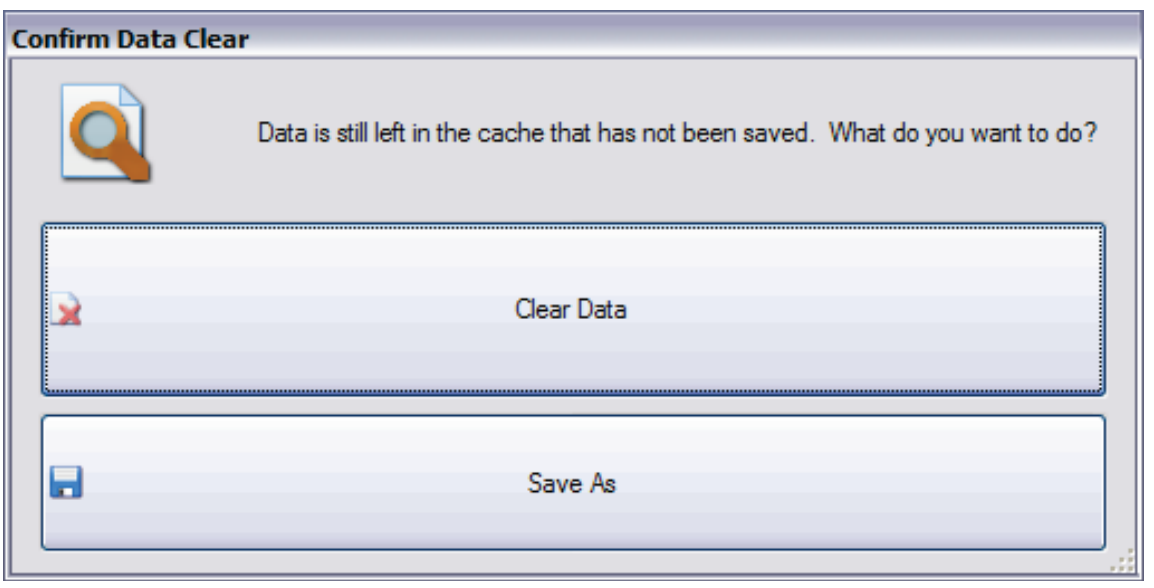

Figure 4.15. Confirm Data Clear Alert Box 


\subsection{Decoding}

Several methods for decoding waveform files are included with this software: decoding a single file, decoding directories, and real-time decoding. Each is described below.

\subsection{Decoding a Single File}

To decode a single file, press the Decode File button in the Decoder Command Bar (Figure 5.1).

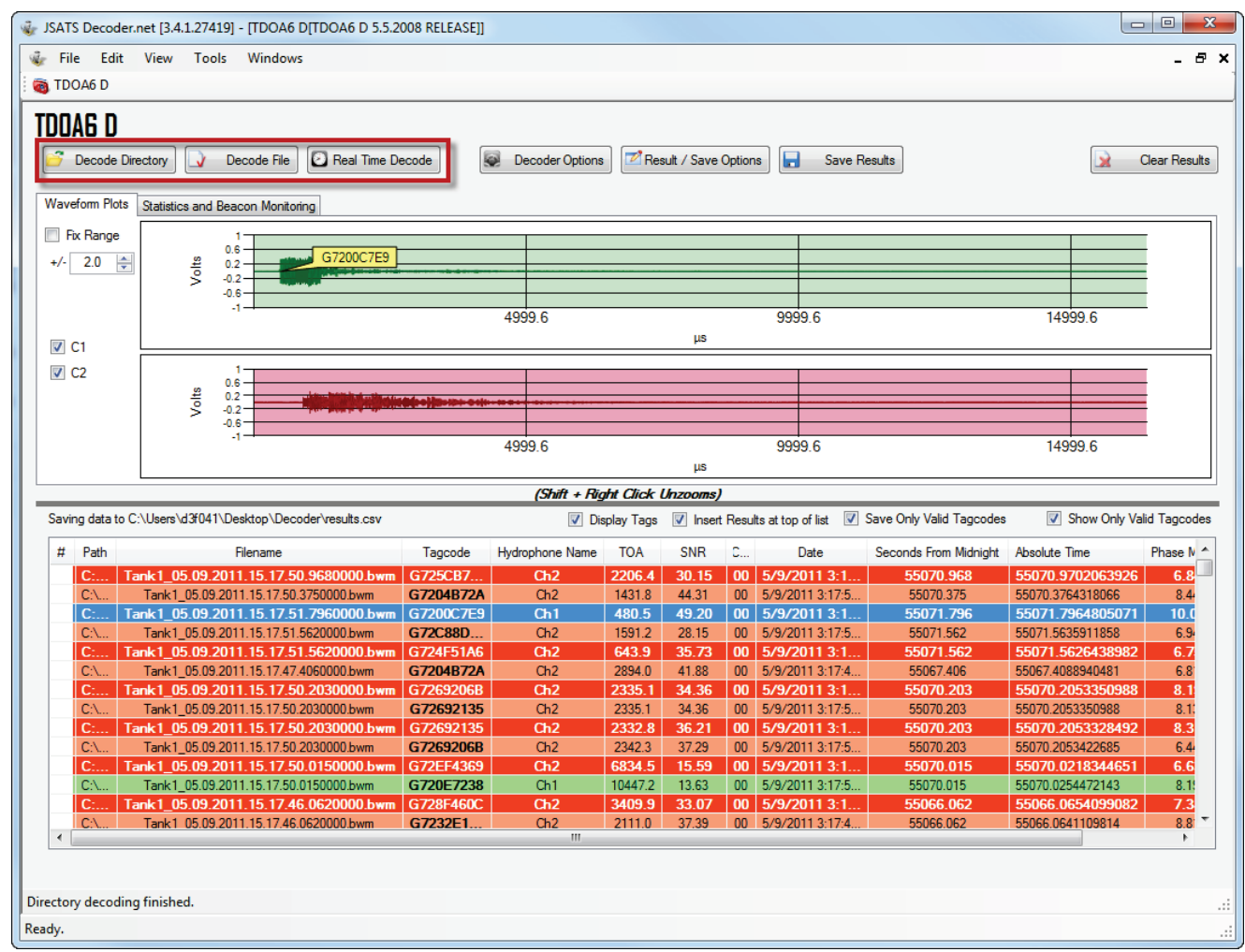

Figure 5.1. Decoder Command Bar Options (highlighted in red)

When this button is selected, a file dialog menu (Figure 5.2) will appear. 


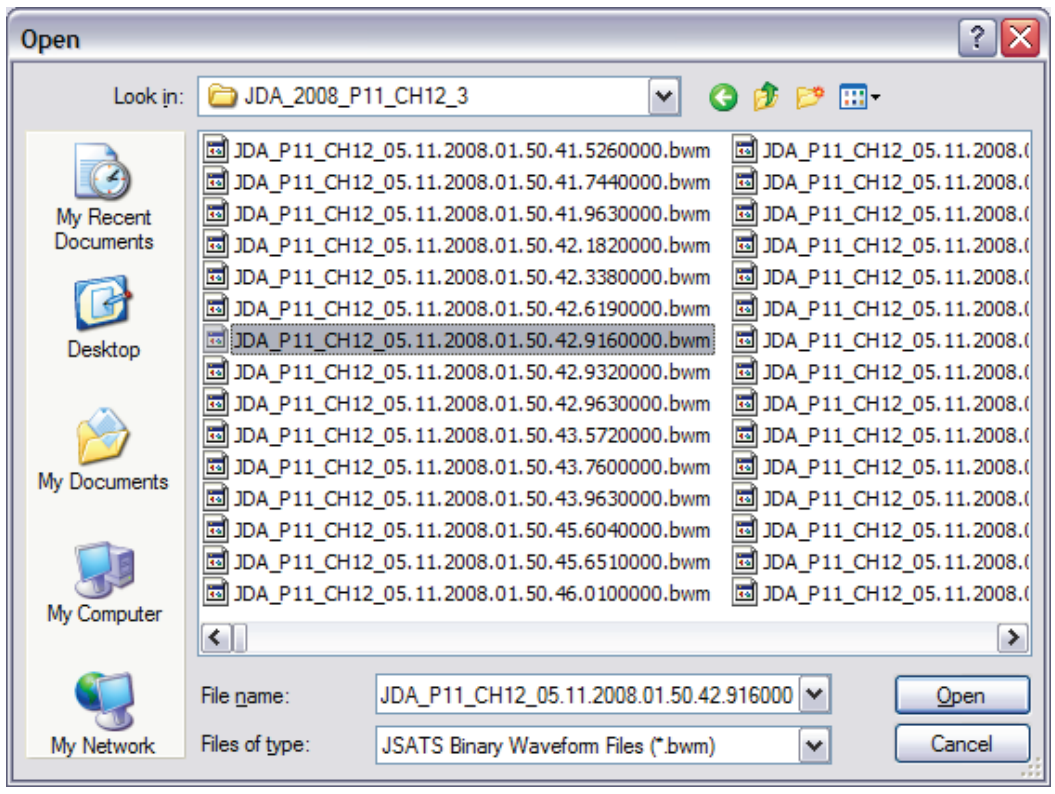

Figure 5.2. Decode File Open File Dialog Box

Select the file to be decoded or type the file name in the File name text box. Select Open to start the decode process or Cancel to terminate the Decode File operation.

\subsection{Decoding a Directory}

Post-processing of an existing file, directory, or series of directories, is initiated by selecting the Decode Directory button on the Decoder Command Bar (Figure 5.1).

When Decode Directory is selected, the Directory Options dialog box shown in Figure 5.3 will open, allowing data processing options to be selected. Select options and click Ok to initiate decoding of the data in a directory, or Cancel to exit the decoding process. 


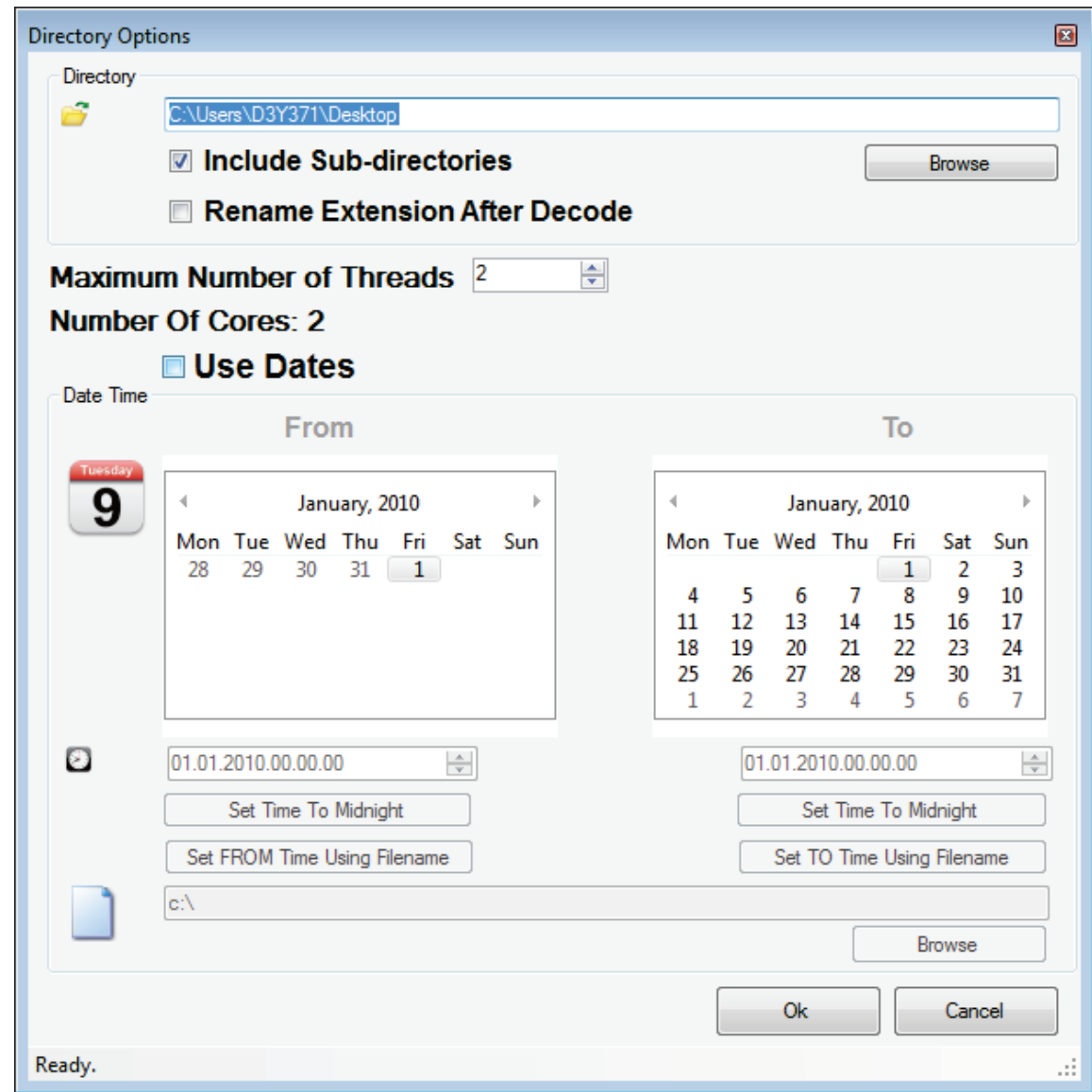

Figure 5.3. Decoder Directory Options Dialog Box

\subsubsection{Selecting the Decoding Directory}

The directory containing the data to be decoded is selected by entering the preferred name in the Directory text box (Figure 5.3) or by pressing the Browse button to locate the required folder. Selecting the Browse button opens the Browse for Folder dialog box (Figure 5.4). Navigate to the directory to be decoded, select the directory, and then click OK. Selecting Cancel will close the dialog box and no changes will appear in the Decode Directory Options dialog box. 


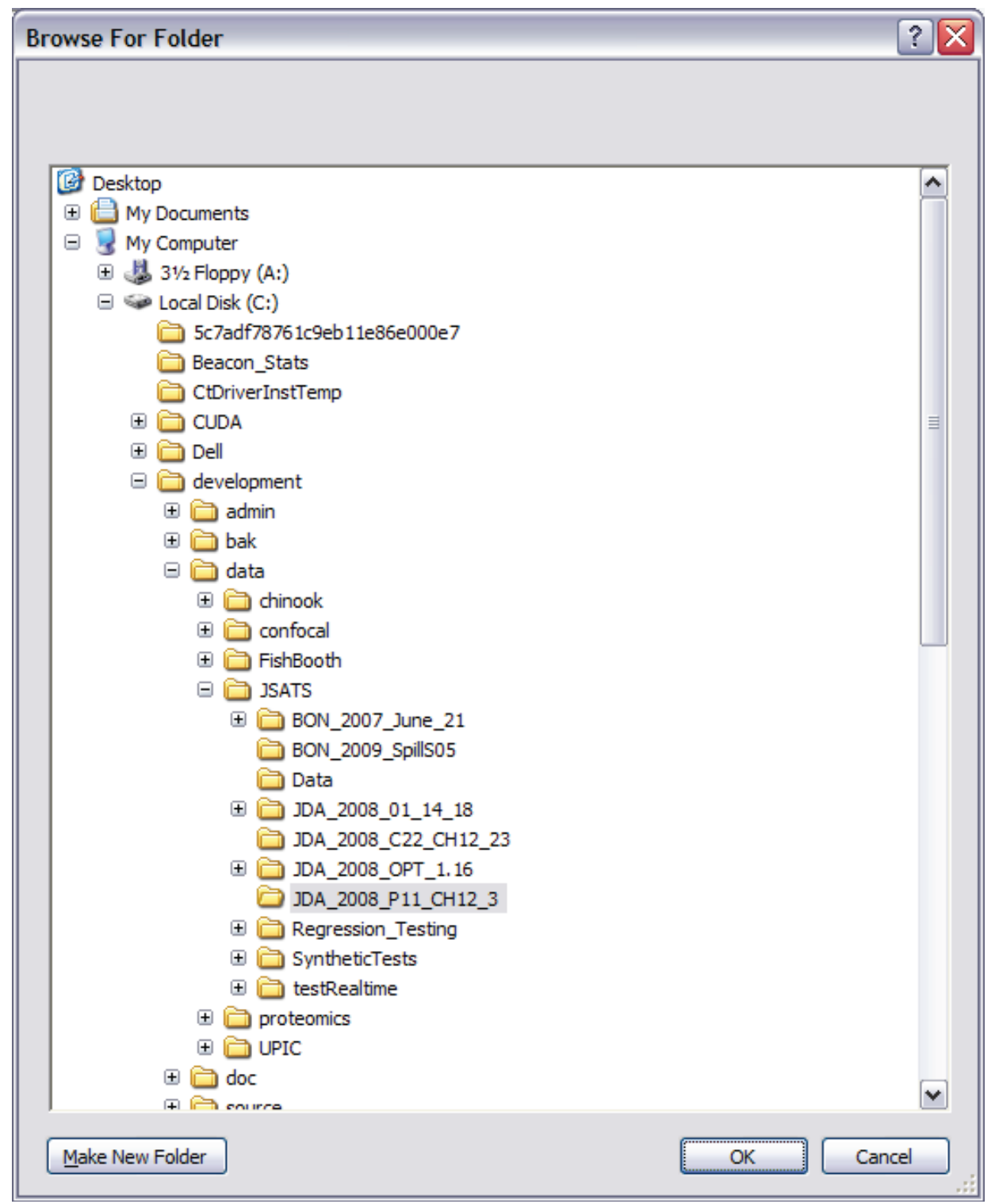

Figure 5.4. Decode Directory Browse for Folder Dialog Box

\subsubsection{Include Sub-Directories Option}

To exclude the decoding of sub-directories, i.e., only decode the top-level of a directory containing sub-folders, uncheck the Include Sub-directories box (Figure 5.3). Otherwise, the decoder will process files found in the directory specified as well as all sub-directories within the directory.

\subsubsection{Rename Extension After Decode Option}

Checking the Rename Extension After Decode box (Figure 5.3) will instruct the decoder to rename the extension of decoded files after decoding is complete. This is useful for verifying whether data have been processed. 


\subsubsection{Use Dates Option}

The Use Dates command (Figure 5.3) allows the file dates to be chosen for files to be decoded. This is useful when reprocessing data or subsampling the data for a given time period, because the directories are chosen by date and don't have to be identified by name. To use this option complete the following steps:

1. Check the Use Dates box.

2. Select the start date in the calendar displayed in the From dialog box.

3. Select the end date in the calendar displayed in the To dialog box.

Respective From and To dates can also be entered in the numeric spin boxes below the calendars. Times are set in the following format: Month.Day.Year.Hour.Minute.Second. Values can be reset within the day selected in the calendar by pressing the Set Time To Midnight button.

Because the detector saves files with the date in the name during data acquisition, you can select the Set FROM Time Using Filename or Set TO Time Using Filename buttons as shown in Figure 5.5.

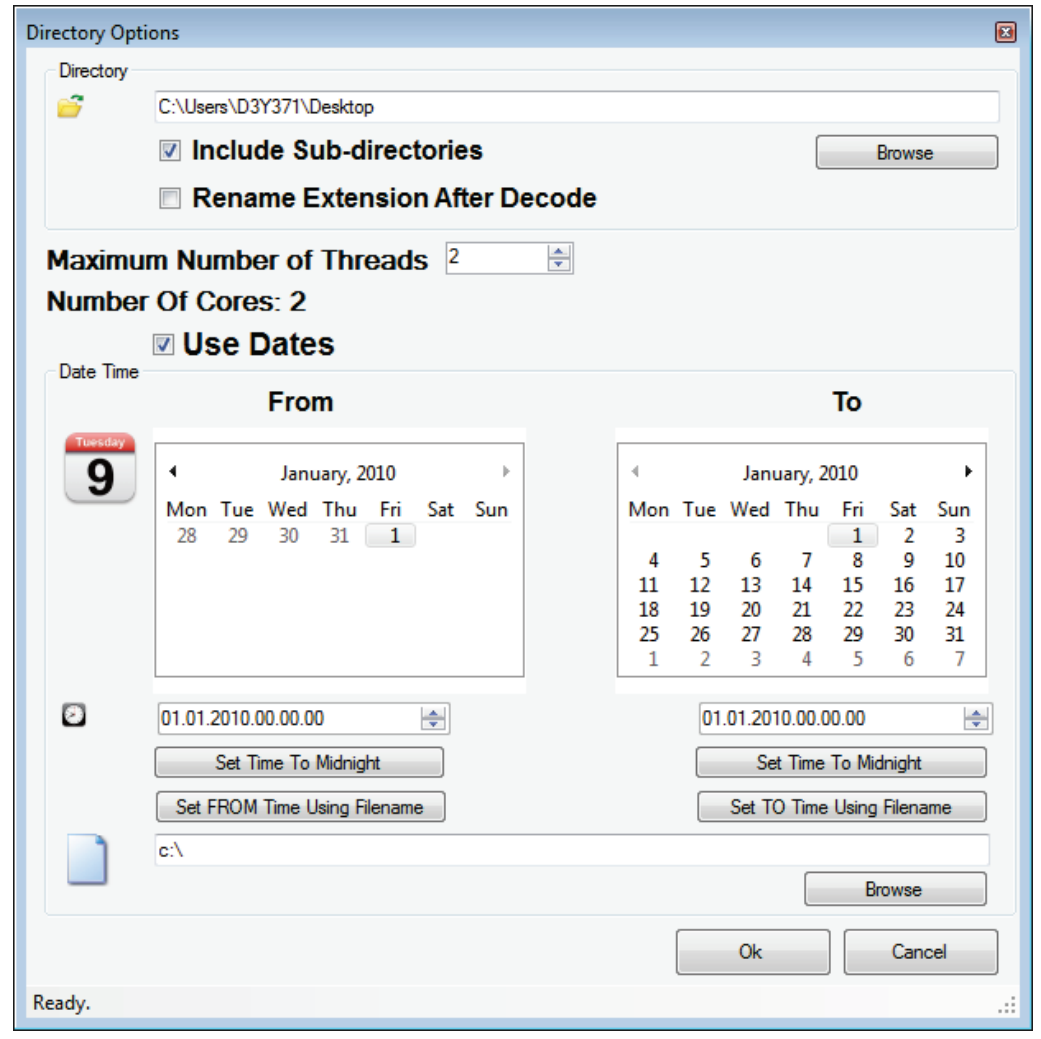

Figure 5.5. Decoder Directory Selection Options

The name of the file was entered in the spin box for the example shown in Figure 5.5. When selecting the Browse option to choose a file, the Open file dialog box (Figure 5.6) will appear, allowing navigation to the required file. 


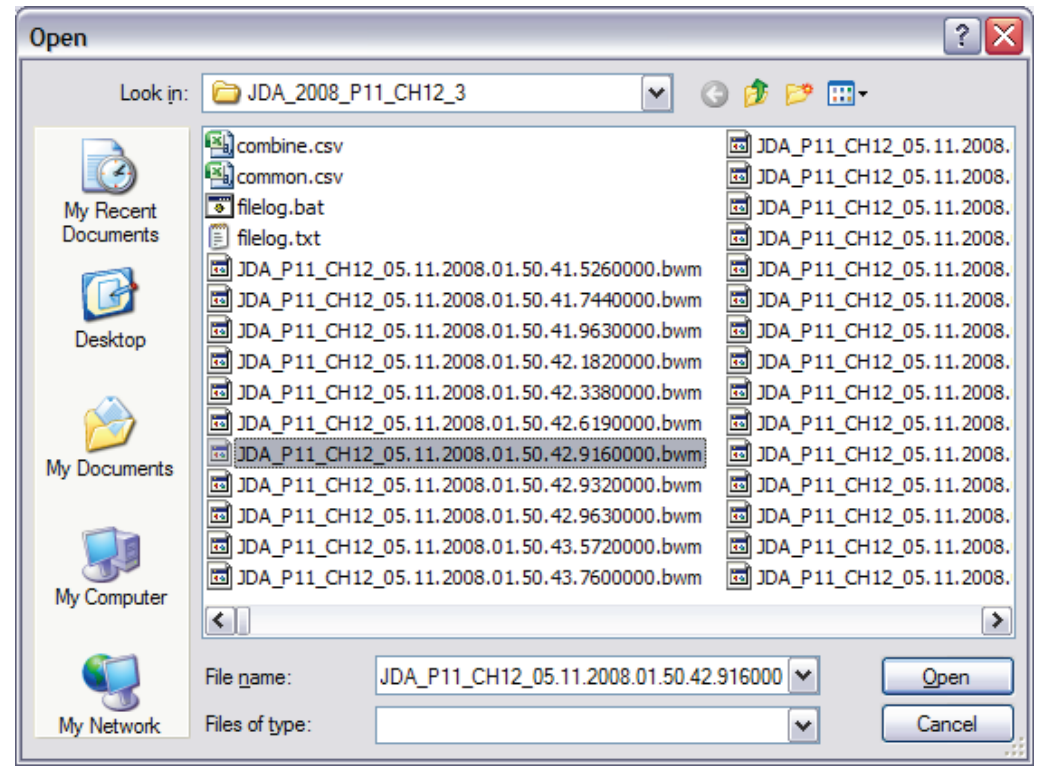

Figure 5.6. Selecting the Decode Start Date and Time from a .bwm File

When the file with the desired start date and time is located, highlight it, select Open, and press the Set FROM Time Using Filename button (Figure 5.7) to complete selection of a start date and time. When Set FROM Time Using Filename is selected, the date in the From calendar will change to the selected file name. This process can be repeated to select a file to identify the TO date to complete identification of the time period over which processing will occur.

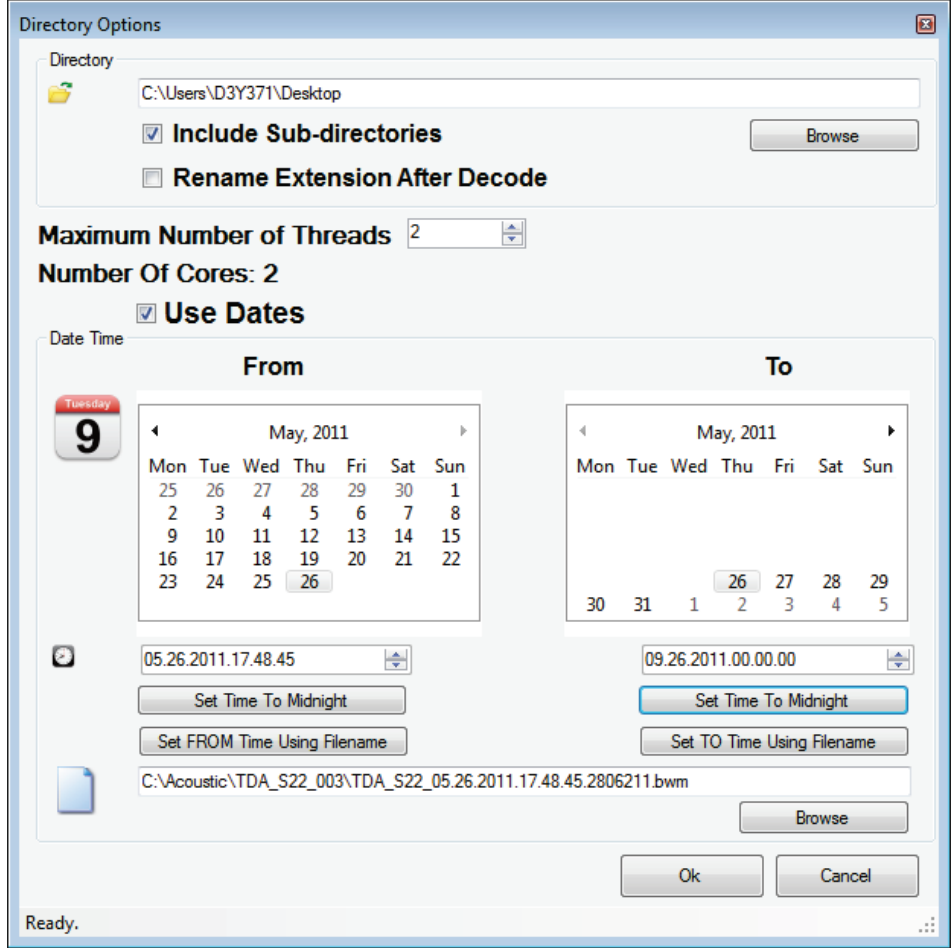

Figure 5.7. Updating the Calendar Using Set From and Set To Times 


\subsubsection{Maximum Number of Threads}

The Maximum Number of Threads field (Figure 5.7) can be adjusted to increase or decrease the number of instances of the decoder running at any one time. If the number of threads is the same as the number of cores on the decoding computers' CPU, the application decodes that number of files simultaneously, allowing the application to decode files more quickly. If the number of threads exceeds the number of cores, the application will attempt to decode as many files simultaneously as possible.

As a rule of thumb, set the maximum number of threads to twice the number of cores available in the processing computer if there is no other application that needs to run. If another application will be running at the same time as the JSATS decoder and needs to execute as quickly as possible, such as the JSATS detector during real-time decoding (explained in Section 5.3), reduce the maximum number of threads to less than the number of cores.

\subsubsection{How Do I Know It Is Decoding?}

When the decoder directory options are configured in the Directory Options dialog box, press the Ok button (Figure 5.8).

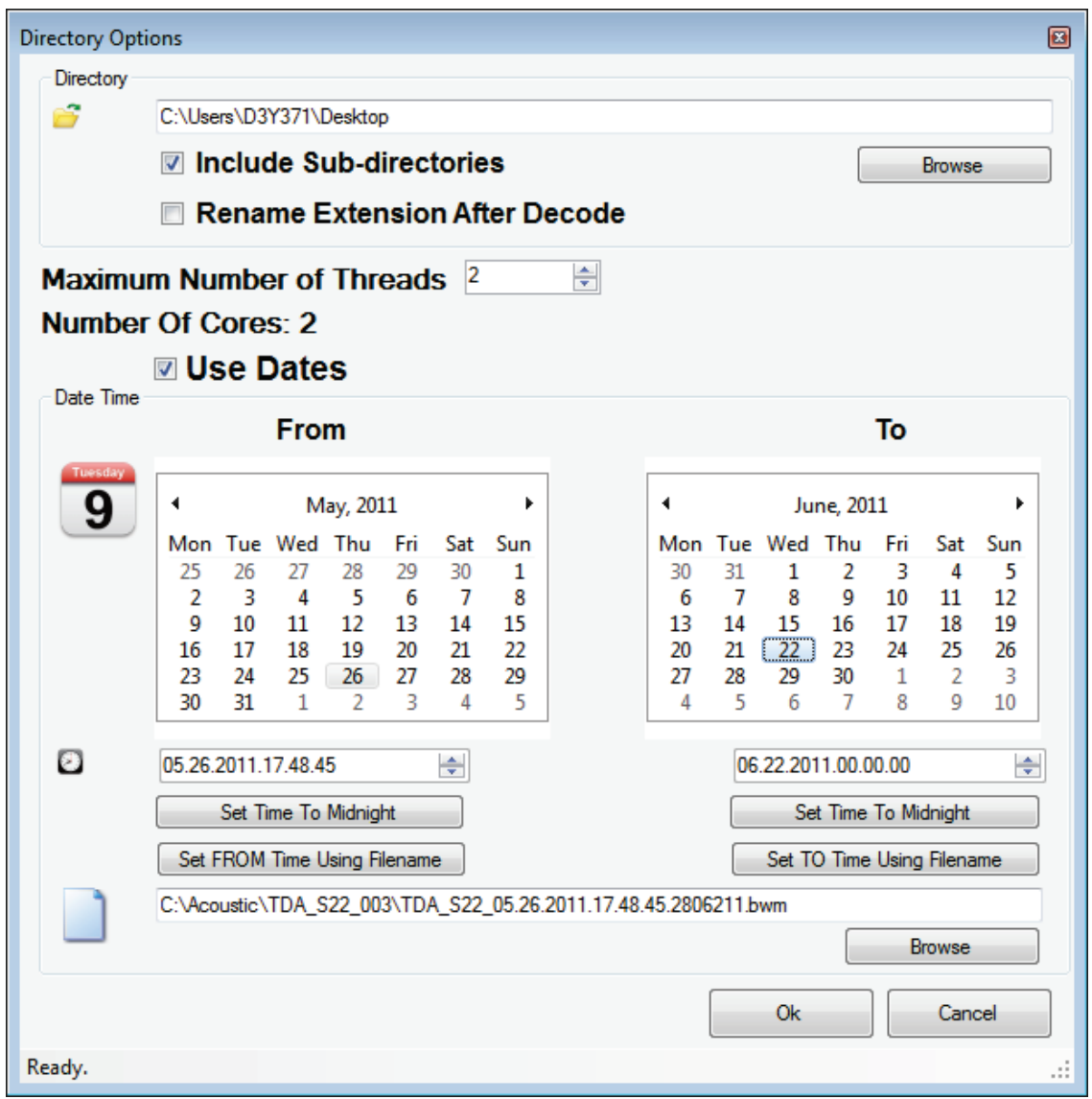

Figure 5.8. Select Ok to Start the Decoding Process 
The decoder Directory Options dialog box will disappear and the main window shown in Figure 5.9 will start displaying decoded data in the spreadsheet in the bottom frame of the window, indicating the decoding progression. The Decoder Command Bar (Decode Directory, Decode File, Real Time Decode), Decoder Options, and Save Command Bar (Result/Save Options and Save Results) buttons at the top of the Decoder window will be grayed out and will be inaccessible. A green progress bar at the bottom of the window will indicate the percentage of files processed. The list of processed files will be continuously appended as the decoding progresses.

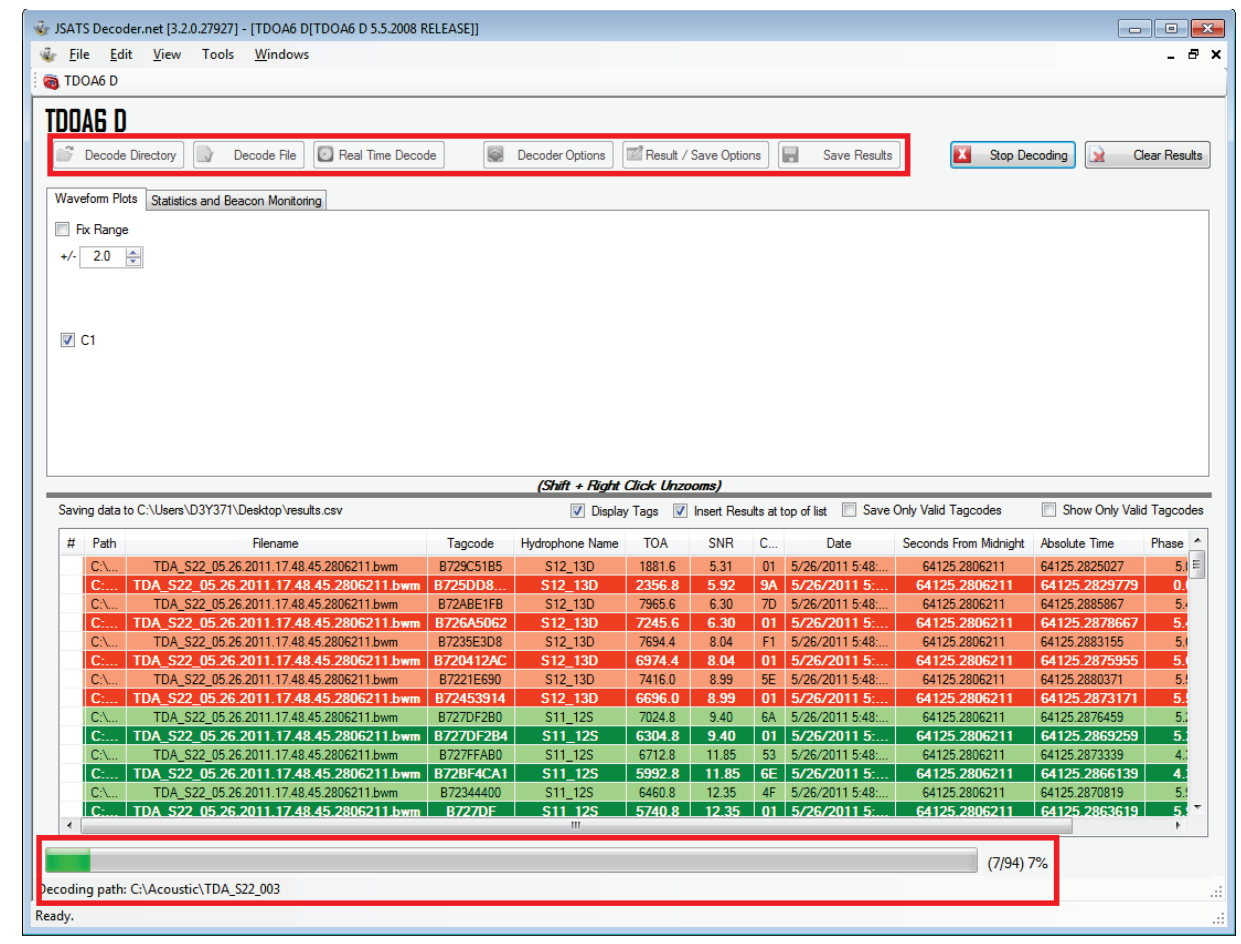

Figure 5.9. Options at the Top of the Decoder Window (highlighted in red) Are Disabled during the Directory Decoding Process. The progress bar at the bottom of the window (also highlighted in red) indicates processing advancement.

\subsubsection{Stopping the Directory Decoding Process}

To stop data decoding within a directory after it has begun select the Stop Decoding button (highlighted in Figure 5.10). The results from files that have been processed will be saved automatically to the Results file. 


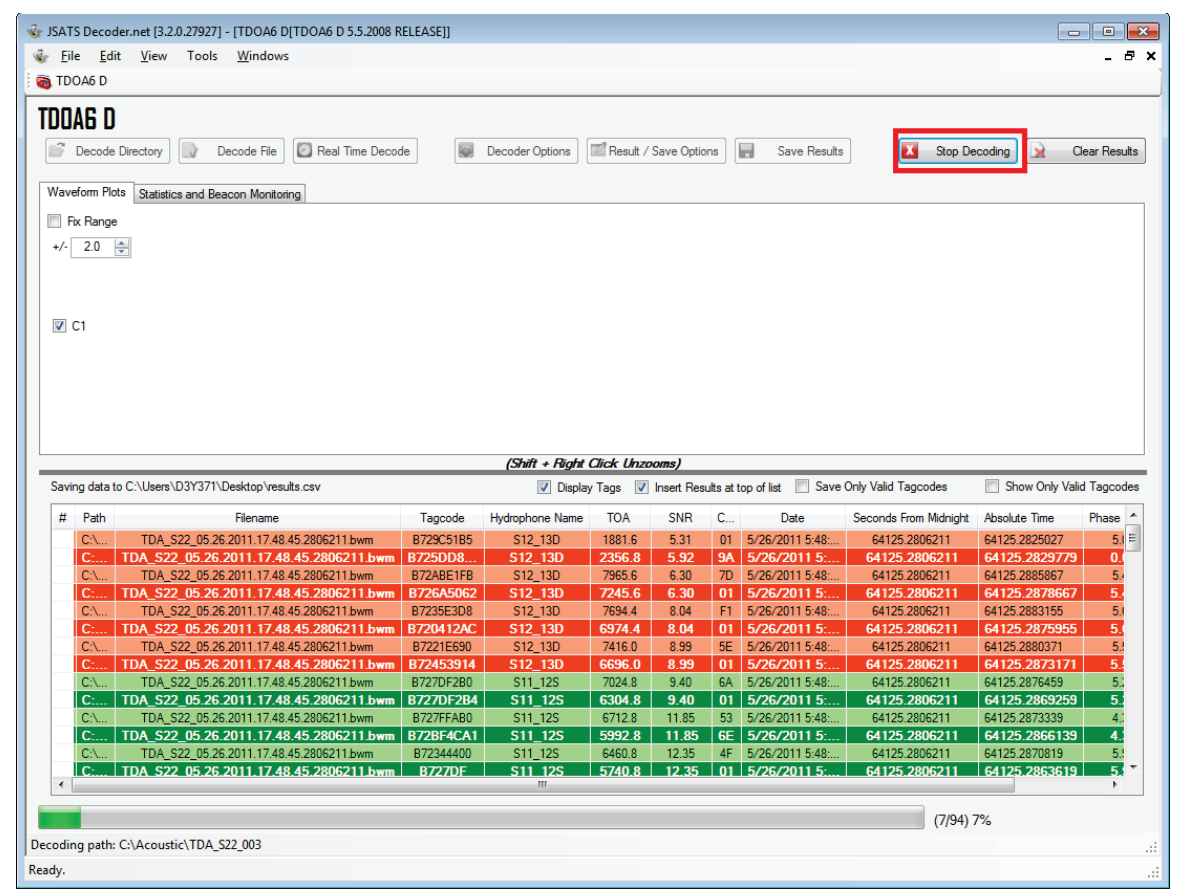

Figure 5.10. Select the Stop Decoding Button to Terminate a Directory Decode

\subsection{Real-Time Decoding}

Real-time decoding is accomplished when the JSATS detector and decoder are running simultaneously. When the JSATS detector is running and collecting files, the decoder can automatically access and decode the new waveform files created by the detector. This assists in managing the number of waveform files saved because post-processing will not be required and waveform files can be decoded as they are acquired, then discarded. However, if retaining waveform files after decoding is required for future investigation, the decoder can rename the waveform file names extension and save the processed files.

\subsubsection{Real-Time Decoding}

Real-time decoding is customized and launched by selecting the Real Time Decode button (highlighted in Figure 5.11). 


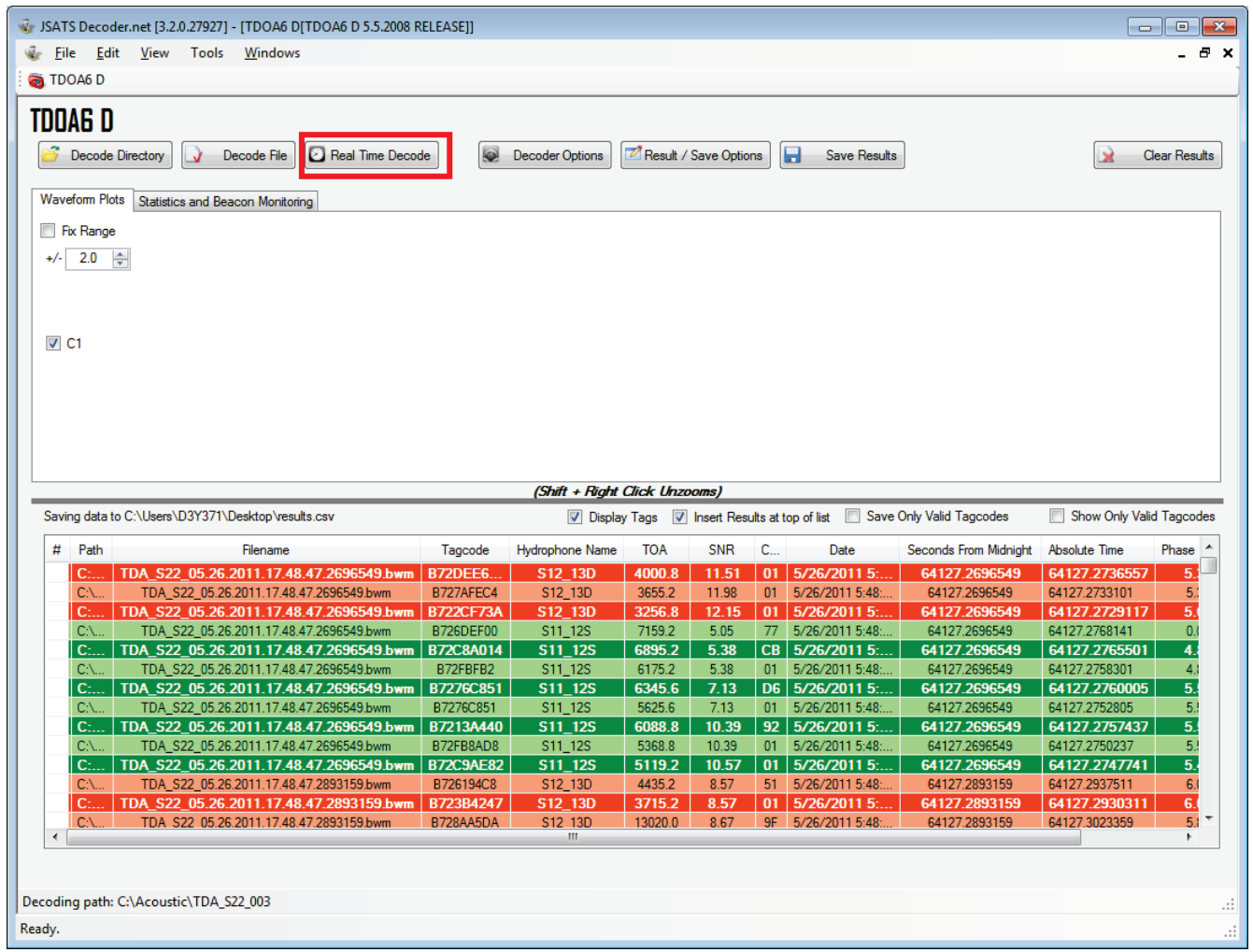

Figure 5.11. Real-Time Decoding Option (highlighted in red)

When the Real Time Decode button is selected, the Real-Time Decoder Options dialog box shown in Figure 5.12 opens.

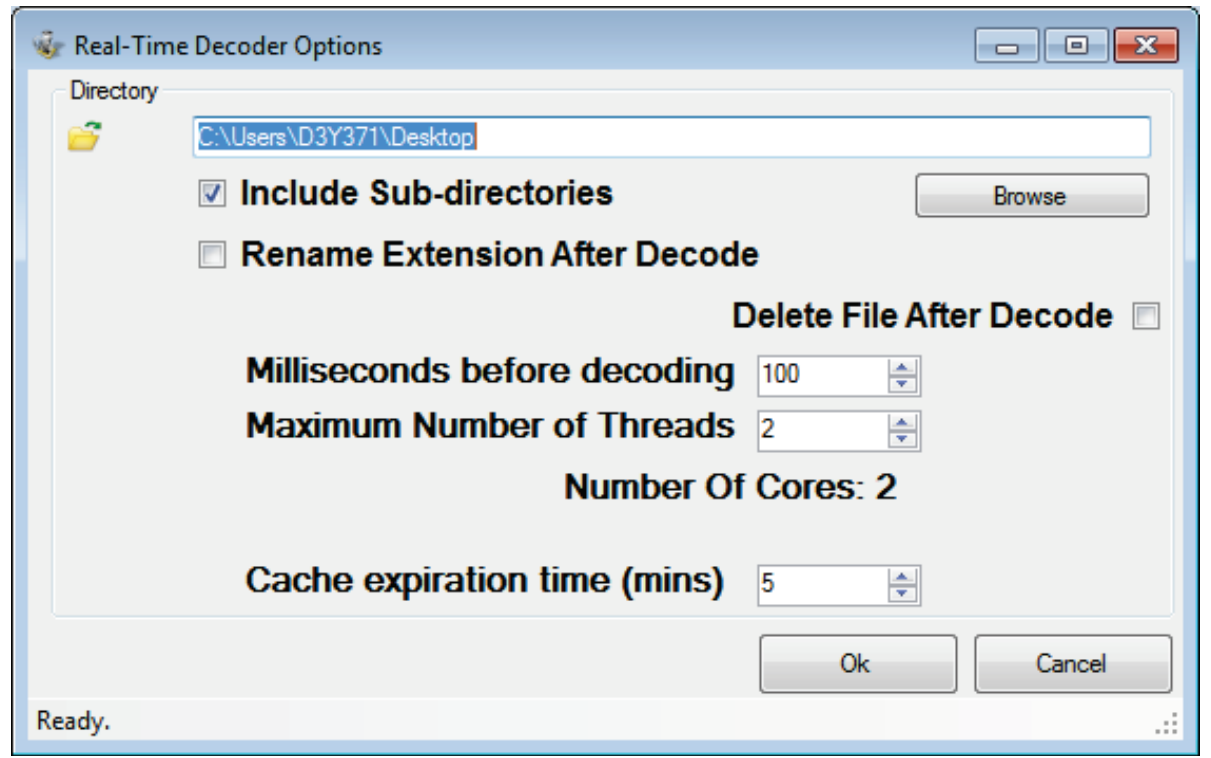

Figure 5.12. Real-Time Decoder Options Dialog Box 
Enter the name of the directory where the JSATS detector has been directed to save data into the Directory text box (Figure 5.13). Confirm that you are decoding candidate tag waveforms from only one receiver board with the JSATS decoder. Multiple copies of the JSATS decoder can be run to real-time process waveform files from multiple receiver boards simultaneously if enough cores are available on the CPU to handle the additional processing. A multi-core CPU or computer with multiple CPUs is necessary to operate real-time decoding during data acquisition.

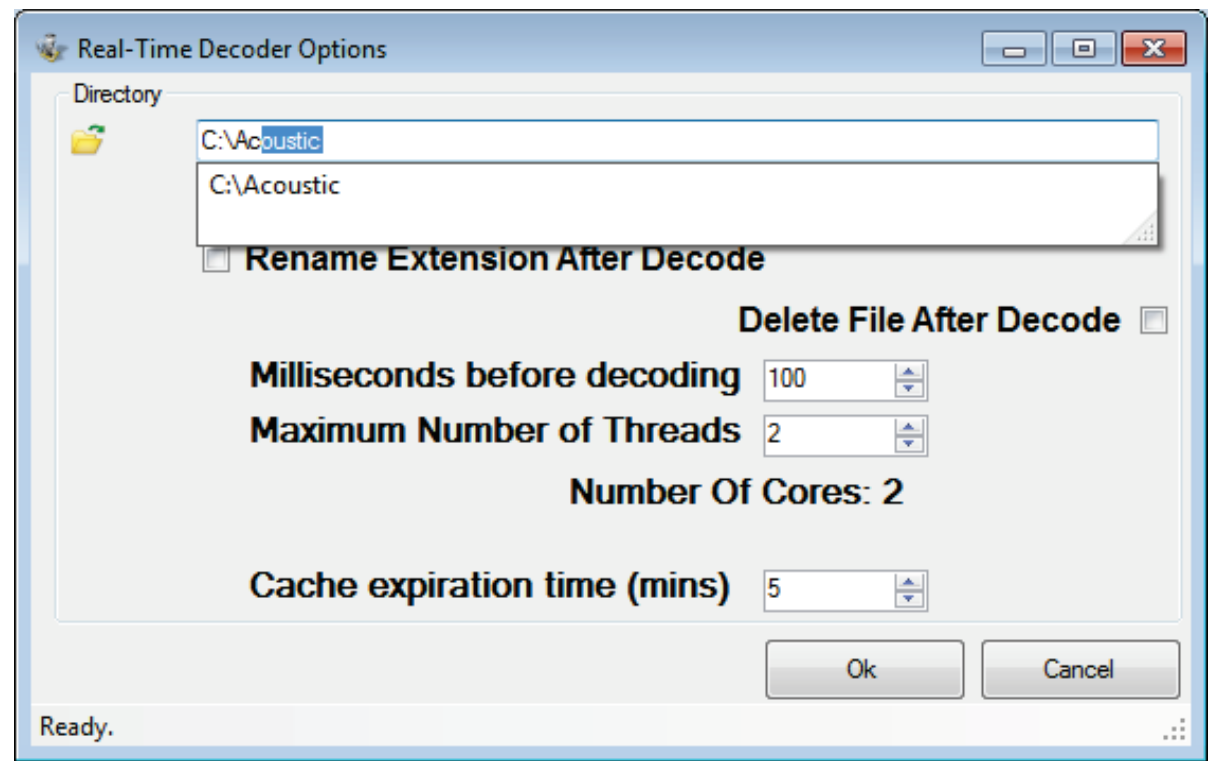

Figure 5.13. Real-Time Decoder Directory Selection

As a directory name is entered, the text box will suggest directory names that are available on the computer. The directory may also be located by pressing the Browse button and navigating to the directory in the Browse For Folder dialog box (Figure 5.14). 


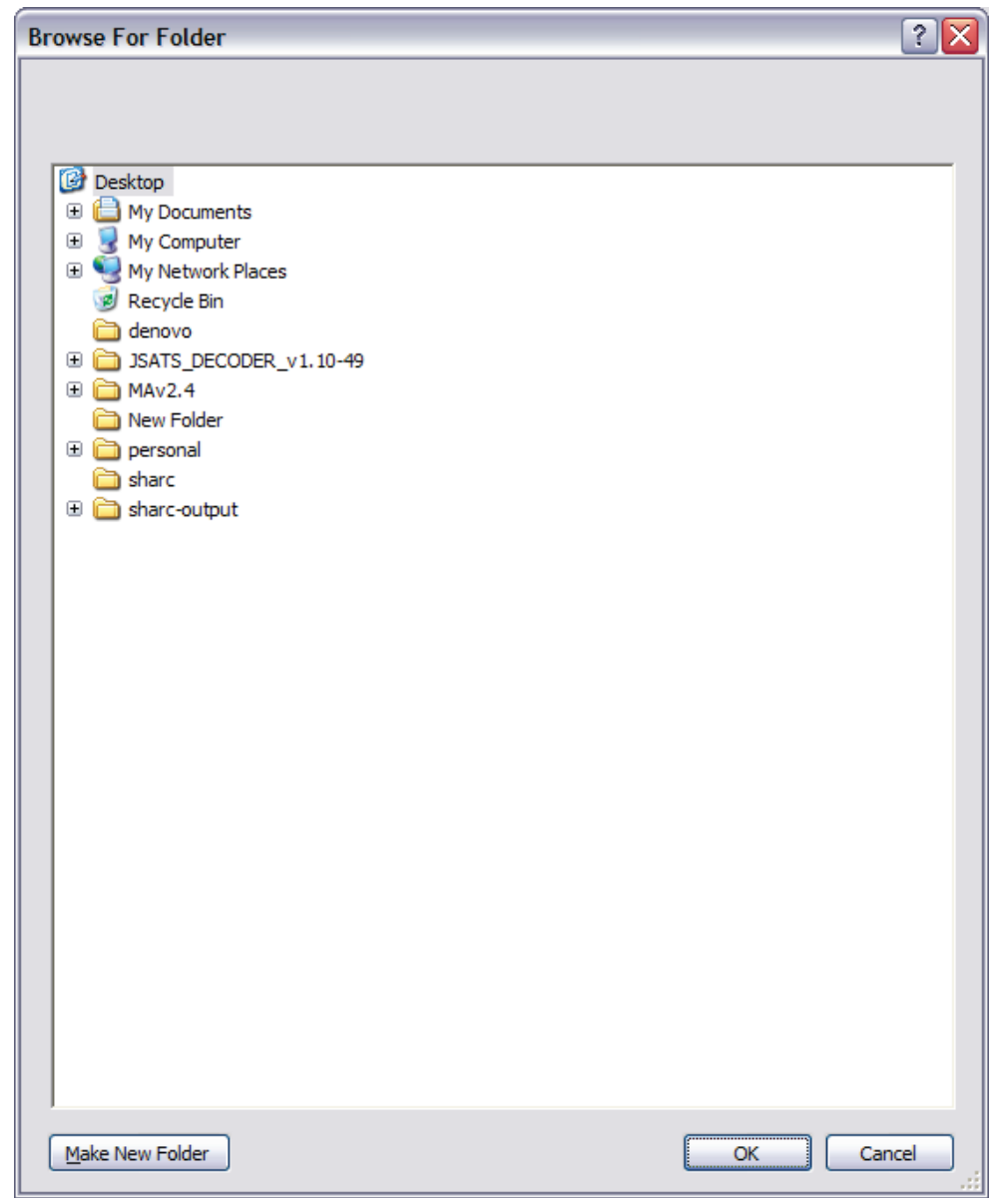

Figure 5.14. Real-Time Decode Directory Browse for Folder Option Dialog Box

\subsubsection{Include Sub-Directories}

Real-time decoding can be achieved with a single directory or, if the Include Sub-directories option is selected, a directory and its sub-directories (Figure 5.12). It is recommended that the Include Subdirectories option always be selected.

\subsubsection{Rename Extension After Decode}

The Rename Extension After Decode option (Figure 5.15) will rename decoded files with a .com extension, by default. When this option is selected, the renamed files will be saved and the Delete File After Decode box will be inaccessible (Section 5.3.4). The Rename Extension After Decode feature is very useful if all waveforms are being saved, including those that have been decoded, and the decoder cannot keep up with the number of incoming waveform files. If the number of candidate tag waveforms to be processed by the decoder overwhelms the real-time feature of the decoder, the user can post-process the remaining files later using the Decode Directory option (see Section 5.2). The decoder will only decode files that have the.$b w m$ extension; it will ignore files that have other extension designations. 


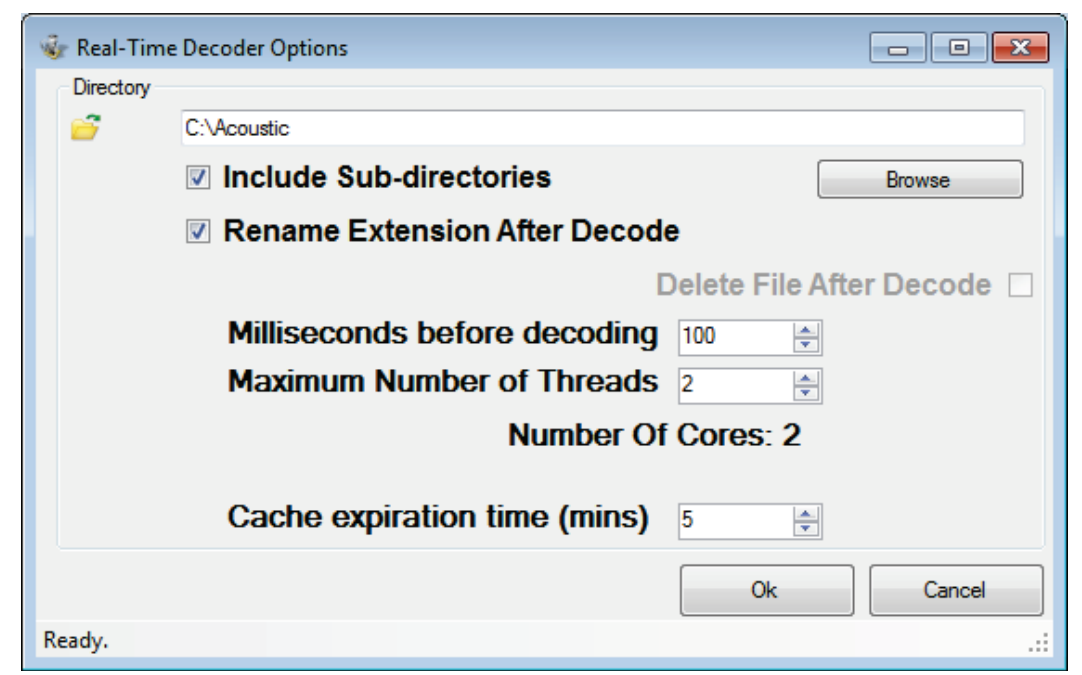

Figure 5.15. Real-Time Decoder Options Dialog Box

The extension of the renamed file can be changed from .com to any extension if the file "JSATSDecoder.net.exe.config" file is modified with a text editor (e.g., Notepad or WordPad). This configuration file can be found in the directory in which the JSATS decoder program was installed on the computer, usually the JSATS Decoder Program Files directory (Figure 5.16). Near the bottom of the file, there is a RenameExtension setting. Within the two $<$ value $></$ value $>$ tags, the extension can be changed from .com to the desired extension, such as .cmp (Figure 5.17).

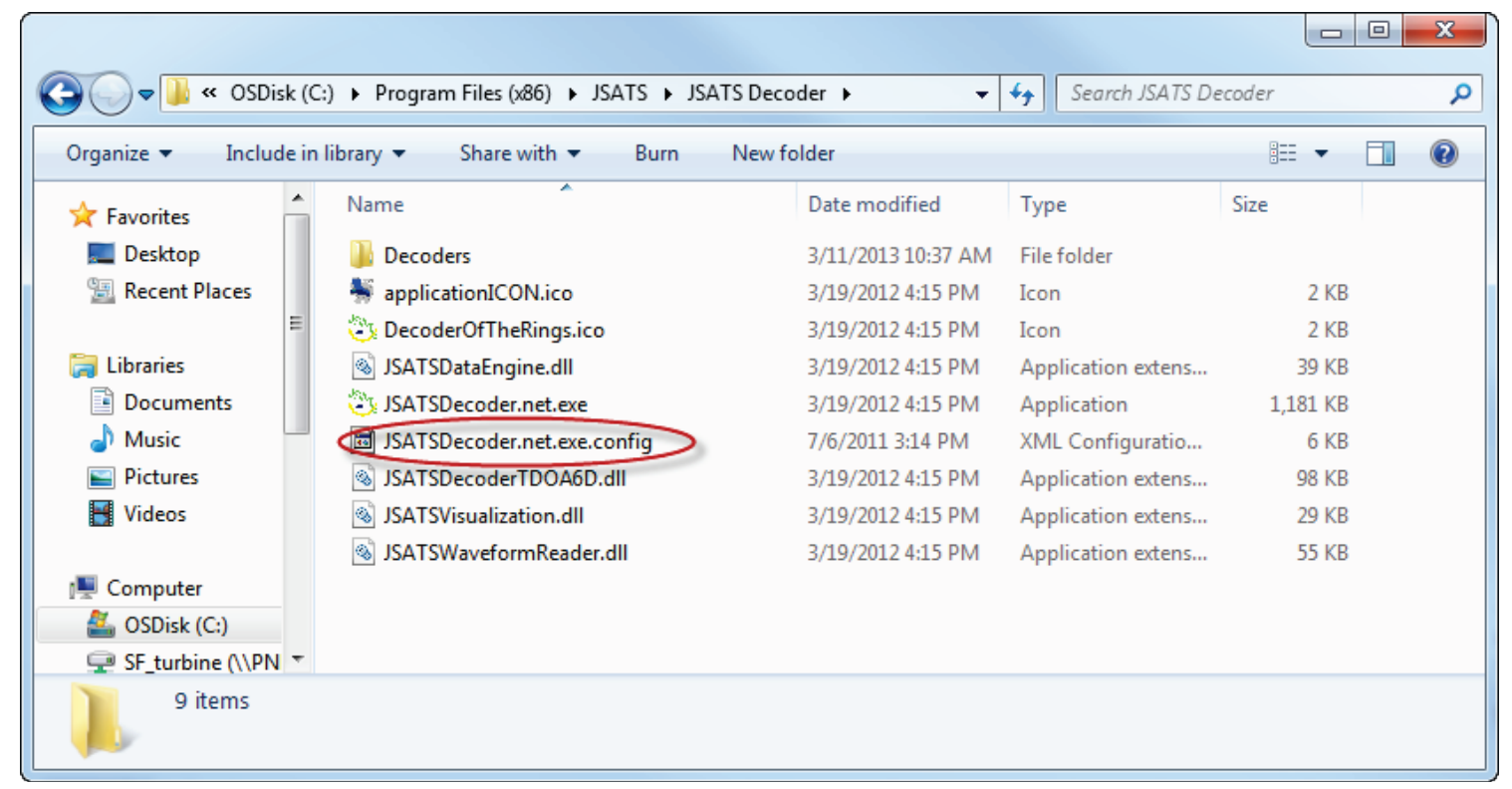

Figure 5.16. The JSATS Decoder Configuration File Is Commonly Located in the Program Files Directory 


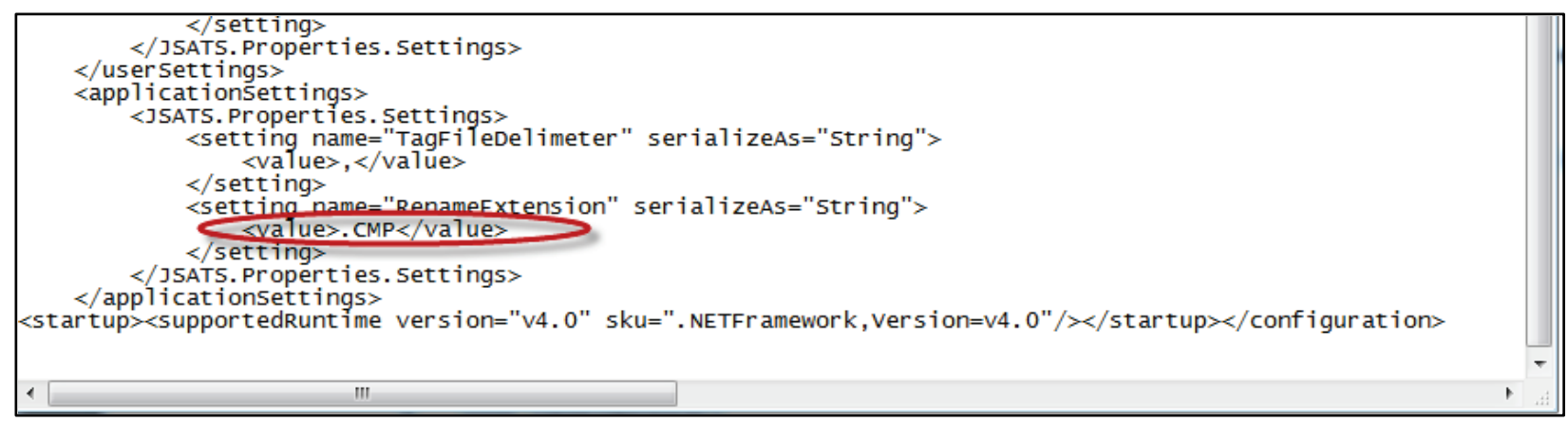

Figure 5.17. The File Extension for Renamed Files Can Be Changed by Modifying the Configuration File

\subsubsection{Delete File After Decode}

Selecting the Delete File After Decode checkbox (Figure 5.15) will result in waveform files to be deleted after they have been decoded. As a precautionary measure, this option is not set by default to avoid deleting files until all aspects of data flow through the JSATS receiving system have been verified to be functioning as intended.

\subsubsection{Milliseconds Before Decoding}

The decoder will pause for a few milliseconds prior to decoding a newly saved file, allowing the detector to finish writing the file to disk. The recommended wait time is $100 \mathrm{~ms}$ - the default value in the Milliseconds before decoding spin box found in the Real-Time Decoder Options dialog box (Figure 5.15). If the wait time is too short, the decoder will attempt to read the waveform before the detector has completed writing the file to disk and the system will crash.

\subsubsection{Maximum Number of Threads}

Selection of the Maximum Number of Threads in the Real-Time Decoder Options dialog box (Figure 5.15) has the same effect as the selection of the maximum number of threads in the Decode Directory dialog box (Section 5.2.5).

The Maximum Number of Threads option allows the user to increase or decrease the number of instances of the decoder running at any one time. If the number of threads is the same as the number of cores, the application will decode that number of files simultaneously, allowing the application to decode files more quickly. If the number of threads exceeds the number of cores, the application will attempt to decode as many files simultaneously as possible.

When using real-time decoding, it is best practice to increase this field to as high as possible without affecting other applications. The maximum value of the field is twice the number of CPU cores. When at the maximum, the JSATS application will consume $100 \%$ of the CPU cycles available if files are being detected at a high rate. 


\subsubsection{Cache Expiration Time (mins)}

The Cache expiration time (mins) option (Figure 5.15), found in the Real-Time Decoder Options dialog box, is the maximum amount of time the application will store tag codes in the buffer memory before writing them to file. For example, if the cache limit is 1000 , and the expiration time is 30 minutes; when the decoder collects 200 tag codes in 30 minutes, it will write all 200 to the results .csv file. If it collects 1000 tag codes in 15 minutes, with the same expiration time, all 1000 will be written to the results .csv file after 15 minutes. This feature is useful in quiet environments or other circumstances when the number of candidate waveforms per unit time is low. 



\subsection{Beacon Monitoring}

This section describes an advanced decoder feature - beacon monitoring. This option monitors the codes transmitted by beacons deployed in the study area. Receiving and monitoring beacon transmissions assists in verifying the operation of hydrophones in a JSATS receiving array and are used to estimate detection range and detection efficiency.

\subsection{Statistics and Beacon Monitoring Window}

Select the Statistics and Beacon Monitoring tab in the Decoder window to open the beacon monitoring options and related controls (Figure 6.1). Selections include Load Beacon File, Save Beacon List, Add, Delete, Edit Selected Beacon, Stats Options, Delete Beacons, and Clear Statistics.

A list in the upper frame of the Decoder window displays beacons that were entered manually (see Section 6.2), beacons added using the context menu option (Section 4.2.3), or beacons added by file selection (see Section 6.6). Figure 6.1 shows three beacons in the beacon list.

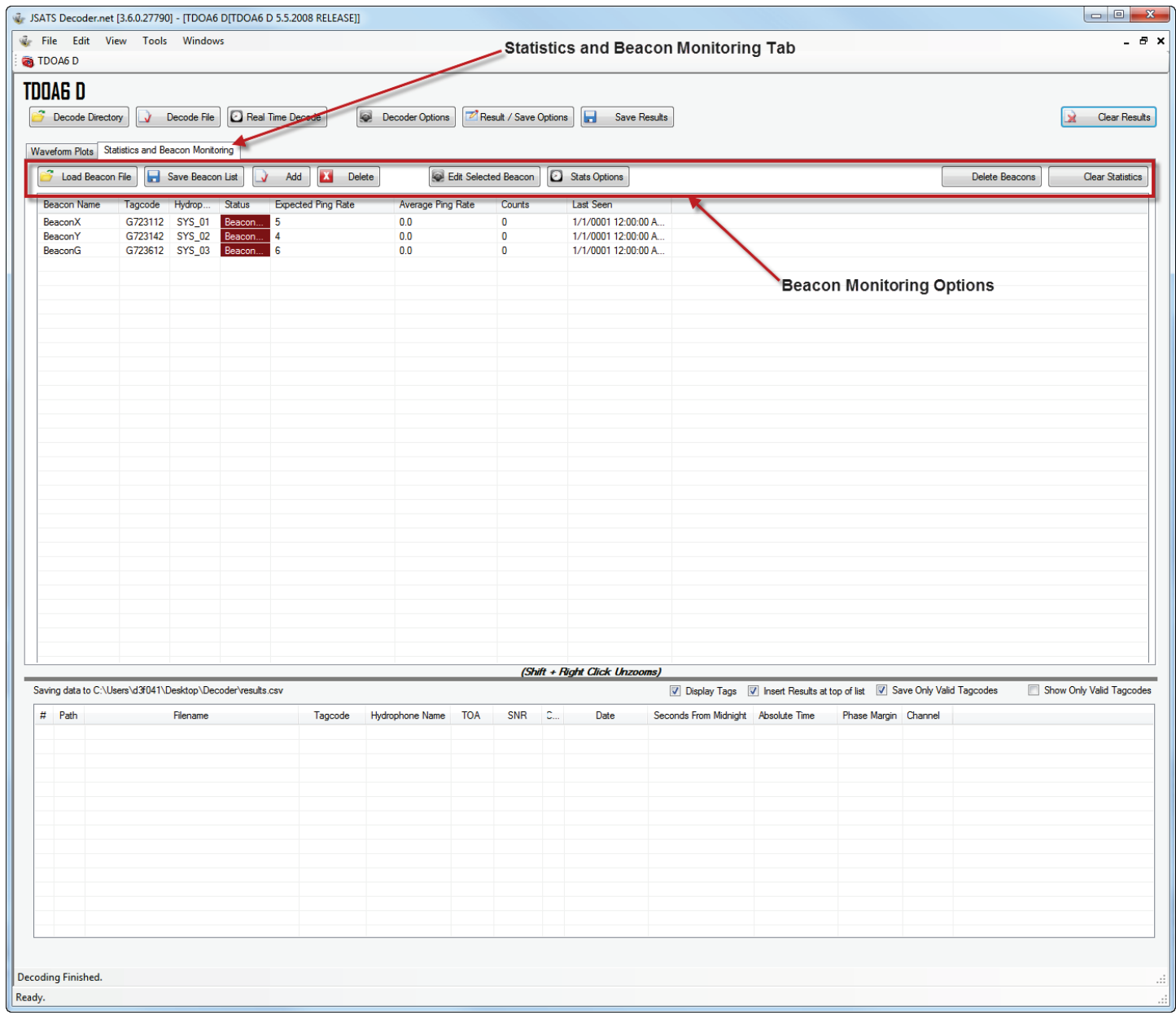

Figure 6.1. Statistics and Beacon Monitoring Options 
The beacon information contained in the spreadsheet includes the following:

- Beacon Name - an alias assigned to the beacon by the program user

- Tagcode - the code transmitted by the beacon, the code must include a $\mathrm{G}$ at the beginning of the tag code

- Hydrophone Name - the alias for the hydrophone receiving the beacon code (assigned in the Detector software)

- Status - decoder assessment of the operating status of the beacon

- Expected Ping Rate - the number of transmissions per unit time by the beacon

- Average Ping Rate - the number of transmissions per unit time observed for the beacon

- Counts - the number of times the beacon has been detected within a period of time

- Last Seen - the last time the beacon was detected.

\subsection{Adding a Beacon to the Statistics and Beacon Monitoring List}

To manually add a beacon, press the Add button in the Statistics and Beacon Monitoring window (Figure 6.1), which will open the Edit Beacon dialog box. Enter the beacon information in the Edit Beacon dialog box and press $\mathbf{O K}$ to accept the changes or the Cancel button to reject the changes (Figure 6.2). Tag codes can also be added to the beacon list by selecting a row(s) in the Results file, rightclicking to open the Context menu, and selecting Monitor Tagcode from the options (Section 4.2.3).

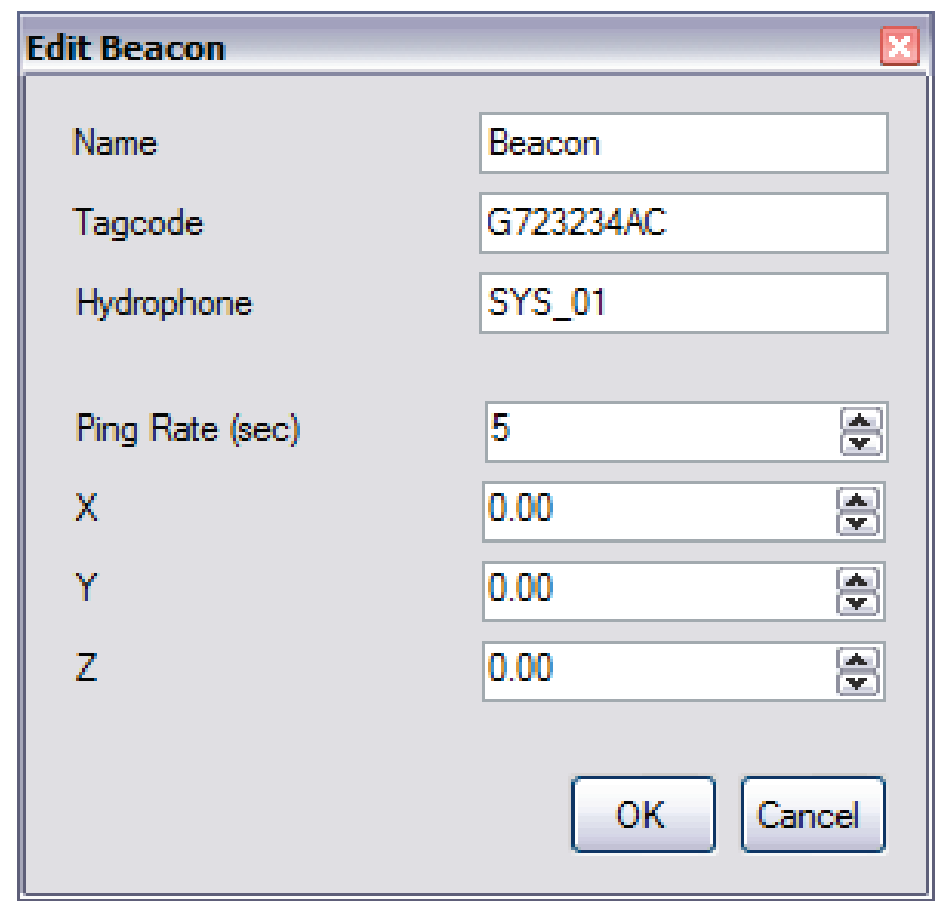

Figure 6.2. Edit Beacon Dialog Box 


\subsection{Deleting a Single Beacon}

To delete a beacon, select the beacon from the list by clicking on the row, which will highlight the row in light gray (Figure 6.3). When the Delete button is selected, the beacon will be removed from the list.

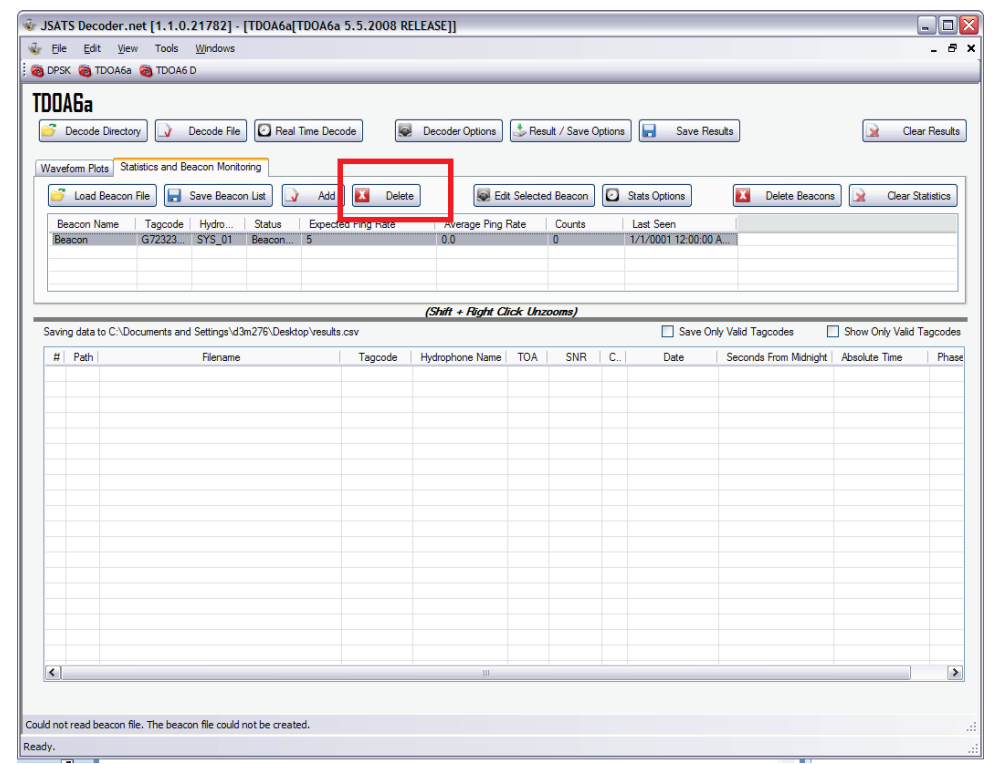

Figure 6.3. To Delete a Beacon, Highlight the Row and Select Delete

\subsection{Deleting All Beacons from the List}

To delete all beacons from the Beacon List, press the Delete Beacons button (Figure 6.1).

When the Delete Beacons button is pressed, an alert dialog box will open (Figure 6.4) requesting confirmation of the command to clear the beacon list. Select Yes to clear all beacons from the list or No to cancel the Delete Beacons command.

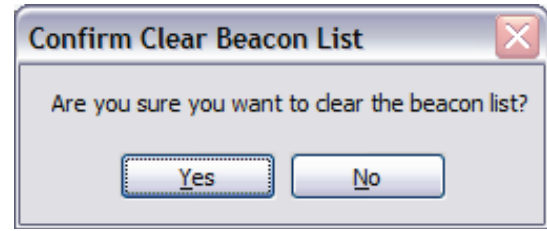

Figure 6.4. The Confirmation Dialog Box to Delete All Beacons on the List

\subsection{Editing a Beacon}

To edit the information for a beacon, select the beacon from the list and press the Edit Selected Beacon button (Figure 6.1). The Edit Beacon dialog box will open (Figure 6.2). Edit the beacon information as needed, then press $\mathbf{O K}$ to accept changes or Cancel to reject the changes. 


\subsection{Loading a Beacon File}

Beacon information can also be entered into the beacon list using an ASCII text file. Each line of the file contains the information for one beacon in the correct file format, as described in Section 6.1. An example text file displaying three beacons as viewed with Notepad is shown in Figure 6.5.

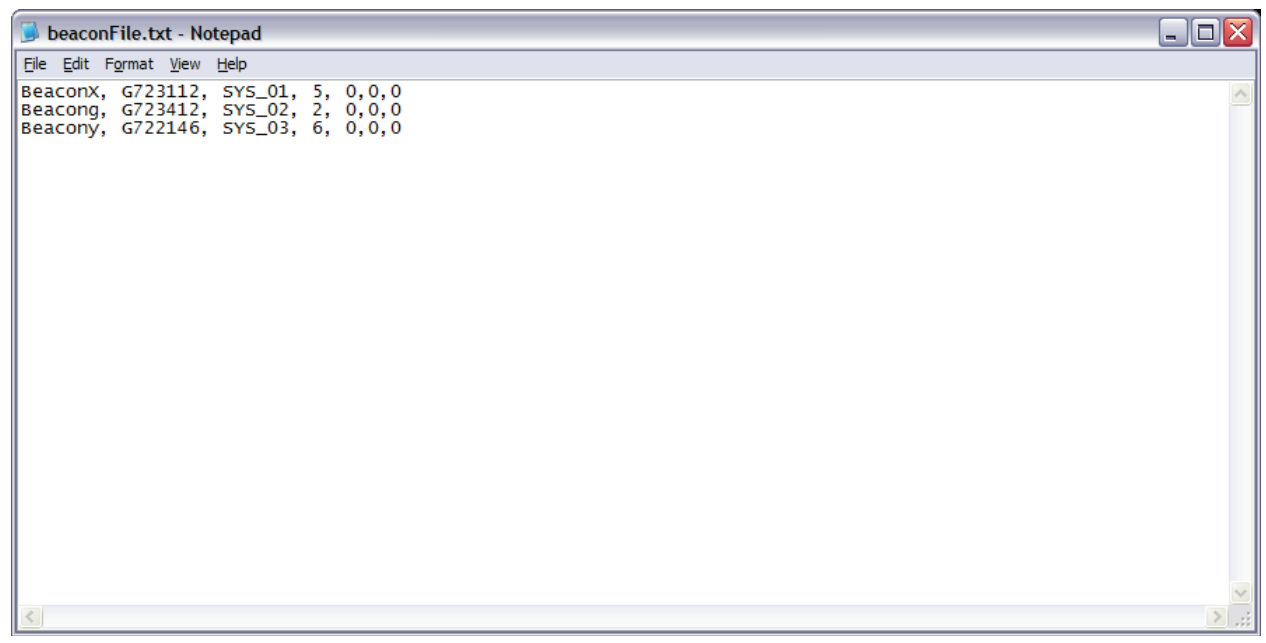

Figure 6.5. Beacon List File Example Using Notepad

The sequence of operations to load a beacon text file is initiated by pressing the Load Beacon File button (Figure 6.1).

Navigate to the beacon text file in the Open file dialog box and initiate it by clicking the Open button (Figure 6.6). The file loads to the spreadsheet as shown in Figure 6.7.

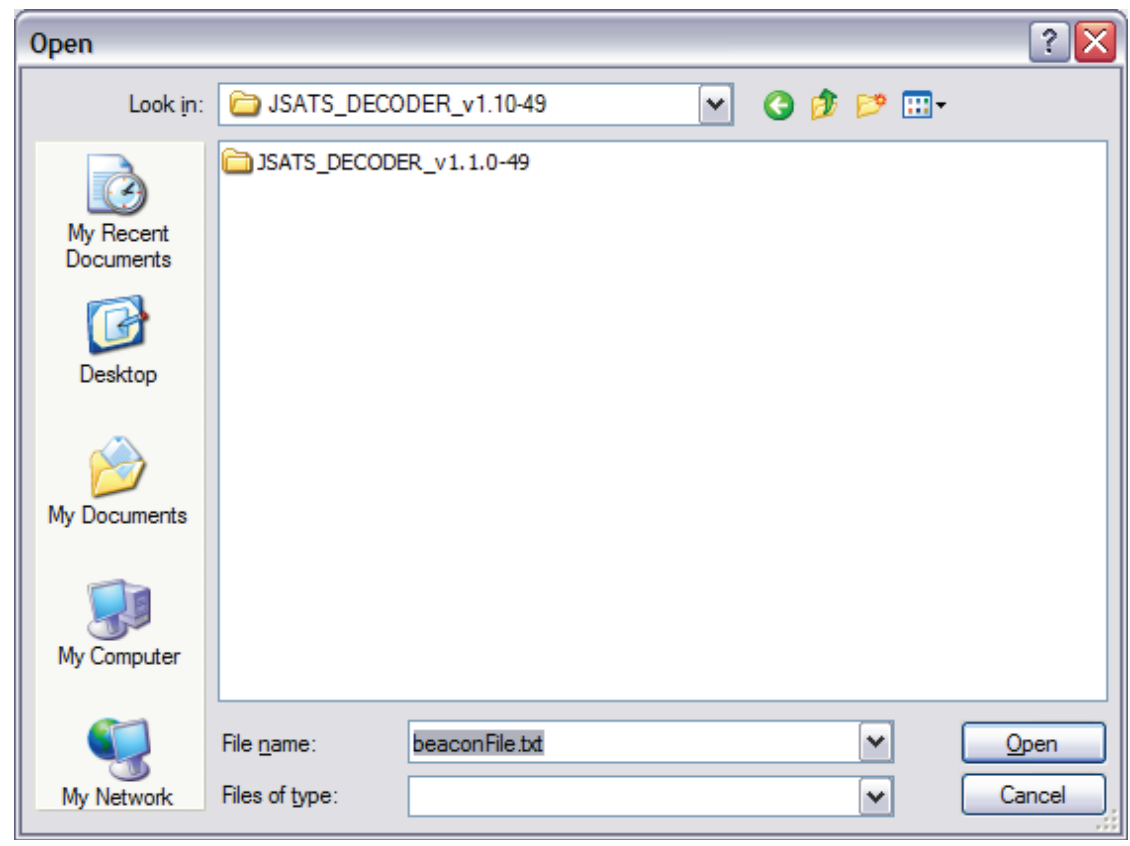

Figure 6.6. The Text File Containing Beacon Information Is Selected from the Open File Dialog Box 


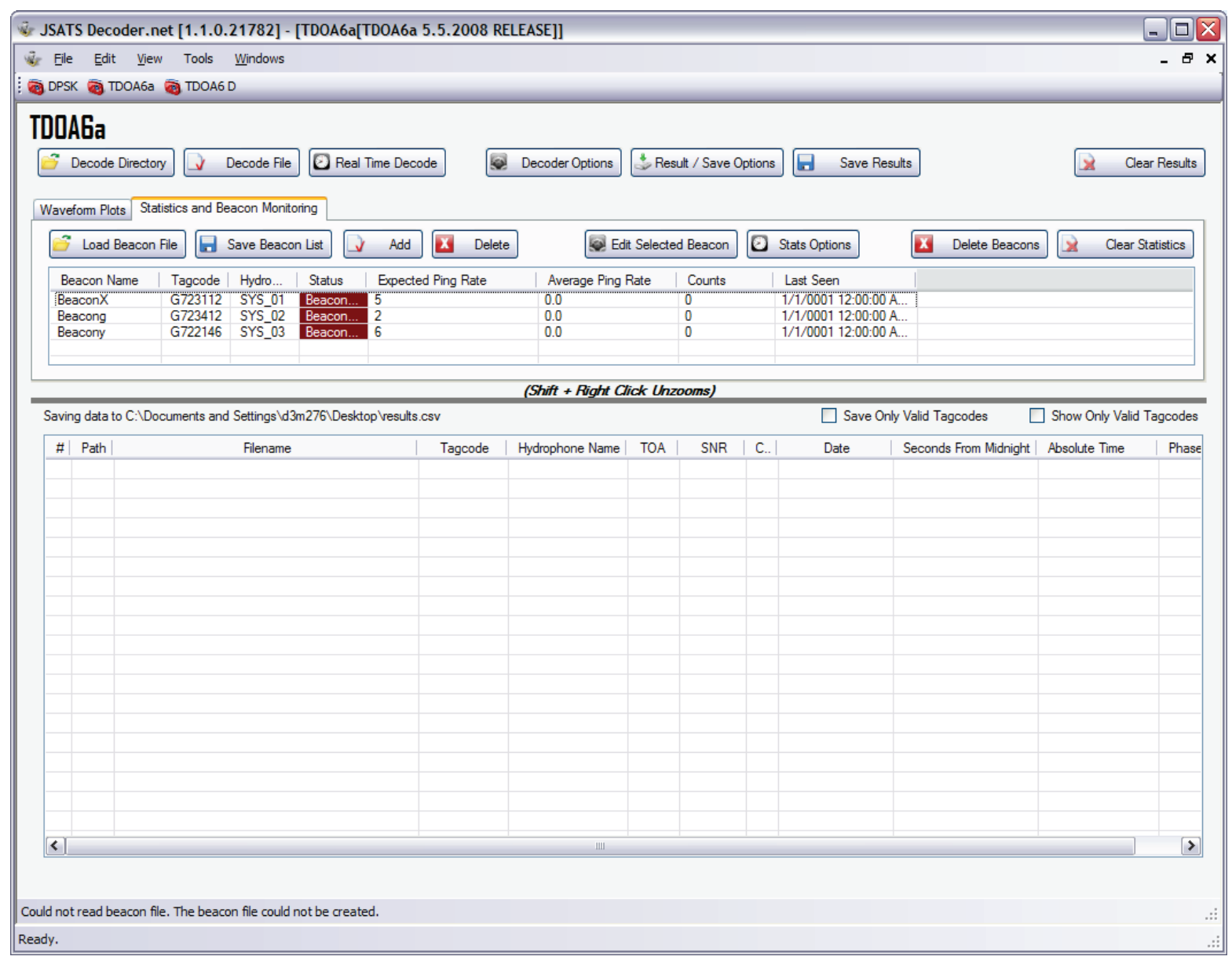

Figure 6.7. Beacon List Loaded from an ASCII Text File

\subsection{Saving a Beacon File}

When a beacon list is complete, it is saved by pressing the Save Beacon List button (Figure 6.1).

When the Save Beacon List button is selected the Save As file dialog box opens (Figure 6.8). Create or navigate to the directory to which to save the beacon list and type the name of the new beacon list file. Select the Save button to save the file or Cancel to reject the process. 


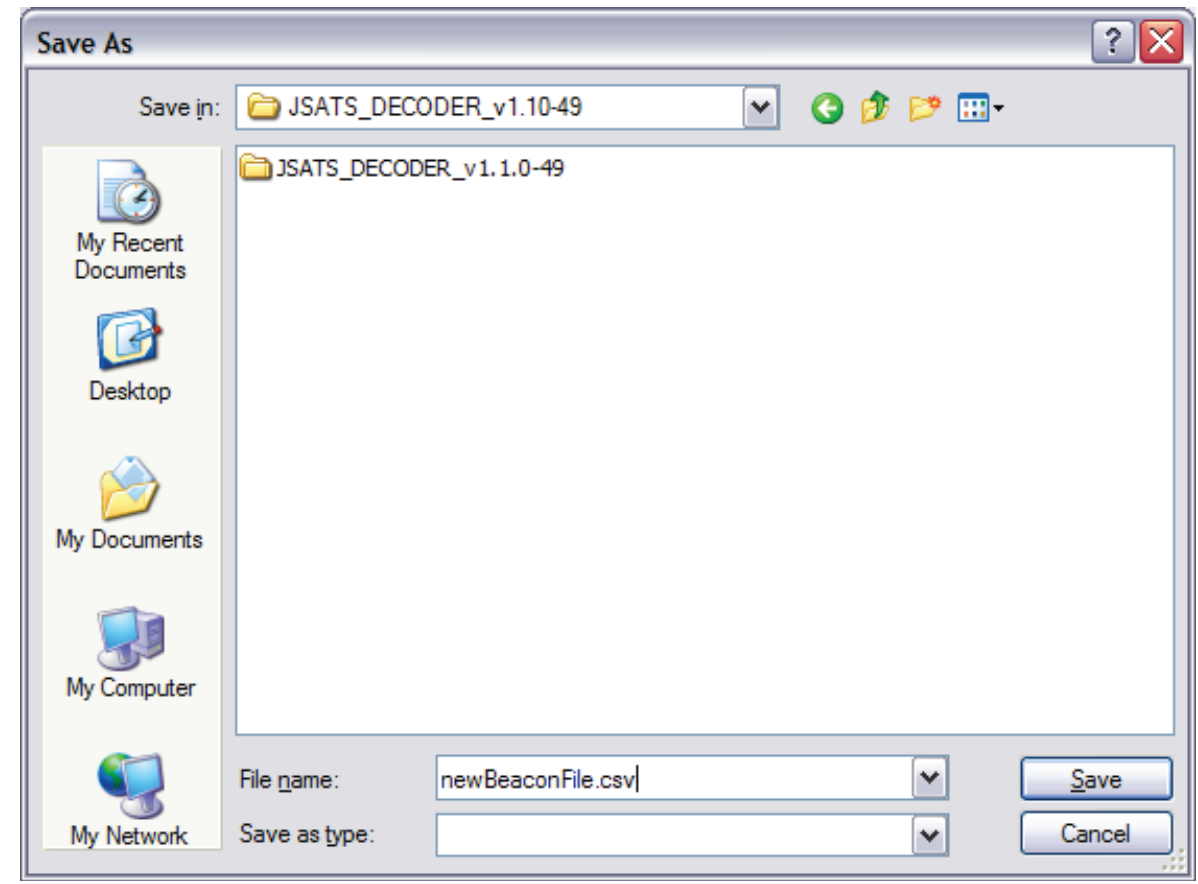

Figure 6.8. When Saving a Beacon List the Save As Dialog Box Opens, Prompting Insertion of a File Name

The decoder keeps a local list of all manually added beacons or those loaded using ASCII text files. The Save Beacon List function saves a beacon list as a file that can be loaded into the decoder program running on other computer systems.

\subsection{Setting Statistics Options and Saving Stats Options}

The details for the computation of beacon statistics can be changed by pressing the Stats Options button (Figure 6.1).

Pressing the Stats Options button opens the Statistics Options dialog box shown in Figure 6.9. 


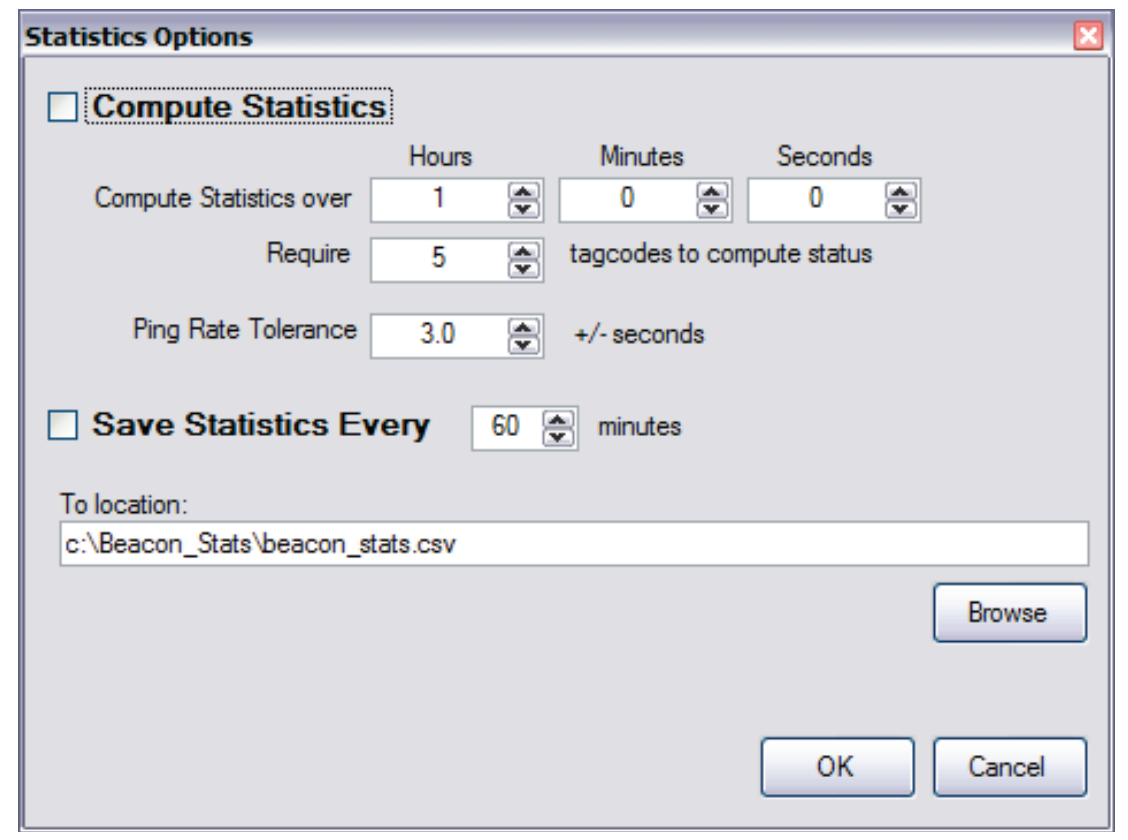

Figure 6.9. Statistics Options Dialog Box

\subsubsection{Compute Statistics}

In the Statistics Options dialog box, the Compute Statistics box must be checked for statistics to be computed for tag codes or beacons (Figure 6.9).

\subsubsection{Time Period for Computing Statistics}

The Compute Statistics Over option identifies the time period in hours, minutes, and seconds within which the decoder will compute statistics. Statistics are calculated over a moving window of the duration specified. By default the duration is an hour, as shown in Figure 6.9.

\subsubsection{Tag Codes Required to Compute Statistics}

The Require [-] Tagcodes to Compute Status option designates the number of observations the decoder will make before computing the statistics needed to report beacon status (Figure 6.9).

\subsubsection{Ping Rate Tolerance}

The average ping rate value will seldom exactly match the expected ping rate value. The Ping Rate Tolerance option allows the user to set a specific range within which the average ping rate can vary and still be considered to be working correctly. The example in Figure 6.9 shows a ping rate tolerance range of $+/-3$ seconds. 


\subsubsection{Saving Statistics}

The Save Statistics Every [-] Minutes checkbox and spin box instruct the decoder how frequently to save statistics to the statistics file described in Section 6.8.6. The Save Statistics Every

[-] Minutes box must be checked to enable the save function (Figure 6.9).

\subsubsection{Saving Beacon Statistics}

The To Location text box identifies the location where the statistics file will be saved. During each save, the new statistics will be appended to the statistics file. Enter the directory and name of the file in the text box or press the Browse button, navigate to the preferred directory, and enter a file name (Figure 6.10). Select Open to save or Cancel to reject the process.

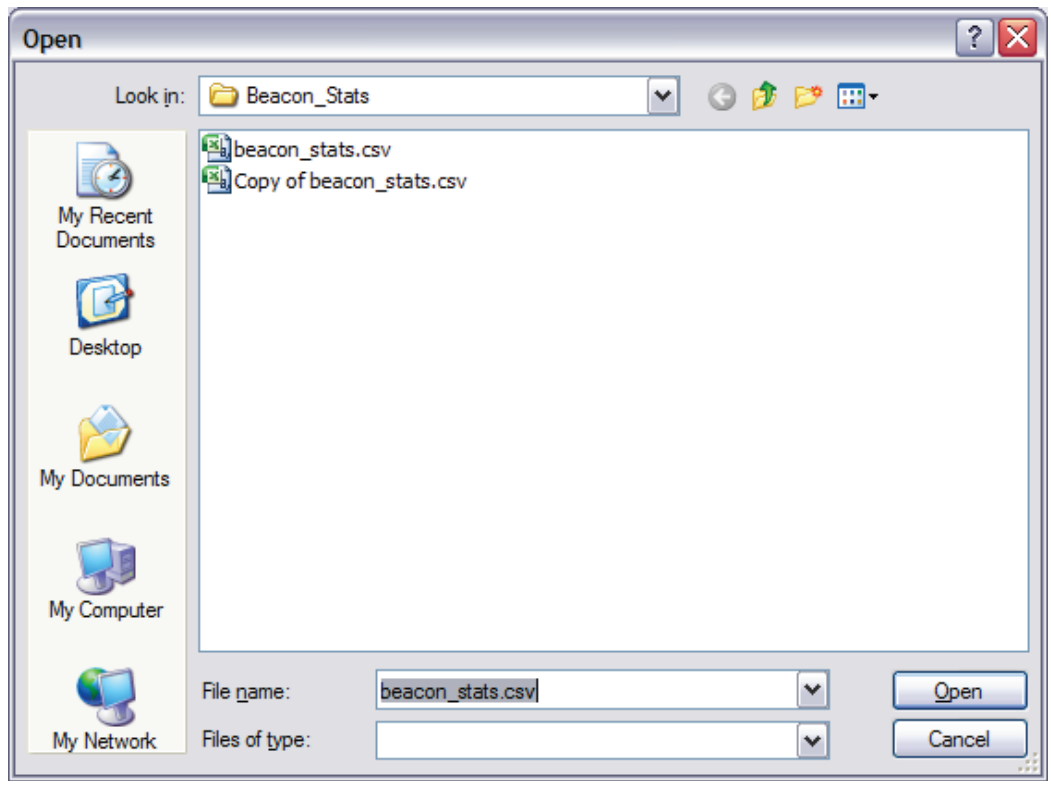

Figure 6.10. The Open Dialog Box where Beacon Statistics Will Be Saved

\subsection{Clearing Statistics}

The beacon statistics being displayed in the Decoder window can be cleared by pressing the Clear Statistics button (Figure 6.1). The results will be deleted and will not be saved unless saved previously. 


\subsection{Data and File Formats}

File formats for beacons, beacon statistics, and decoder results are described in this section.

\subsection{Beacon File Format}

The beacon file configuration is a comma separated file format as shown in Figure 7.1. The columns within a row for a single beacon are as follows:

- Beacon Name

- Beacon Tagcode

- Hydrophone Name

- Expected Ping Rate

- X Position

- Y Position

- Z Position.

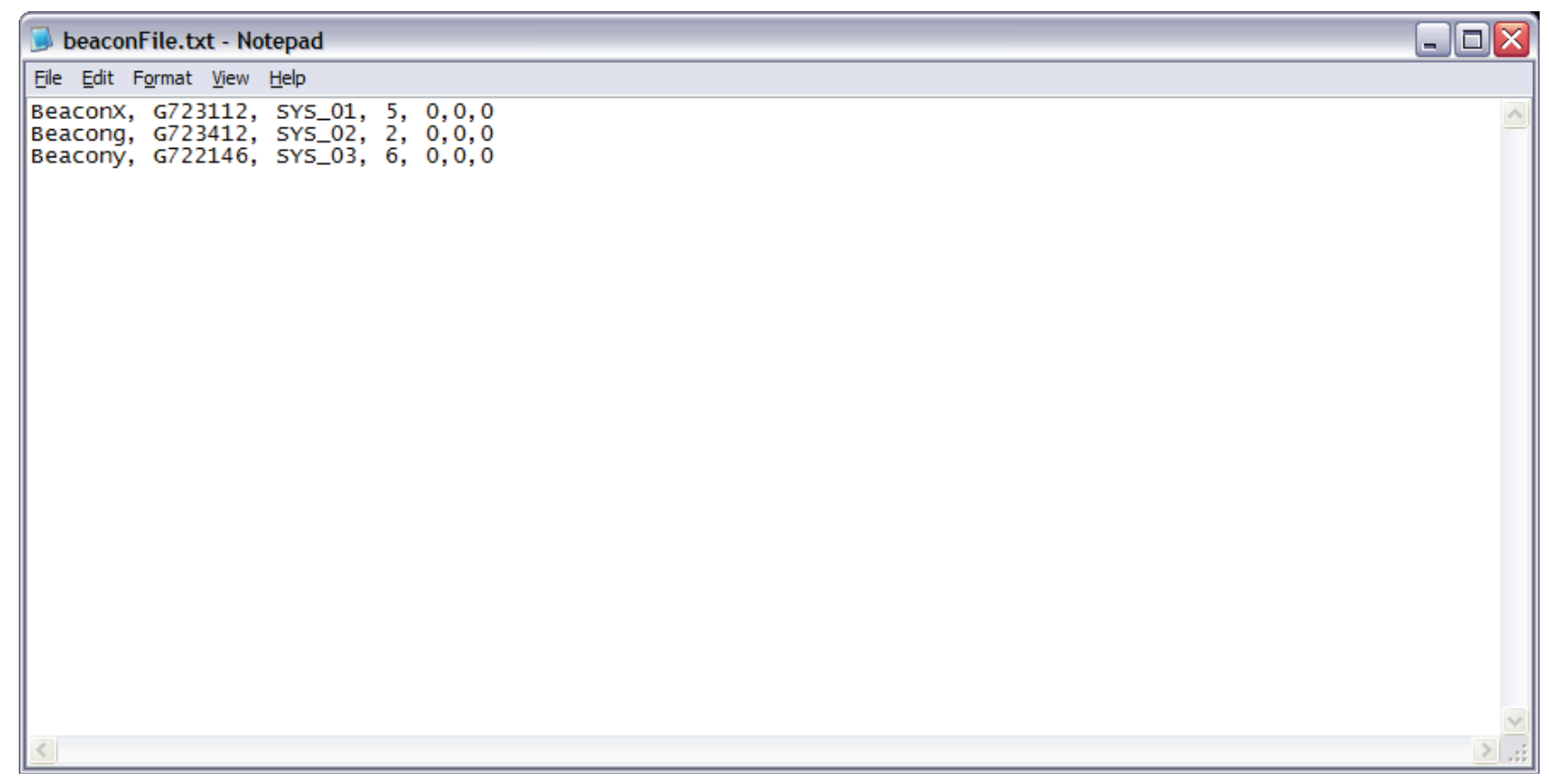

Figure 7.1. Beacon File Format Example

The X, Y, and Z position columns are not used by the decoder and are only placeholders to indicate the location of the beacon if needed for reference purposes. 


\subsection{Beacon Statistics File Format}

The beacon statistics file (Figure 7.2), which is appended after every Save Beacon Statistics procedure, has the following format:

- Statistics Heading - Time and Date of save

- Column Headings:

- Beacon Name

- Beacon Tagcode

- Hydrophone Name

- Expected Ping Rate

- Average (or observed) Ping Rate

- Position X

- Position Y

- Position Z.

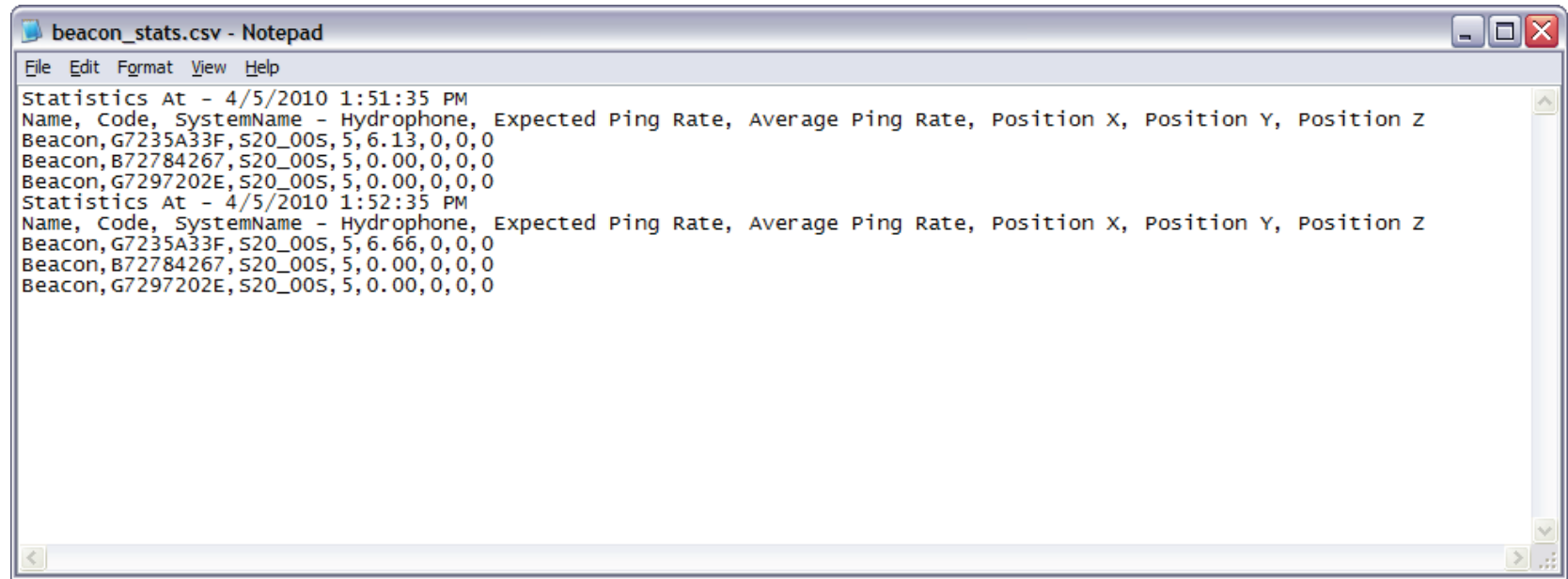

Figure 7.2. Beacon Statistics File Format

Specific time and date statistics and column headings are repeated each time the file is appended.

\subsection{Decoder Results File Format}

The Results file is formatted as shown in the Excel spreadsheet in Figure 7.3. Data rows are configured as follows:

- The first line is blank.

- The second line describes the decoder's assembly file, useful developer information for troubleshooting. 
- The third line describes the decoder type (name), version, and options.

- The fourth line contains the column headings.

- The next lines are the data. Each line represents a single tag code, possibly from the same hydrophone, possibly from the same file.

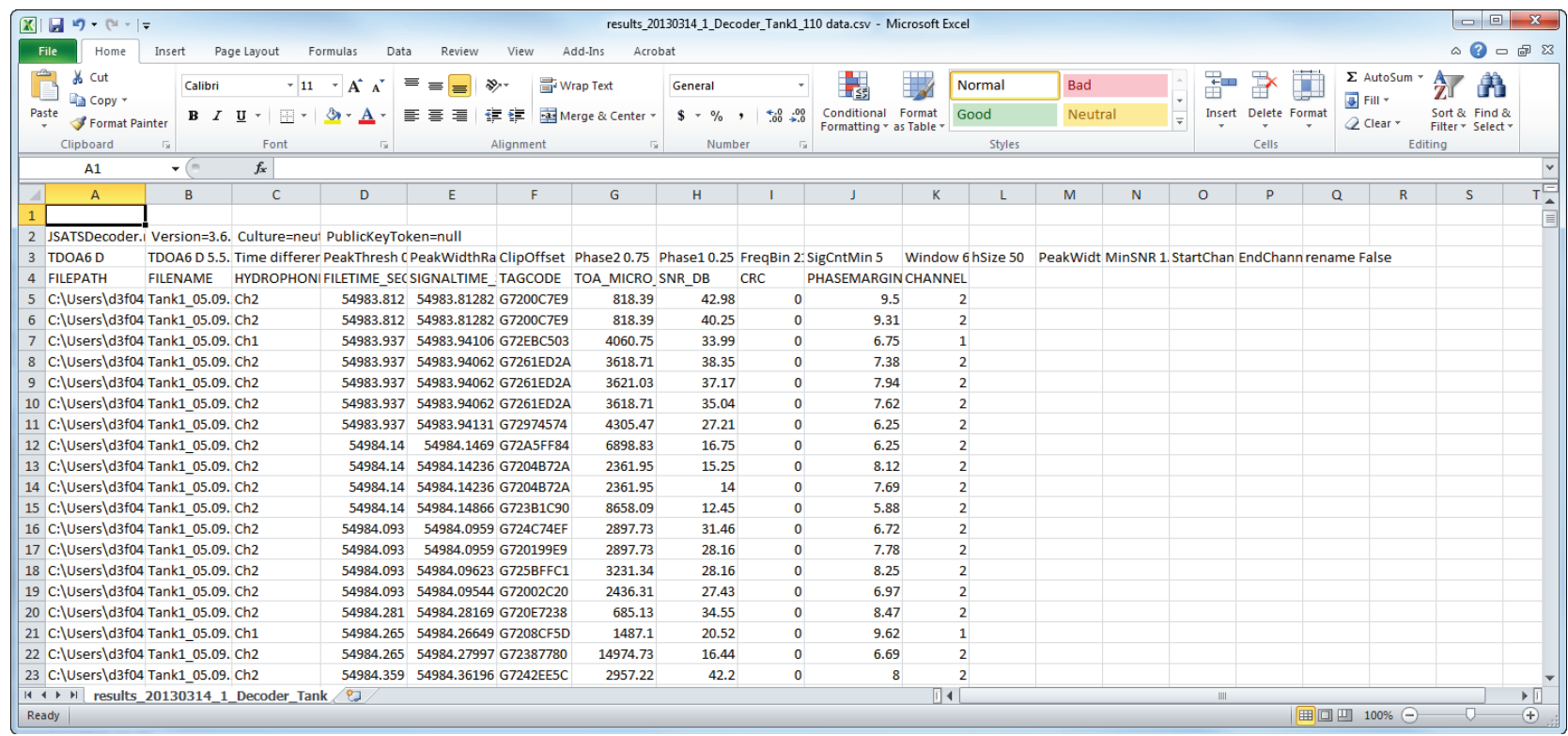

Figure 7.3. Results File Format

The data columns contain the following information:

- FILEPATH - directory name of the decoded waveform file

- FILENAME - file name

- HYDROPHONENAME - name of hydrophone where the tag waveform was detected

- FILETIME_SEC_FROMMIDNIGHT - seconds from midnight (fractional) when the file was created using GPS clock

- SIGNALTIME_SEC_FROMMIDNIGHT - seconds from midnight (fractional) when the tag signal was detected using the JSATS receiver GPS clock

- TAGCODE - tag code decoded from the detected waveform

- TOA_MICRO_SEC - time of arrival relative to the start of the file in microseconds signal when the waveform was decoded

- SNR_DB - Signal-to-noise ratio in decibels - SNR for the waveform relative to the background noise of the environment

- $\mathrm{CRC}$ - cyclic redundancy check computed over the 16-bit binary representation of the tag code

- PHASEMARGIN - confidence metric for binary phase-shift keying phase transitions

- CHANNEL - data channel. 



\subsection{Command Line Decoder}

The Command Line decoder is a lightweight version of the JSATS decoder. This version is intended for post-processing of . $\mathrm{bwm}$ files and is not designed for real-time decoding. All major post-processing functionality in the graphical user interface (GUI) version of the JSATS decoder is available in the Command Line decoder.

The argument format for the Command Line decoder is as follows:

JSATSDecoderCommandline.exe $<$ directory containing . bwm files $>$ [-r to check subdirectories] [- o .csv file name] [-p to use parallel processing].

The path to decode is required; the options (parallel, out file, and parallel) are not. By default, only top-level directories are searched, waveform files are decoded serially, and output is written to results.csv files and will be in the same directory as the program.

For example, to decode all waveform files in the directory $C: \mid . b w m$ Files, the following command is entered:

$$
\text { > JSATSDecoderCommandLine.exe "C:I.bwm Files" }
$$

Decoding speed can be increased using parallel decoding. To enable parallel decoding, the following command is entered:

$$
\text { >JSATSDecoderCommandLine.exe "C:ı.bwm Files"-p }
$$

If the results are to be saved to $C: \mid m y$ Results.csv and decoded in parallel, the following command is entered:

$$
\text { > JSATSDecoderCommandLine.exe "C:|.bwm Files" - p -o C:|myResults.csv }
$$

To check all the files in the $C: 1 . b w m$ Files sub-directories, the -r option is used:

$$
\text { >JSATSDecoderCommandLine.exe "C:|.bwm Files" }-r
$$

For example, two hard drives labeled E: and F:, respectively, contain tag waveforms to be decoded. Decoding needs to be completed as quickly as possible, so the decoders will be run on the processing computer and results will be saved on the computer desktop.

Two instances of the Command Line decoder prompts are needed to decode the waveform files on the hard drives. The first prompt is

>JSATSDecoderCommandLine.exe "E:|" $-p-r-o$ "C:|Users $\mid$ User|Desktople drive.csv"

After entering the first prompt, press Enter and select the second prompt. The second prompt will be:

$$
\text { >JSATSDecoderCommandLine.exe " } F: \mid \text { " }-p-r-o \text { "C:|Users } \mid \text { User } \mid \text { Desktop } \mid f \text { drive.csv" }
$$


After the prompt has been completed, press Enter to begin processing the waveform files. The format of the results files from the Command Line decoder will be of the same format as the GUI version of the JSATS decoder (see Figure 7.3). 


\subsection{References}

Deng Z, MA Weiland, T Fu, TA Seim, BL Lamarche, EY Choi, TJ Carlson, and MB Eppard. 2011. "A Cabled Acoustic Telemetry System for Detecting and Tracking Juvenile Salmon: Part 2. ThreeDimensional Tracking and Passage Outcomes.” Sensors 11(6):5661-5676.

McMichael GA, MB Eppard, TJ Carlson, JA Carter, BD Ebberts, RS Brown, MA Weiland, GR Ploskey, RA Harnish, and Z Deng. 2010. “The Juvenile Salmon Acoustic Telemetry System; A New Tool." Fisheries 35(1):9-22.

Weiland MA, Z Deng, TA Seim, BL Lamarche, EY Choi, T Fu, TJ Carlson, AI Thronas, and MB Eppard. 2011. "A cabled acoustic telemetry system for detecting and tracking juvenile salmon: Part 1.

Engineering design and instrumentation." Sensors 11(6):5645-5660. 




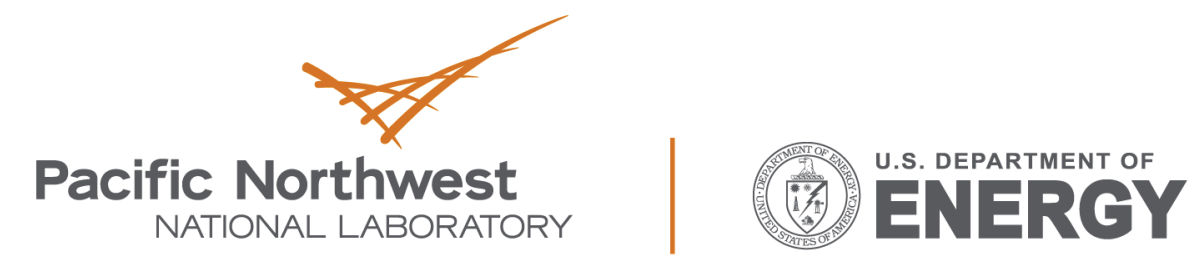

Proudly Operated by Battelle Since 1965

902 Battelle Boulevard

P.O. Box 999

Richland, WA 99352

1-888-375-PNNL (7665)

www.pnnl.gov 